\title{
GLOBAL ESTIMATES FOR THE FUNDAMENTAL SOLUTION OF HOMOGENEOUS HÖRMANDER OPERATORS
}

\author{
STEFANO BIAGI, ANDREA BONFIGLIOLI, AND MARCO BRAMANTI
}

\begin{abstract}
Let $\mathcal{L}=\sum_{j=1}^{m} X_{j}^{2}$ be a Hörmander sum of squares of vector fields in $\mathbb{R}^{n}$, where any $X_{j}$ is homogeneous of degree 1 with respect to a family of non-isotropic dilations in $\mathbb{R}^{n}$. Then $\mathcal{L}$ is known to admit a global fundamental solution $\Gamma(x ; y)$, that can be represented as the integral of a fundamental solution of a sublaplacian operator on a lifting space $\mathbb{R}^{n} \times \mathbb{R}^{p}$, equipped with a Carnot group structure. The aim of this paper is to prove global pointwise (upper and lower) estimates of $\Gamma$, in terms of the Carnot-Carathéodory distance induced by $X=\left\{X_{1}, \ldots, X_{m}\right\}$ on $\mathbb{R}^{n}$, as well as global pointwise (upper) estimates for the $X$-derivatives of any order of $\Gamma$, together with suitable integral representations of these derivatives. The least dimensional case $n=2$ presents several peculiarities which are also investigated. Applications to the potential theory for $\mathcal{L}$ and to singular-integral estimates for the kernel $X_{i} X_{j} \Gamma$ are also provided. Finally, most of the results about $\Gamma$ are extended to the case of Hörmander operators with drift $\sum_{j=1}^{m} X_{j}^{2}+X_{0}$, where $X_{0}$ is 2-homogeneous and $X_{1}, \ldots, X_{m}$ are 1-homogeneous.
\end{abstract}

Mathematics Subject Classification: 35A08, 35C15, 35B45 (primary), 35J70, 35H10, 26D10 (secondary).

Keywords: Fundamental solution; Global a priori estimates; Homogeneous Hörmander operators; CarnotCarathéodory spaces; Integral representation of solutions.

\section{INTRODUCTION AND MAIN RESULTS}

In this paper we consider a class of linear second order partial differential operators

$$
\mathcal{L}=X_{1}^{2}+\cdots+X_{m}^{2},
$$

where $X=\left\{X_{1}, \ldots, X_{m}\right\}$ is a set of Hörmander vector fields in $\mathbb{R}^{n}(n \geq 2)$, and any element of $X$ is homogeneous of degree 1 with respect to a family $\left\{\delta_{\lambda}\right\}_{\lambda>0}$ of non-isotropic dilations. (Precise definitions will be given below). Our main aim is to prove global pointwise (upper and lower) estimates, in terms of the Carnot-Carathéodory distance $d_{X}$ induced by $X$ on $\mathbb{R}^{n}$, of a suitable positive global fundamental solution $\Gamma(x ; y)$ for $\mathcal{L}$, and global pointwise (upper) estimates for the $X$-derivatives of any order of $\Gamma$ (see Theorem 1.3). The least dimensional case $n=2$ seems to be particularly delicate, as fundamental solutions near the diagonal may exhibit different behavior from point to point (logarithmic or power-like). Furthermore, applications to the potential theory for $\mathcal{L}$ (Section 7) and to singular integral estimates for the kernel $X_{i} X_{j} \Gamma$ (Section 8) are provided.

Most profound ideas in the study of the geometrical subelliptic analysis of general Hörmander sums of squares of vector fields $L=\sum_{j=1}^{m} Y_{j}^{2}$ (of which our $\mathcal{L}$ is a particular case) are contained in the seminal papers by Hörmander [24], by Folland [19], by Rothschild and Stein [28, by Nagel, Stein and Wainger [27, by Sánchez-Calle 29. A paramount tool in the analysis of $L$ is RothschildStein's lifting technique, which locally approximates $L$ with a sublaplacian operator on some higher dimensional free Carnot group.

Broadly speaking, in the cited papers, a large part of the most relevant theory for $L$ (geometric analysis, function spaces, subelliptic estimates, etc.) was ultimately settled under its local form. On the other hand, a global theory (to which we are interested) is developed by Folland [19] in the special case of homogeneous left invariant Hörmander operators on homogeneous groups, but is inevitably missing in the general case of $L$, since one cannot expect that $L$ be equipped with a global fundamental solution $\Gamma$ defined out of the diagonal of $\mathbb{R}^{n} \times \mathbb{R}^{n}$, without further assumptions on $L$. Analogously, for such general operators $L$, geometrically meaningful results involving CC-balls $B_{X}(x, r)$ are mainly available when the radius $r$ is sufficiently small and the center $x$ is located in some fixed compact set. The locality of Rothschild-Stein's lifting technique is also an implicit (hardly avoidable) obstruction to a global theory for $L$. 
Hence, if we aim to give global estimates for a globally defined fundamental solution, some further assumptions on the operator must be made. Roughly put, the homogeneity of $L$ with respect to a family of dilations as in (1.1) can help recovering large $r$ 's, whereas the invariance of $L$ with respect to a family of Lie-group translations is of aid in dealing with arbitrary $x$ 's. As is well known, a simultaneous homogeneity/translation-invariance boils down to the case when $L$ is a sublaplacian operator on a Carnot group $\mathbb{G}$, for which a global fundamental solution $\Gamma_{\mathbb{G}}$ is known to exist after Folland's paper [19, and the CC-ball $B_{X}(x, r)$ is just the left translation by $x$ of the $\delta_{r}$-dilated of the ball $B_{X}(0,1)$, so that the underlying subelliptic geometry is much simpler. One can easily say that the worthwhile results of the subelliptic analysis for the sublaplacians in the Carnot group case are nowadays well established.

A convenient framework, more general than the Carnot group setting, is the one considered in this paper, that is the case when $\mathcal{L}$ is $\delta_{\lambda}$-homogeneous of degree 2 , but not left-invariant.

Example 1.1. (1). A first instance of such operators are the Grushin-type PDO's in $\mathbb{R}^{2}$

$$
\left(\partial_{x_{1}}\right)^{2}+\left(x_{1}^{k} \partial_{x_{2}}\right)^{2} \quad(\text { with } k \in \mathbb{N}),
$$

associated with the dilations $\left(\lambda x_{1}, \lambda^{k+1} x_{2}\right)$. These are not left-invariant PDO's on any Lie group on $\mathbb{R}^{2}$ (when $k \geq 1$ ) as $x_{1}^{k} \partial_{x_{2}}$ vanishes when $x_{1}=0$ without being the null vector field.

(2). Another class of operators to which our theory applies is given by

$$
\left(\partial_{x_{1}}\right)^{2}+\left(x_{1} \partial_{x_{2}}+x_{2} \partial_{x_{3}}+\ldots+x_{n-1} \partial_{x_{n}}\right)^{2} \text { in } \mathbb{R}^{n}
$$

which is $\delta_{\lambda}$-homogeneous of degree 2 (but not left invariant on $\mathbb{R}^{n}$, for the same reasons as in (1)) with respect to the dilations $\delta_{\lambda}(x)=\left(\lambda x_{1}, \lambda^{2} x_{2}, \cdots, \lambda^{n} x_{n}\right)$.

(3). A further example is the operator

$$
X_{1}^{2}+X_{2}^{2}=\left(\partial_{x_{1}}\right)^{2}+\left(x_{1} \partial_{x_{2}}+x_{1}^{2} \partial_{x_{3}}\right)^{2} \text { on } \mathbb{R}^{3}
$$

which is homogeneous of degree 2 (but not left invariant on $\mathbb{R}^{3}$ ) with respect to

$$
\delta_{\lambda}(x)=\left(\lambda x_{1}, \lambda^{2} x_{2}, \lambda^{3} x_{3}\right) .
$$

The Lie algebra generated by $X_{1}, X_{2}$ is the Lie algebra of the so-called Engel group on $\mathbb{R}^{4}$.

(4). Finally, the operator

$$
\left(\partial_{x_{1}}\right)^{2}+\left(x_{1} \partial_{x_{2}}+x_{1}^{2} \partial_{x_{3}}+\cdots+x_{1}^{n-1} \partial_{x_{n}}\right)^{2} \text { on } \mathbb{R}^{n}
$$

is homogeneous of degree 2 with respect to the same dilations as in (2), but not left invariant on $\mathbb{R}^{n}$.

Let us now precisely fix our assumptions. We assume that $X=\left\{X_{1}, \ldots, X_{m}\right\}$ fulfils the following conditions (H.1), (H.2), (H.3):

(H.1) there exists a family of (non-isotropic) dilations $\left\{\delta_{\lambda}\right\}_{\lambda>0}$ of the form

$$
\delta_{\lambda}: \mathbb{R}^{n} \longrightarrow \mathbb{R}^{n} \quad \delta_{\lambda}(x)=\left(\lambda^{\sigma_{1}} x_{1}, \ldots, \lambda^{\sigma_{n}} x_{n}\right),
$$

where $1=\sigma_{1} \leq \cdots \leq \sigma_{n}$, such that $X_{1}, \ldots, X_{m}$ are $\delta_{\lambda}$-homogeneous of degree 1 , i.e.,

$$
X_{j}\left(f \circ \delta_{\lambda}\right)=\lambda\left(X_{j} f\right) \circ \delta_{\lambda}, \quad \text { for every } \lambda>0, f \in C^{\infty}\left(\mathbb{R}^{n}\right) \text { and } j=1, \ldots, m \text {. }
$$

In what follows, we denote by

$$
q:=\sum_{j=1}^{m} \sigma_{j}
$$

the so-called $\delta_{\lambda}$-homogeneous dimension of $\left(\mathbb{R}^{n}, \delta_{\lambda}\right)$.

(H.2) $X_{1}, \ldots, X_{m}$ are linearly independent] and satisfy Hörmander's rank condition at 0, i.e.,

$$
\operatorname{dim}\{Y(0): Y \in \operatorname{Lie}(X)\}=n .
$$

\footnotetext{
${ }^{1}$ The linear independence of the $X_{i}$ 's is meant with respect to the vector space of the smooth vector fields on $\mathbb{R}^{n}$; this must not be confused with the linear independence of the vectors $X_{1}(x), \ldots, X_{m}(x)$ in $\mathbb{R}^{n}$ (when $\left.x \in \mathbb{R}^{n}\right)$ : the latter is sufficient but not necessary to the former linear independence. Thus, $X_{1}=\partial_{x_{1}}$ and $X_{2}=x_{1} \partial_{x_{2}}$ are linearly independent vector fields, even if $X_{1}\left(0, x_{2}\right) \equiv(1,0)$ and $X_{2}\left(0, x_{2}\right) \equiv(0,0)$ are dependent vectors of $\mathbb{R}^{2}$.
} 
Here Lie $(X)$ stands for the smallest Lie subalgebra of $X\left(\mathbb{R}^{n}\right)$ containing $X$, where $X\left(\mathbb{R}^{n}\right)$ is the Lie algebra of all the smooth vector fields on $\mathbb{R}^{n}$.

Finally, we make the following dimensional assumptions:

(H.3) we require that $q>2$ and

$$
N:=\operatorname{dim}(\operatorname{Lie}\{X\})>n \geq 2 .
$$

Throughout the paper we let $p:=N-n \geq 1$ and we denote the points of $\mathbb{R}^{N} \equiv \mathbb{R}^{n} \times \mathbb{R}^{p}$ by

$$
(x, \xi), \quad \text { with } x \in \mathbb{R}^{n} \text { and } \xi \in \mathbb{R}^{p} \text {. }
$$

Remark 1.2 (Some consequences of (H.1)-to-(H.3)). Assumptions (H.1) and (H.2) together imply that the $\sigma_{i}$ 's in (1.1) are integers, and that $\operatorname{Lie}\{X\}$ is nilpotent of step $\sigma_{n}$ (see e.g., 10]).

We observe that, by (H.1), the validity of Hörmander's rank condition at 0 (condition (H2)) implies its validity at any other point $x \in \mathbb{R}^{n}$ (this is proved in Remark 3.2). Thus, the Hörmander operator $\mathcal{L}=\sum_{j=1}^{m} X_{j}^{2}$ is $C^{\infty}$-hypoelliptic on every open subset of $\mathbb{R}^{n}$.

The assumption $q>2$ in (H3) is harmless since the case $q=2$ only happens when $\mathcal{L}$ is a strictly-elliptic constant-coefficient operator in $\mathbb{R}^{2}$ (which is also left invariant on $\left(\mathbb{R}^{2},+\right)$ ), a wellknown situation we are not interested in. The assumption $N>n$ in (H3) is rather harmless as well, for the following reason. Since $X$ is a Hörmander set, $N$ defined in (1.3) cannot be $<n$; on the other hand, when $N=n$, it follows from a general result contained in [9] (and exploiting the $\delta_{\lambda}$-homogeneity of the elements of $X$ ) that $\mathcal{L}$ is necessarily a sublaplacian on a homogeneous Carnot group on $\mathbb{R}^{n}$, a well-studied situation in which the results of this paper are already known (see [19]).

Very recently, under assumption (1.3), the existence of a global fundamental solution $\Gamma$ for $\delta_{\lambda}$-homogenous $\mathcal{L}$ 's has been obtained in [10] via a lifting procedure due to Folland [20], a global simplified version of Rothschild-Stein's lifting. Folland's technique consists in lifting $\mathcal{L}$ directly to a sublaplacian $\mathcal{L}_{\mathbb{G}}$ on a (strictly higher dimensional) Carnot group ( $\mathbb{G}, *$ ) (which is not necessarily free). After an appropriate change of variable (performed in [10]), one can suppose that the manifold of $\mathbb{G}$ takes the product form $\mathbb{G}=\mathbb{R}_{x}^{n} \times \mathbb{R}_{\xi}^{p}$, with $p=N-n$. Under assumption (1.3), this $p$ is at least 1 . We are now going to review this result, which also gives an integral representation for $\Gamma$; this representation will be used throughout the paper.

In what follows, we refer to [13, §1.4] for the notions of sublaplacian and of homogeneous Carnot group, with the sole difference that we do not require the exponents of the associated dilations $D_{\lambda}$ to be increasingly ordered; this is because we have already performed a change of variable on $\mathbb{R}^{N} \equiv \mathbb{R}^{n} \times \mathbb{R}^{p}$, which separates the unlifted variables $x$ from the lifting variables $\xi$. We also implicitly invoke Folland's result [19] on the existence of a global fundamental solution for any sublaplacian on any Carnot group.

Theorem A ([10, Theorems 3.2 and 4.4]). Assume that $X=\left\{X_{1}, \ldots, X_{m}\right\}$ satisfies (H.1)-to-(H.3), of which we inherit the notation. Then the following facts hold:

(1). There exist a homogeneous Carnot group $\mathbb{G}=\left(\mathbb{R}^{N}, *, D_{\lambda}\right)$ of homogeneous dimension $Q>q$ and a system $\left\{\widetilde{X}_{1}, \ldots, \widetilde{X}_{m}\right\}$ of Lie-generators of Lie(G) such that $\widetilde{X}_{i}$ is a lifting of $X_{i}$ for every $i=1, \ldots, m$; by this we mean that

$$
\widetilde{X}_{i}(x, \xi)=X_{i}(x)+R_{i}(x, \xi)
$$

where $R_{i}(x, \xi)$ is a smooth vector field operating only in the variable $\xi \in \mathbb{R}^{p}$, with coefficients possibly depending on $(x, \xi)$. In particular, the $\widetilde{X}_{i}$ 's are $D_{\lambda}$-homogeneous of degree 1 .

(2). If $\widetilde{\Gamma}$ is the (unique) smooth fundamental solution of $\sum_{i=1}^{m} \widetilde{X}_{i}^{2}$ vanishing at infinity constructed in [19], then $\mathcal{L}$ admits a global fundamental solution $\Gamma(x ; y)$ under the form

$$
\Gamma(x ; y):=\int_{\mathbb{R}^{p}} \widetilde{\Gamma}((x, 0) ;(y, \eta)) \mathrm{d} \eta \quad\left(\text { for } x \neq y \text { in } \mathbb{R}^{n}\right) .
$$


By saying that $\Gamma$ is a global fundamental solution of $\mathcal{L}$ we mean that the map $y \mapsto \Gamma(x ; y)$ is locally integrable on $\mathbb{R}^{n}$ and that

$$
\int_{\mathbb{R}^{n}} \Gamma(x ; y) \mathcal{L} \varphi(y) \mathrm{d} y=-\varphi(x) \quad \text { for every } \varphi \in C_{0}^{\infty}\left(\mathbb{R}^{n}\right) \text { and every } x \in \mathbb{R}^{n} .
$$

Furthermore, setting $\Gamma_{\mathbb{G}}(\cdot):=\widetilde{\Gamma}(0 ; \cdot)$, the integrand in (1.5) takes the convolution form

$$
\widetilde{\Gamma}((x, 0) ;(y, \eta))=\Gamma_{\mathbb{G}}\left((x, 0)^{-1} *(y, \eta)\right),
$$

valid for any $x \neq y$, so that (1.5) becomes

$$
\Gamma(x ; y)=\int_{\mathbb{R}^{p}} \Gamma_{\mathbb{G}}\left((x, 0)^{-1} *(y, \eta)\right) \mathrm{d} \eta \quad\left(\text { for } x \neq y \text { in } \mathbb{R}^{n}\right) .
$$

(3). $\Gamma$ enjoys further properties: it is smooth out of the diagonal; it is symmetric in $x, y$; it is strictly positive; it is locally integrable on $\mathbb{R}^{n} \times \mathbb{R}^{n}$; it vanishes when $x$ or y go to infinity; it is jointly homogeneous of degree $2-q<0$, i.e.,

$$
\Gamma\left(\delta_{\lambda}(x) ; \delta_{\lambda}(y)\right)=\lambda^{2-q} \Gamma(x, y), \quad x \neq y, \lambda>0 .
$$

Once we have uniquely defined the global fundamental solution $\Gamma$ for $\mathcal{L}$ we are interested in estimating, our main results are contained in the following Theorem 1.3, collecting the content of Theorems 4.1, 5.1, 6.1, 6.3, 6.5 and Lemma 4.3 of the paper. Here and throughout, by 'structural constant' we mean a constant only depending on the objects introduced in the axioms (H.1)-to-(H.3) (like $X$, the $\sigma_{i}$ 's, $q, n, N$, etc.) or other fixed parameters (usually explicitly declared).

Theorem 1.3. Let $\mathcal{L}=\sum_{j=1}^{m} X_{j}^{2}$ satisfy assumptions $(\mathrm{H} 1)-(\mathrm{H} 2)-(\mathrm{H} 3)$, and let $\Gamma$ and $\Gamma_{\mathbb{G}}$ be as in Theorem A. Then the following facts hold.

(I). For any $s, t \geq 1$, and any choice of $i_{1}, \ldots, i_{s}, j_{1}, \ldots, j_{t} \in\{1, \ldots, m\}$, we have the following representation formulas for the $X$-derivatives of $\Gamma$ (holding true for $x \neq y$ in $\mathbb{R}^{n}$ ):

$$
\begin{aligned}
& X_{i_{1}}^{y} \cdots X_{i_{s}}^{y}(\Gamma(x ; \cdot))(y)=\int_{\mathbb{R}^{p}}\left(\widetilde{X}_{i_{1}} \cdots \widetilde{X}_{i_{s}} \Gamma_{\mathbb{G}}\right)\left((x, 0)^{-1} *(y, \eta)\right) \mathrm{d} \eta \\
& X_{j_{1}}^{x} \cdots X_{j_{t}}^{x}(\Gamma(\cdot ; y))(x)=\int_{\mathbb{R}^{p}}\left(\widetilde{X}_{j_{1}} \cdots \widetilde{X}_{j_{t}} \Gamma_{\mathbb{G}}\right)\left((y, 0)^{-1} *(x, \eta)\right) \mathrm{d} \eta \\
& X_{j_{1}}^{x} \cdots X_{j_{t}}^{x} X_{i_{1}}^{y} \cdots X_{i_{s}}^{y} \Gamma(x ; y) \\
& \quad=\int_{\mathbb{R}^{p}}\left(\widetilde{X}_{j_{1}} \cdots \widetilde{X}_{j_{t}}\left(\left(\widetilde{X}_{i_{1}} \cdots \widetilde{X}_{i_{s}} \Gamma_{\mathbb{G}}\right) \circ \iota\right)\right)\left((y, 0)^{-1} *(x, \eta)\right) \mathrm{d} \eta .
\end{aligned}
$$

Here $\iota$ denotes the inversion map of the Lie group $\mathbb{G}$.

(II). For any integer $r \geq 1$ there exists $C_{r}>0$ such that

$$
\left|Z_{1} \cdots Z_{r} \Gamma(x ; y)\right| \leq C_{r} \frac{d_{X}(x, y)^{2-r}}{\left|B_{X}\left(x, d_{X}(x, y)\right)\right|},
$$

for any $x, y \in \mathbb{R}^{n}($ with $x \neq y)$ and any choice of $Z_{1}, \ldots, Z_{r} \in\left\{X_{1}^{x}, \ldots, X_{m}^{x}, X_{1}^{y}, \ldots, X_{m}^{y}\right\}$. In particular, for every fixed $x \in \mathbb{R}^{n}$ we have

$$
\lim _{|y| \rightarrow \infty} Z_{1} \cdots Z_{r} \Gamma(x ; y)=0
$$

(III). Suppose that $n>2$. Then one has

$$
C^{-1} \frac{d_{X}(x, y)^{2}}{\left|B_{X}\left(x, d_{X}(x, y)\right)\right|} \leq \Gamma(x ; y) \leq C \frac{d_{X}(x, y)^{2}}{\left|B_{X}\left(x, d_{X}(x, y)\right)\right|},
$$

for any $x, y \in \mathbb{R}^{n}($ with $x \neq y)$. Here $C \geq 1$ is a structural constant.

\footnotetext{
${ }^{2}$ Note that $\mathcal{L}$ is formally selfadjoint on test functions, due to simple arguments based on (H.1).
} 
(IV). Suppose that $n=2$. For every compact set $K \subseteq \mathbb{R}^{n}$ there exist structural constants $c_{1}, c_{2}>0$ and real numbers $R_{1}, R_{2}>0$ (all depending on $K$ ) such that

$$
c_{1} \log \left(\frac{R_{1}}{d_{X}(x, y)}\right) \leq \Gamma(x ; y) \leq c_{2} \frac{d_{X}(x, y)^{2}}{\left|B_{X}\left(x, d_{X}(x, y)\right)\right|} \cdot \log \left(\frac{R_{2}}{d_{X}(x, y)}\right),
$$

uniformly for $x \neq y$ in $K$. Moreover, for every fixed pole $x \in \mathbb{R}^{n}$, there exist constants $\gamma_{1}(x), \gamma_{2}(x)>$ 0 and $0<\varepsilon(x)<1$ such that

$$
\gamma_{1}(x) F(x, y) \leq \Gamma(x ; y) \leq \gamma_{2}(x) F(x, y),
$$

for any $y$ such that $0<d_{X}(x, y)<\varepsilon(x)$, where

$$
F(x, y)= \begin{cases}\log \left(\frac{1}{d_{X}(x, y)}\right) & \text { if } f_{2}(x)>0, \\ \frac{d_{X}(x, y)^{2}}{\left|B_{X}\left(x, d_{X}(x, y)\right)\right|} & \text { if } f_{2}(x)=0 .\end{cases}
$$

Here $f_{2}$ is the nonnegative function which will be defined in Theorem B. In the case $f_{2}(x)=0$, the estimate of $\Gamma(x ; y)$ holds true with $\varepsilon(x)=1 / 2$ and $\gamma_{1}(x)$ independent of $x$; in this case, $F(x, y)$ diverges like $d_{X}(x, y)^{2-k}$, for some $k \in\{3, \ldots, q\}$ which depends on $x$.

(V). In particular, for any $n \geq 2, \Gamma(x ; \cdot)$ has a pole at $x \in \mathbb{R}^{n}$, i.e.,

$$
\lim _{y \rightarrow x} \Gamma(x ; y)=\infty \text {. }
$$

Remark 1.4. As a matter of facts, most of the results in Theorem 1.3 can be naturally extended to homogeneous Hörmander operators of the kind

$$
\mathcal{L}=\sum_{i=1}^{m} X_{i}^{2}+X_{0},
$$

where $X_{1}, \ldots, X_{m}$ are $\delta_{\lambda}$-homogeneous of degree 1 and $X_{0}$ (the drift) is $\delta_{\lambda}$-homogeneous of degree 2 (see Theorem 9.4 for the precise statement). In order to keep more readable our presentation, we have only briefly sketched in Section 9 the adjustments of the theory necessary to cover this more general setting, while the core of the paper is written for sum of squares.

Let us now say a few words about the techniques used in the proofs of our results, thus seizing the opportunity to put our paper in the context of the existing literature. Our first step is to combine the integral representation of $\Gamma$ given in (1.5), and similar representation formulas which will be established for the derivatives of $\Gamma$ (those in (I) of Theorem 1.3), with the global growth estimates satisfied, for homogeneity reasons, by $\Gamma_{\mathbb{G}}$ and its derivatives: this combination gives

$$
\left|Z_{1} \cdots Z_{r} \Gamma(x ; y)\right| \leq c_{r} \int_{\mathbb{R}^{p}} d_{\widetilde{X}}^{2-Q-r}\left((x, 0)^{-1} *(y, \eta)\right) \mathrm{d} \eta,
$$

for every $x, y \in \mathbb{R}^{n}, x \neq y$, where $d_{\widetilde{X}}$ is the CC-distance induced in the Carnot group $\mathbb{R}^{N}$ by the lifted vector fields $\widetilde{X}_{1}, \ldots, \widetilde{X}_{m}$. Once this is accomplished, we shall bound the above integral by means of two deep results related to the geometry of Hörmander vector fields and established in the papers [27] and 29, here suitably extended to a global version (thanks to the underlying $\delta_{\lambda}$-homogenous structure): see Theorems B and C in Section 2 .

One could object to our procedure the fact that local estimates for $\Gamma$ and its derivatives are also contained in [27, 29. So one could think to derive global estimates from the existing local estimates, just by dilation arguments. However, what remains a bit unclear in those papers is which object referred to as $\Gamma$ is actually being estimated. For instance, in [27, Thm. 5] a conditional statement is proved, saying that if, in the space of the lifted variables, a kernel $\widetilde{\Gamma}$ satisfies an estimate of the kind

$$
\widetilde{\Gamma}((x, \xi) ;(y, \eta)) \leq c d_{\widetilde{X}}^{2-Q}((x, \xi),(y, \eta)),
$$

then the kernel $\Gamma$ that we get by locally saturating the lifted variables similarly to (1.5) satisfies local estimates in $\mathbb{R}^{n}$ of the kind

$$
\Gamma(x ; y) \leq c \frac{d_{X}^{2}(x, y)}{\left|B_{X}\left(x, d_{X}(x, y)\right)\right|}
$$


(with analogous statements about the derivatives of $\widetilde{\Gamma}$ and $\Gamma$ ). In 27 the alluded kernel $\widetilde{\Gamma}$ is the parametrix for the lifted operator constructed in the paper 28 (no fundamental solution is built in [28). However, the kernel $\Gamma$ obtained by this procedure is hopefully a local parametrix for $\mathcal{L}$, but not necessarily a fundamental solution. Also, it is a function defined only locally, and in a non-unique way. Actually, to produce a true local fundamental solution saturating a parametrix in a lifted space, a hard extra-work is needed (see e.g., 16]). In contrast with this, the function $\Gamma$ that we consider is a uniquely defined, global, fundamental solution for $\mathcal{L}$; for this object, and its derivatives, global estimates are proved, together with representation formulas which also contain some additional information, not limited to the size of these functions. An example of the relevance of this last statement will be given in Section 8 see Theorem 8.1-(iii) and Remark 8.3

We also note that the estimates that we shall prove for the derivatives of $\Gamma(x ; y)$ apply to derivatives of any order, with respect to both variables $x$ and $y$. As we shall see, the case of mixed derivatives requires a more delicate proof (see Lemma 4.3). On the other hand, this bound has interesting consequences, as we shall see in Section 8 (Theorem 8.2). Incidentally, in [27, p. 141] a proof is written only for the basic estimate of $\widetilde{\Gamma}$, while the proof of the derivative estimates is left to the reader.

Once the results in Theorem 1.3 are established, we consider some possible applications. Firstly, in Section 7 we deal with potential-theoretic properties of $\mathcal{L}$. Indeed, the estimates of $\Gamma$ and the presence of a blowing-up pole (see (V) in Theorem 1.3) allow us to verify, for our operators $\mathcal{L}$, all the axioms of potential theory required for the analysis contained in the series of papers [1, 2, 4, 12. Secondly, in Section 8 we shall show that the kernel

$$
k(x, y)=X_{i}^{x} X_{j}^{x} \Gamma(x ; y)
$$

satisfies, globally in $\mathbb{R}^{n}$, the so-called standard estimates of singular integrals, together with a suitable cancelation property, with respect to both variables. These facts will be proved as a consequence of the estimates on second and third order (pure or mixed) derivatives of $\Gamma$, together with the explicit integral representation formula of $k(x, y)$ in terms of the homogeneous fundamental solution $\widetilde{\Gamma}$ on the Carnot group $\mathbb{R}^{N}$. These properties of $k(x, y)$ could be a starting point to prove global Sobolev estimates for solutions to $L u=f$, both for our operator $\mathcal{L}$ and for more general classes of non-variational operators $\sum_{i, j} a_{i j}(x) X_{i} X_{j}$ modeled on our vector fields (with low regular $a_{i, j}$ 's and $A=\left(a_{i, j}(x)\right)$ in some class of ellipticity). This theory will be developed elsewhere.

\section{Notations AND A REVIEW OF KNOWN RESUlts}

In what follows, we denote by $d_{X}$ the Carnot-Carathéodory (CC, shortly) distance associated with the set of Hörmander vector fields $X=\left\{X_{1}, \ldots, X_{m}\right\}$, that is,

$$
d_{X}(x, y):=\inf \{r>0: \text { there exists } \gamma \in C(r) \text { with } \gamma(0)=x \text { and } \gamma(1)=y\},
$$

where $C(r)$ is the set of the absolutely continuous maps $\gamma:[0,1] \rightarrow \mathbb{R}^{n}$ satisfying (a.e. on $[0,1]$ )

$$
\gamma^{\prime}(t)=\sum_{j=1}^{m} a_{j}(t) X_{j}(\gamma(t)), \quad \text { with }\left|a_{j}(t)\right| \leq r \text { for all } j=1, \ldots, m .
$$

Given any $x \in \mathbb{R}^{n}$ and $r>0$, we denote by $B_{X}(x, r)$ the $d_{X}$-ball of center $x$ and radius $r$. Without risk of confusion, $|\cdot|$ will denote the Lebesgue measure in $\mathbb{R}^{n}$ (whatever the $n$ ).

Thanks to (H.1), $d_{X}$ and $B_{X}$ enjoy the homogeneity properties:

$$
\begin{aligned}
& d_{X}\left(\delta_{\lambda}(x), \delta_{\lambda}(y)\right)=\lambda d_{X}(x, y), \\
& y \in B_{X}(x, r) \Longleftrightarrow \delta_{\lambda}(y) \in B_{X}\left(\delta_{\lambda}(x), \lambda r\right), \\
& \left|B_{X}\left(\delta_{\lambda}(x), \lambda r\right)\right|=\lambda^{q}\left|B_{X}(x, r)\right|,
\end{aligned}
$$

for any $x, y \in \mathbb{R}^{n}$ and any $\lambda, r>0$. Moreover, it is well known from 27] that, for $x$ in some compact set and for $r$ small enough, one has the doubling property

$$
\left|B_{X}(x, 2 r)\right| \leq C_{d}\left|B_{X}(x, r)\right| .
$$


As a matter of fact, by a homogeneity argument based on (2.2), the above doubling property holds true for every $x \in \mathbb{R}^{n}$ and every $r>0$ (see, e.g., 4], or see Section 3).

In the next sections, $d_{\widetilde{X}}$ will stand for the CC-distance associated with the system of vector fields $\widetilde{X}=\left\{\widetilde{X}_{1}, \ldots, \widetilde{X}_{m}\right\}$ introduced in point (1) of Theorem A. Since the $\widetilde{X}_{j}$ 's lift the $X_{j}$ 's (and all these vector fields are homogeneous with respect to appropriate dilations) it is known that (here, $\pi_{n}$ is the projection of $\mathbb{R}^{N}=\mathbb{R}^{n} \times \mathbb{R}^{p}$ onto $\mathbb{R}^{n}$ )

$$
\begin{aligned}
& d_{X}(x, y) \leq d_{\widetilde{X}}((x, \xi),(y, \eta)), \quad \text { for any } x, y \in \mathbb{R}^{n} \text { and } \xi, \eta \in \mathbb{R}^{p}, \\
& \pi_{n}\left(B_{\widetilde{X}}((x, \xi), r)\right)=B_{X}(x, r), \quad \text { for any } x \in \mathbb{R}^{n}, \xi \in \mathbb{R}^{p} \text { and } r>0 .
\end{aligned}
$$

Furthermore, since the $\widetilde{X}_{j}$ 's are left-invariant on the group $\mathbb{G}=\left(\mathbb{R}^{N}, *\right)$, one has

$$
d_{\widetilde{X}}\left(z, z^{\prime}\right)=d_{\widetilde{X}}\left(0, z^{-1} * z^{\prime}\right), \quad \text { for every } z, z^{\prime} \in \mathbb{G} .
$$

By an abuse of notation, we shall systematically denote $d_{\widetilde{X}}(0, \cdot)$ by $d_{\widetilde{X}}$. Simple arguments on homogeneous groups also show that

$$
\left|B_{\widetilde{X}}(z, r)\right|=\omega_{Q} r^{Q}, \quad \text { where } \omega_{Q}=\left|B_{\widetilde{X}}(0,1)\right| .
$$

It can also be proved that $\mathbb{G}$ is a homogeneous group with dilations

$$
D_{\lambda}: \mathbb{R}^{N}=\mathbb{R}^{n} \times \mathbb{R}^{p} \longrightarrow \mathbb{R}^{N}, \quad D_{\lambda}(x, \xi)=\left(\delta_{\lambda}(x), E_{\lambda}(\xi)\right),
$$

where $E_{\lambda}(\xi)=\left(\lambda^{\tau_{1}} \xi, \ldots, \lambda^{\tau_{p}} \xi_{p}\right)$ for suitable integers $1 \leq \tau_{1} \leq \cdots \leq \tau_{p}$. We point out that the exponents of $D_{\lambda}$ are not increasingly ordered (but this is true of the $\sigma_{i}$ 's and of $\tau_{j}$ 's separately). This will cause some subtleties in handling the group structure of $\mathbb{G}$ (see, e.g., Lemma 4.5).

In this paper we shall make use of the following two theorems, concerning the volume of the $d_{X}$-metric balls (Theorem B) and concerning the relation between the volumes of lifted and unlifted balls (Theorem C). We shall derive them in Section 3 via a local-to-global homogeneity-argument, starting from their local counterparts. These local counterparts will be obtained from the deep investigations on subelliptic distances carried out by Nagel, Stein, Wainger [27. Theorem 1], and by Sánchez-Calle [29, Theorem 4] (see also [27, Lemma 3.2] and Jerison [25]).

Theorem B. Let $q$ be as in (1.2). For any $k \in\{n, \ldots, q\}$ there exists a function $f_{k}: \mathbb{R}^{n} \rightarrow \mathbb{R}$ which is continuous, nonnegative and $\delta_{\lambda}$-homogeneous of degree $q-k$, and there exist structural constants $\gamma_{1}, \gamma_{2}>0$ such that

$$
\gamma_{1} \sum_{k=n}^{q} f_{k}(x) \rho^{k} \leq\left|B_{X}(x, \rho)\right| \leq \gamma_{2} \sum_{k=n}^{q} f_{k}(x) \rho^{k},
$$

for every $x \in \mathbb{R}^{n}$ and every $\rho>0$. Moreover, $f_{q}(x)$ is constant in $x$, and strictly positive.

Remark 2.1 (Explicit form of the $f_{k}$ 's). In the following, we shall occasionally need the explicit form of the functions $f_{k}$. To explain their definition (according to [27]), we need to introduce some more notation, that will also be useful for other reasons. For a multi-index

$$
I=\left(i_{1}, \ldots, i_{k}\right), \quad \text { with } i_{1}, \ldots, i_{k} \in\{1,2, \ldots, m\},
$$

let us define (when $k=1) X_{[I]}=X_{i_{1}}$, and (when $k>1$ )

$$
X_{[I]}:=\left[\left[\left[X_{i_{1}}, X_{i_{2}}\right], X_{i_{3}}\right], \ldots, X_{i_{k}}\right] \text {; }
$$

we also define the weight (or length) of $I$ as $|I|=k$. At any fixed point $x \in \mathbb{R}^{n}$, let us consider a basis of $\mathbb{R}^{n}$ consisting in a set $\left\{X_{[I]}(x): I \in \mathcal{A}\right\}$ (where $\mathcal{A}$ is a set of $n$ multi-indices) of $n$ commutators evaluated at $x$, and let us arrange these $n$ vectors in an $n \times n$ matrix which we denote by:

$$
\mathcal{B}(x)=\left(X_{[I]}(x)\right)_{I \in \mathcal{A}} .
$$

The weight of this basis will be, by definition,

$$
|\mathcal{B}(x)|=\sum_{I \in \mathcal{A}}|I|
$$


Then, according to [27, the functions $f_{k}$ appearing in Theorem B are equal to

$$
f_{k}(x)=\sum_{|\mathcal{B}(x)|=k}|\operatorname{det}(\mathcal{B}(x))|,
$$

where the sum is taken over all the possible bases of $\mathbb{R}^{n}$ having weight $k$.

Theorem C. There exist constants $\kappa \in(0,1)$ and $c_{1}, c_{2}>0$ such that, for every $x \in \mathbb{R}^{n}, \xi \in \mathbb{R}^{p}$ and $r>0$ one has the following estimates:

$$
\begin{aligned}
& \left|\left\{\eta \in \mathbb{R}^{p}:(y, \eta) \in B_{\widetilde{X}}((x, \xi), r)\right\}\right| \leq c_{1} \frac{\left|B_{\widetilde{X}}((x, \xi), r)\right|}{\left|B_{X}(x, r)\right|}, \quad \text { for all } y \in \mathbb{R}^{n}, \\
& \left|\left\{\eta \in \mathbb{R}^{p}:(y, \eta) \in B_{\widetilde{X}}((x, \xi), r)\right\}\right| \geq c_{2} \frac{\left|B_{\widetilde{X}}((x, \xi), r)\right|}{\left|B_{X}(x, r)\right|}, \quad \text { for all } y \in B_{X}(x, \kappa r) .
\end{aligned}
$$

Remark 2.2. As a matter of fact, the local version of Theorem $\mathrm{C}$ is proved in [27, 29] (see also 25]) in a slightly different framework: namely [27, 29] assume that the vector fields in $\widetilde{X}$ lift those in $X$ in the sense of the lifting by Rothschild-Stein [28. Nevertheless, scrutinizing the proof of Theorem $\mathrm{C}$ in its local version (as written in detail, e.g., in [15, Chap. 10]), one can see that the estimates in (2.8)-(2.9) hold true for our vector fields as well, where $\widetilde{X}$ lift $X$ in the sense of Folland [20.

Namely, most of the proof of Theorem $\mathrm{C}$ relies on the deep properties established by Nagel, Stein, Wainger 27] for every system of Hörmander vector fields, while the only special properties of the vector fields $\widetilde{X}_{i}$ which are exploited are the following:

(1) the vector field $\widetilde{X}_{i}$ projects onto $X_{i}$ for any $i$;

(2) if a family of commutators of $\left\{\widetilde{X}_{[I]}\right\}_{I \in \mathcal{A}}$ is a basis of $\mathbb{R}^{N}$ at some point of $\mathbb{R}^{N}$, then the same is true at every other point;

(3) if any two families of commutators $\left\{\widetilde{X}_{[I]}\right\}_{I \in \mathcal{A}}$ and $\left\{\widetilde{X}_{\left[I^{\prime}\right]}\right\}_{I^{\prime} \in \mathcal{A}^{\prime}}$ are bases of $\mathbb{R}^{N}$ at some point of $\mathbb{R}^{N}$, then it holds that

$$
\sum_{I \in \mathcal{A}}|I|=\sum_{I^{\prime} \in \mathcal{A}^{\prime}}\left|I^{\prime}\right|
$$

In our setting, property (1) holds true by our very definition of lifting (1.4); properties (2) and (3) (fulfilled by the Rothschild-Stein's lifted vector fields as they are free vector fields) hold true in our case since the $\widetilde{X}_{i}$ 's are the Lie-generators of a stratified Carnot group $\mathbb{G}$ (see Theorem A).

More precisely, property (2) is a consequence of the left invariance (see [11, Prop. C.5]), while property (3) is a consequence of the stratification of the Lie algebra. Namely, assume that

$$
x=\left\{\widetilde{X}_{[I]}\right\}_{I \in \mathcal{A}}
$$

is a basis of $\mathbb{R}^{N}$ at some point in space: then $X$ is also a basis of Lie( $\mathbb{G}$ ) (see again [11, Prop. C.5]); moreover, by grouping together the elements of $X$ with the same $D_{\lambda}$-homogeneity, one gets bases of the layers of the stratification of $\operatorname{Lie}(\mathbb{G})$. It then suffices to apply [13, Prop. 2.2.8], ensuring that $\sum_{I \in \mathcal{A}}|I|$ is nothing but the so-called $D_{\lambda}$-homogeneous dimension of $\mathbb{G}$, which is independent of $\mathcal{X}$.

A last remark is in order. Our statement of Theorem $\mathrm{C}$ involves lifted balls $B_{\widetilde{X}}((x, \xi), r)$, while the analogous (local) statement in [27] deals with lifted balls centred at points of the form $(x, 0)$. However, this latter choice is immaterial, motivated (as appears by a close inspection of the proof in [27]) by notational convenience.

\section{LOCAL TO GLOBAL VIA HOMOGENEITY}

The following fact is a very simple consequence of homogeneity; we shall use it so many times that we provide it in details for the sake of reference convenience.

Remark 3.1. Let $m \in \mathbb{N}$; let $\left\{M_{\lambda}\right\}_{\lambda>0}$ be the family of dilations of $\mathbb{R}^{m}$ defined by

$$
M_{\lambda}\left(w_{1}, \ldots, w_{m}\right)=\left(\lambda^{\mu_{1}} w_{1}, \ldots, \lambda^{\mu_{m}} w_{m}\right),
$$


where $\mu_{1}, \ldots, \mu_{m}$ are fixed positive real numbers. Let $\Omega \subseteq \mathbb{R}^{m}$ be closed under $\left\{M_{\lambda}\right\}_{\lambda}$, i.e.,

$$
M_{\lambda}(w) \in \Omega \text { for every } w \in \Omega \text { and every } \lambda>0 .
$$

Suppose that $F, G: \Omega \rightarrow \mathbb{R}$ are two $M_{\lambda}$-homogeneous functions of the same degree, say $\alpha$, i.e.,

$$
\left\{\begin{array}{l}
F\left(M_{\lambda}(w)\right)=\lambda^{\alpha} F(w) \\
G\left(M_{\lambda}(w)\right)=\lambda^{\alpha} G(w),
\end{array} \quad \text { for every } w \in \Omega \text { and every } \lambda>0 .\right.
$$

Finally, suppose that there exists a neighborhood $O$ of $0 \in \mathbb{R}^{m}$ such that $O \cap \Omega \neq \varnothing$ and $F \leq G$ on $O \cap \Omega$; then $F \leq G$ on $\Omega$. Indeed, let $w \in \Omega$ be arbitrary; then there exists a small $\lambda>0$ such that $M_{\lambda}(w) \in O \cap \Omega$ (this follows from (3.1) and since $M_{\lambda}(w) \rightarrow 0 \in \mathbb{R}^{m}$ as $\lambda \rightarrow 0^{+}$). As $F \leq G$ on $O \cap \Omega$ we infer that $F\left(M_{\lambda}(w)\right) \leq G\left(M_{\lambda}(w)\right)$; due to the $M_{\lambda}$-homogeneity of $F$ and $G$, this is equivalent to $\lambda^{\alpha} F(w) \leq \lambda^{\alpha} G(w)$. Canceling out $\lambda^{\alpha}>0$, this gives $F(w) \leq G(w)$.

A completely analogous result holds true if we replace " $F \leq G$ " with " $F=G$ " or " $F<G$."

As a first application of Remark 3.1, we prove the global doubling inequality (2.3): indeed, by classical results in [27, one knows that there exist a constant $C_{d}>0$, a neighborhood $U_{0}$ of $0 \in \mathbb{R}^{n}$ and some $r_{0}>0$ such that $\left|B_{X}(x, 2 r)\right| \leq C_{d}\left|B_{X}(x, r)\right|$ for every $x \in U_{0}$ and every $r \in\left(0, r_{0}\right)$. Then we apply Remark 3.1 with the choices $m=n+1, \Omega=\mathbb{R}^{n} \times(0, \infty)$,

$$
M_{\lambda}(x, r)=\left(\delta_{\lambda}(x), \lambda r\right) \text { for } x \in \mathbb{R}^{n}, r>0 \text { and } \lambda>0,
$$

and with the functions $F(x, r)=\left|B_{X}(x, 2 r)\right|$ and $G(x, r)=C_{d}\left|B_{X}(x, r)\right|$. These choices satisfy the assumptions in Remark 3.1 since $\Omega$ is invariant under $\left\{M_{\lambda}\right\}_{\lambda}, F \leq G$ on $U_{0} \times\left(0, r_{0}\right)$ (which is of the form $O \cap \Omega$ for some neighborhood $O$ of $0 \in \mathbb{R}^{m}$ ) and since $F$ and $G$ are both $M_{\lambda}$-homogeneous of degree $q$, due to (2.2).

Another application of Remark 3.1 is the following:

Remark 3.2. We prove that, due to assumptions (H.1) and (H.2), the validity of Hörmander's rank condition at 0 implies its validity at any other point $x \in \mathbb{R}^{n}$. Indeed, it is easy to check that, by (H.1), the vector field $X_{[I]}$ is $\delta_{\lambda}$-homogeneous of degree $|I|$, which is equivalent to

$$
X_{[I]}\left(\delta_{\lambda}(x)\right)=\lambda^{-|I|} \delta_{\lambda}\left(X_{[I]}(x)\right), \quad \forall \lambda>0, x \in \mathbb{R}^{n} .
$$

Next, we observe that the iterated (left nested) brackets $X_{[I]}$ span Lie(X). Hence, by (H.2), we can find a family $X_{\left[I_{1}\right]}, \ldots, X_{\left[I_{n}\right]}$ such that $X_{\left[I_{1}\right]}(0), \ldots, X_{\left[I_{n}\right]}(0)$ is a basis of $\mathbb{R}^{n}$. Thus, the function

$$
x \mapsto F(x):=\operatorname{det}\left(X_{\left[I_{1}\right]}(x) \cdots X_{\left[I_{n}\right]}(x)\right)
$$

is non-null on a neighborhood $O$ of $0 \in \mathbb{R}^{n}$. If we show that $F$ is $\delta_{\lambda}$-homogeneous, then Remark 3.1 will prove that $F(x) \neq 0$ for every $x \in \Omega:=\mathbb{R}^{n}$. The $\delta_{\lambda}$-homogeneity of $F$ can be proved as follows:

$$
\begin{aligned}
F\left(\delta_{\lambda}(x)\right) & \stackrel{\sqrt{3.2}}{=} \operatorname{det}\left(\lambda^{-\left|I_{1}\right|} \delta_{\lambda}\left(X_{\left[I_{1}\right]}(x)\right) \cdots \lambda^{-\left|I_{n}\right|} \delta_{\lambda}\left(X_{\left[I_{n}\right]}(x)\right)\right) \\
& =\lambda^{-\left|I_{1}\right|-\cdots-\left|I_{n}\right|} \operatorname{det}\left(\delta_{\lambda}\left(X_{\left[I_{1}\right]}(x)\right) \cdots \delta_{\lambda}\left(X_{\left[I_{n}\right]}(x)\right)\right) \\
& =\lambda^{q-\left|I_{1}\right|-\cdots-\left|I_{n}\right|} \operatorname{det}\left(X_{\left[I_{1}\right]}(x) \cdots X_{\left[I_{n}\right]}(x)\right)=\lambda^{q-\left|I_{1}\right|-\cdots-\left|I_{n}\right|} F(x) .
\end{aligned}
$$

Remark 3.3. As a matter of fact, the CC-distance $d_{X}$ in (2.1) is not the unique distance one can attach to the Hörmander family $X$ : for instance, following the notation in Remark 2.2 , one can deal with the so-called subelliptic distance

$$
\rho(x, y):=\inf \left\{r>0: \text { there exists } \gamma \in C^{\prime}(r) \text { with } \gamma(0)=x \text { and } \gamma(1)=y\right\}
$$

where $C^{\prime}(r)$ is the set of absolutely continuous maps $\gamma:[0,1] \rightarrow \mathbb{R}^{n}$ satisfying (a.e. on $[0,1]$ )

$$
\gamma^{\prime}(t)=\sum_{|I| \leq \sigma_{n}} a_{I}(t) X_{[I]}(\gamma(t)), \quad \text { with }\left|a_{I}(t)\right| \leq r^{|I|} \text { for all } I .
$$

We remind that $\sigma_{n}$ is the largest length of some non-vanishing commutator $X_{[I]}$ (see Remark 1.2). On account of the results in [27, Section 1.4], $\rho$ and $d_{X}$ are locally equivalent:

$$
c_{1} d_{X}(x, y) \leq \rho(x, y) \leq c_{2} d_{X}(x, y),
$$


for every $x, y$ in some neighborhood $U_{0}$ of the origin, and some constants $c_{1}, c_{2}>0$. Moreover, it is not difficult to check that $\gamma \in C^{\prime}(r)$ if and only if $\delta_{\lambda} \circ \gamma \in C^{\prime}(\lambda r)$; as a consequence,

$$
\rho\left(\delta_{\lambda}(x), \delta_{\lambda}(y)\right)=\lambda \rho(x, y) \text { for every } x, y \in \mathbb{R}^{n} \text { and every } \lambda>0 .
$$

Starting from (3.3)-(3.4), and using Remark 3.1 one can prove that (3.3) holds for every $x, y \in \mathbb{R}^{n}$. The global version of (3.3) implies the following inclusions:

$$
B_{\rho}\left(x, c_{1} r\right) \subseteq B_{X}(x, r) \subseteq B_{\rho}\left(x, c_{2} r\right), \quad \text { for all } x \in \mathbb{R}^{n} \text { and } r>0 .
$$

We shall now use Remarks 3.1]3.3 in deriving Theorems B and C from their local counterparts.

Proof of Theorem B. We start from the notable estimate proved in [27, Theorem 1]: there exist a neighborhood $U_{0}$ of the origin in $\mathbb{R}^{n}$, a real $r_{0}^{\prime}>0$ and two constants $\gamma_{1}^{\prime}, \gamma_{2}^{\prime}>0$ such that

$$
\gamma_{1}^{\prime} \Lambda(x, r) \leq\left|B_{\rho}(x, r)\right| \leq \gamma_{2}^{\prime} \Lambda(x, r), \quad \text { for every } x \in U_{0} \text { and every } 0<r \leq r_{0}^{\prime},
$$

where (following the notation in Remark 2.2 for $I_{j}, X_{\left[I_{j}\right]}$ and $\left|I_{j}\right|$ )

$$
\Lambda(x, r):=\sum_{B=\left(I_{1}, \ldots, I_{n}\right)}\left|\operatorname{det}\left(X_{\left[I_{1}\right]}(x) \cdots X_{\left[I_{n}\right]}(x)\right)\right| r^{\left|I_{1}\right|+\cdots+\left|I_{n}\right|},
$$

and the sum runs over all the $n$-tuples $B$ of multi-indexes $I_{j}$ 's with $\left|I_{j}\right| \leq \sigma_{n}$. We now observe that, by definition, $\left|I_{1}\right|+\cdots+\left|I_{n}\right| \geq n$; on the other hand, from Remark 3.3 we know that

$$
x \mapsto \operatorname{det}\left(X_{\left[I_{1}\right]}(x) \cdots X_{\left[I_{n}\right]}(x)\right)
$$

is $\delta_{\lambda}$-homogeneous of degree $q-\left(\left|I_{1}\right|+\cdots+\left|I_{n}\right|\right)$. Hence, the latter function (which is smooth on $\left.\mathbb{R}^{n}\right)$ must vanish whenever $\left|I_{1}\right|+\cdots+\left|I_{n}\right|>q$ and it is constant when the equality holds.

Gathering together all these facts, we can reorder the sum in the right-hand side of (3.7) with respect to $\left|I_{1}\right|+\cdots+\left|I_{n}\right|$, obtaining the representation

$$
\Lambda(x, r)=\sum_{k=n}^{q} f_{k}(x) r^{k}
$$

where $f_{k}$ is a nonnegative continuous function on $\mathbb{R}^{n}$ which is $\delta_{\lambda}$-homogeneous of degree $q-k$. In particular, $f_{q}$ is constant and

$$
\Lambda\left(\delta_{\lambda}(x), \lambda r\right)=\lambda^{q} \Lambda(x, r), \quad \text { for every } x \in \mathbb{R}^{n} \text {, every } r, \lambda>0 .
$$

On account of (3.5) and (3.6), we get the estimate

$$
\gamma_{1} \sum_{k=n}^{q} f_{k}(x) r^{k} \leq\left|B_{X}(x, r)\right| \leq \gamma_{2} \sum_{k=n}^{q} f_{k}(x) r^{k}, \quad \text { for } x \in U_{0} \text { and } 0<r \leq r_{0},
$$

for some structural positive constants $\gamma_{1}, \gamma_{2}$ and $r_{0}$. One can pass from the local (3.9) to the global (2.7) by using Remark 3.1 and the fact that all members of (3.9) are homogeneous of degree $q$ with respect to the dilations $(x, r) \mapsto\left(\delta_{\lambda}(x), \lambda r\right)$. Finally, taking $x=0$ and $r=1$ in (2.7), we infer that the constant $f_{q}$ is strictly positive, since $f_{k}(0)=0$ for all $n \leq k \leq q-1$ (which is a consequence of the $\delta_{\lambda}$-homogeneity of $f_{k}$ of degree $q-k$ ).

Finally we provide the

Proof of Theorem $C$. The deep result proved in [29, Theorem 4] provides a local version of (2.8)(2.9). As for (2.8), one can pass from local to global via an application of Remark 3.1, once noticed that the members in (2.8) are homogeneous of degree $Q-q$ with respect to (see also (4.16) )

$$
(x, \xi, y, r) \mapsto\left(\delta_{\lambda}(x), E_{\lambda}(\xi), \delta_{\lambda}(y), \lambda r\right) .
$$

In this argument it may help observing that

$$
\left\{\eta \in \mathbb{R}^{p}:\left(\delta_{\lambda}(y), \eta\right) \in B_{\widetilde{X}}\left(\left(\delta_{\lambda}(x), E_{\lambda}(\xi)\right), \lambda r\right)\right\}=E_{\lambda}\left(\left\{\eta^{\prime} \in \mathbb{R}^{p}:\left(y, \eta^{\prime}\right) \in B_{\widetilde{X}}((x, \xi), r)\right\}\right) .
$$

As for (2.9), one chooses $\lambda>0$ so small that

$$
\left|\left\{\eta \in \mathbb{R}^{p}:\left(\delta_{\lambda}(y), \eta\right) \in B_{\widetilde{X}}\left(\left(\delta_{\lambda}(x), E_{\lambda}(\xi)\right), \lambda r\right)\right\}\right| \geq c_{2} \frac{\left|B_{\widetilde{X}}\left(\left(\delta_{\lambda}(x), E_{\lambda}(\xi)\right), \lambda r\right)\right|}{\left|B_{X}\left(\delta_{\lambda}(x), \lambda r\right)\right|},
$$


holds true when $\delta_{\lambda}(y) \in B_{X}\left(\delta_{\lambda}(x), \kappa \lambda r\right)$ (here, $\kappa>0$ is the same as in [29, Theorem 4]). From this, by applying (2.2) and by arguing as above, one gets the desired (2.9).

\section{Global upper estimates of $\Gamma$ AND its DeRivatives}

In this section we tacitly inherit the notations in Section 2, and the assumptions (H.1)-to(H.3); in particular $q$ is always assumed to be larger than 2. This assumption is not restrictive, as the case $q=2$ boils down to the well-known case when $\mathcal{L}$ is a (strictly) elliptic operator in $\mathbb{R}^{2}$ with constant coefficients. Indeed $q=2$ implies that $n=2$ and $\delta_{\lambda}\left(x_{1}, x_{2}\right)=\left(\lambda x_{1}, \lambda x_{2}\right)$; as a consequence, since $X_{1}, \ldots, X_{m}$ are linearly independent Hörmander vector fields, $\delta_{\lambda}$-homogeneous of degree 1 , one necessarily has $m=2$ and

$$
X_{1}=a \partial_{x_{1}}+b \partial_{x_{2}}, \quad X_{2}=\alpha \partial_{x_{1}}+\beta \partial_{x_{2}} \quad \text { with } a \beta-b \alpha \neq 0
$$

The aim of the present section is to prove the following main result:

Theorem 4.1 (Global estimates of $\Gamma$ and its derivatives). Let $\Gamma$ be the fundamental solution of $\mathcal{L}$ in (1.5); we also assume that $n>2$. Then, for any integer $r \geq 0$, there exists $C>0$ (depending on $r$ and on the set $\left.X=\left\{X_{1}, \ldots, X_{m}\right\}\right)$ such that

$$
\left|Z_{1} \cdots Z_{r} \Gamma(x ; y)\right| \leq C \frac{d_{X}(x, y)^{2-r}}{\left|B_{X}\left(x, d_{X}(x, y)\right)\right|},
$$

holding true for any $x, y \in \mathbb{R}^{n}($ with $x \neq y)$ and any choice of

$$
Z_{1}, \ldots, Z_{r} \in\left\{X_{1}^{x}, \ldots, X_{m}^{x}, X_{1}^{y}, \ldots, X_{m}^{y}\right\}
$$

where superscripts denote the variable with respect to which differentiation is performed. In particular, for every fixed $x \in \mathbb{R}^{n}$ we have

$$
\lim _{|y| \rightarrow \infty} Z_{1} \cdots Z_{r} \Gamma(x ; y)=0 .
$$

The estimate (4.1) results from an integral representation of $Z_{1} \cdots Z_{r} \Gamma(x ; y)$ (see precisely Lemma 4.3), which seems to have an interest in its own.

The case $n=2$, which is not comprised in Theorem 4.1, will be investigated in Section 6 .

Remark 4.2. The function $H(x, y)$ in the right-hand side of (4.1) is not symmetric (in $x, y$ ) as it stands; however, one can recognize that it is equivalent (up to a structural constant) to the function $H(y, x)$. Indeed, one has the following computation based on the doubling inequality (2.3) (and on the trivial inclusion $\left.B_{X}\left(y, d_{X}(x, y)\right) \subseteq B_{X}\left(x, 2 d_{X}(x, y)\right)\right)$ :

$$
H(x, y)=\frac{d_{X}(x, y)^{2-r}}{\left|B_{X}\left(x, d_{X}(x, y)\right)\right|}=\frac{d_{X}(y, x)^{2-r}}{\left|B_{X}\left(x, d_{X}(y, x)\right)\right|} \leq C_{d} \frac{d_{X}(y, x)^{2-r}}{\left|B_{X}\left(y, d_{X}(y, x)\right)\right|}=C_{d} H(y, x) .
$$

The proof of Theorem 4.1 is long and requires several preliminary results, the first of which is the following lemma, where the role of mixed derivatives is unexpectedly delicate.

Lemma 4.3. For any $s, t \geq 1$, and any choice of $i_{1}, \ldots, i_{s}, j_{1}, \ldots, j_{t} \in\{1, \ldots, m\}$, we have the following representation formulas (holding true for $x \neq y$ in $\mathbb{R}^{n}$ ):

$$
\begin{aligned}
& X_{i_{1}}^{y} \cdots X_{i_{s}}^{y}(\Gamma(x ; \cdot))(y)=\int_{\mathbb{R}^{p}}\left(\widetilde{X}_{i_{1}} \cdots \widetilde{X}_{i_{s}} \Gamma_{\mathbb{G}}\right)\left((x, 0)^{-1} *(y, \eta)\right) \mathrm{d} \eta \\
& X_{j_{1}}^{x} \cdots X_{j_{t}}^{x}(\Gamma(\cdot ; y))(x)=\int_{\mathbb{R}^{p}}\left(\widetilde{X}_{j_{1}} \cdots \widetilde{X}_{j_{t}} \Gamma_{\mathbb{G}}\right)\left((y, 0)^{-1} *(x, \eta)\right) \mathrm{d} \eta \\
& X_{j_{1}}^{x} \cdots X_{j_{t}}^{x} X_{i_{1}}^{y} \cdots X_{i_{s}}^{y} \Gamma(x ; y) \\
& \quad=\int_{\mathbb{R}^{p}}\left(\widetilde{X}_{j_{1}} \cdots \widetilde{X}_{j_{t}}\left(\left(\widetilde{X}_{i_{1}} \cdots \widetilde{X}_{i_{s}} \Gamma_{\mathbb{G}}\right) \circ \iota\right)\right)\left((y, 0)^{-1} *(x, \eta)\right) \mathrm{d} \eta .
\end{aligned}
$$

Here $\iota$ denotes the inversion map on the Lie group $\mathbb{G} ;$ moreover, $\widetilde{X}_{1}, \ldots, \widetilde{X}_{m}$ are lifting vector fields of $X_{1}, \ldots, X_{m}$ as in Theorem $A$. 
Whereas (4.5) follows from (4.4) and from the symmetry of $\Gamma$, the representation (4.6) of the mixed derivatives is more delicate and it requires a suitable change of variable argument. This extra work is motivated by the investigation on singular integrals carried out in Section 8 .

Proof. We split the proof in four parts: (I) contains a general argument in order to pass one vector field $Z$ (indifferently operating in $x$ or $y$ ) under $\int_{\mathbb{R}^{p}} g(x, y, \eta) \mathrm{d} \eta$, for a suitable homogeneous $g$; next (II)-to-(IV) contain the proofs of (4.4)-to-(4.6).

(I) Let us consider the following families of dilations:

$$
\begin{aligned}
& D_{\lambda}(x, \xi)=\left(\delta_{\lambda}(x), E_{\lambda}(\xi)\right) \quad \text { on } \mathbb{R}_{x}^{n} \times \mathbb{R}_{\xi}^{p} \text { as in (4.16); } \\
& F_{\lambda}(x, y, \eta):=\left(\delta_{\lambda}(x), \delta_{\lambda}(y), E_{\lambda}(\eta)\right) \quad \text { on } \mathbb{R}_{x}^{n} \times \mathbb{R}_{y}^{n} \times \mathbb{R}_{\eta}^{p} ; \\
& G_{\lambda}(x, y):=\left(\delta_{\lambda}(x), \delta_{\lambda}(y)\right) \quad \text { on } \mathbb{R}_{x}^{n} \times \mathbb{R}_{y}^{n} .
\end{aligned}
$$

Let $\Omega:=\left\{(x, y, \eta) \in \mathbb{R}^{n} \times \mathbb{R}^{n} \times \mathbb{R}^{p}:(x, 0) \neq(y, \eta)\right\}$, and suppose $g \in C^{\infty}(\Omega)$ is homogeneous of degree $\alpha<q-Q$ with respect to $F_{\lambda}$. Let $Z$ be any smooth vector field in the $(x, y)$-variables, homogeneous of degree $m>0$ with respect to $G_{\lambda}$. Then, the following facts hold:

(i) for any fixed $(x, y) \in \mathbb{R}^{n} \times \mathbb{R}^{n}$ with $x \neq y$, the map $\eta \mapsto g(x, y, \eta)$ belongs to $L^{1}\left(\mathbb{R}^{p}\right)$;

(ii) $Z$ can pass under the integral sign as follows

$$
Z\left\{(x, y) \mapsto \int_{\mathbb{R}^{p}} g(x, y, \eta) \mathrm{d} \eta\right\}=\int_{\mathbb{R}^{p}} Z\{(x, y) \mapsto g(x, y, \eta)\} \mathrm{d} \eta, \quad \text { for } x \neq y .
$$

We prove (i). Let us fix $x_{0}, y_{0} \in \mathbb{R}^{n}$ such that $x_{0} \neq y_{0}$ and let $S, N$ be the homogeneous norms (with respect to $\delta_{\lambda}$ and $E_{\lambda}$, respectively)

$$
S(x):=\sum_{j=1}^{n}\left|x_{j}\right|^{1 / \sigma_{j}} \text { and } N(\eta):=\sum_{j=1}^{p}\left|\eta_{j}\right|^{1 / \tau_{j}} .
$$

Since, obviously, $\eta \mapsto g\left(x_{0}, y_{0}, \eta\right)$ belongs to $L_{\text {loc }}^{1}\left(\mathbb{R}^{p}\right)$, assertion (i) will follow if we prove that

$$
\int_{\{N>1\}} g\left(x_{0}, y_{0}, \eta\right) \mathrm{d} \eta<\infty .
$$

To this end, we first choose $\rho_{0}>0$ in such a way that $x_{0}, y_{0} \in\left\{S(x) \leq \rho_{0}\right\}$ and we observe that, since the set $K:=\left\{x: S(x) \leq \rho_{0}\right\} \times\left\{y: S(y) \leq \rho_{0}\right\} \times\{\eta: N(\eta)=1\}$ is compact and contained in $\Omega$, there exists $C>0$ such that

$$
|g(x, y, \eta)| \leq C \quad \text { for every }(x, y, \eta) \in K .
$$

On the other hand, if $\eta \in \mathbb{R}^{p}$ is such that $N(\eta)>1$ and if we set $\lambda:=1 / N(\eta) \in(0,1)$, it is readily seen that $F_{\lambda}\left(x_{0}, y_{0}, \eta\right) \in K$; thus, by (4.10) and the $F_{\lambda}$-homogeneity of $g$, we get

$$
\left|g\left(x_{0}, y_{0}, \eta\right)\right| \leq C N(\eta)^{\alpha} \quad \text { for every } \eta \in \mathbb{R}^{p} \text { with } N(\eta)>1 .
$$

Since $\alpha<q-Q$, we conclude that $\eta \mapsto g\left(x_{0}, y_{0}, \eta\right)$ is integrable on $\{N>1\}$, as a simple homogeneity argument shows (see e.g., [10, eq. (5.14)]); here one also exploits the fact that the $E_{\lambda}$-homogeneous dimension is $\sum_{j=1}^{p} \tau_{j}=Q-q$.

We prove (ii). We show that, if $Z$ is as above, then $\Phi(x, y, \eta):=Z\{(x, y) \mapsto g(x, y, \eta)\}$ is $\eta$-integrable in $\mathbb{R}^{p}$ (for any $x \neq y$ in $\mathbb{R}^{n}$ ). To this end we observe that, if we think of $Z$ as a vector field defined on $\mathbb{R}_{x}^{n} \times \mathbb{R}_{y}^{n} \times \mathbb{R}_{\eta}^{p}$ but acting only in the $(x, y)$ variables (and not on $\eta$ ), then $Z$ is $F_{\lambda}$-homogeneous of degree $m$; as a consequence, $\Phi$ is $F_{\lambda}$-homogeneous of degree $\alpha-m$. Since, by assumption, $m>0$ and $\alpha<q-Q$, we derive from statement (i) that $\Phi(x, y, \cdot)$ belongs to $L^{1}\left(\mathbb{R}^{p}\right)$ for every $x \neq y$ in $\mathbb{R}^{n}$. We now prove (4.8) with a dominated-convergence argument.

To this aim, we write

$$
\int_{\mathbb{R}^{p}}|\Phi(x, y, \eta)| \mathrm{d} \eta=\int_{\{N(\eta) \leq 1\}}|\Phi(x, y, \eta)| \mathrm{d} \eta+\int_{\{N(\eta)>1\}}|\Phi(x, y, \eta)| \mathrm{d} \eta .
$$

We fix $x_{0} \neq y_{0}$ in $\mathbb{R}^{n}$ and we provide integrable dominant functions for both the above integrals, independent of $(x, y)$ near $\left(x_{0}, y_{0}\right)$. As for the first integral, we choose $r>0$ in such a way that 
$\overline{B\left(x_{0}, r\right)} \cap \overline{B\left(y_{0}, r\right)}=\varnothing$ and we set $K:=\overline{B\left(x_{0}, r\right)} \times \overline{B\left(y_{0}, r\right)} \times\{N \leq 1\}$. By the choice of $r$, we see that $K$ is a compact subset of $\Omega$; thus, there exists $C>0$ such that

$$
|\Phi(x, y, \eta)| \leq C \text { for every }(x, y, \eta) \in K \text {. }
$$

As for the second integral, we argue as in the proof of the previous statement (i): if $\rho_{0}>0$ is such that $x_{0}, y_{0} \in\left\{S(x) \leq \rho_{0}\right\}$, from the $F_{\lambda}$-homogeneity of $\Phi$ we infer the existence of $C^{\prime}>0$ such that

$$
|\Phi(x, y, \eta)| \leq C^{\prime} N(\eta)^{\alpha-m} \quad \text { for every } x, y \in\left\{S \leq \rho_{0}\right\} \text { and every } \eta \in\{N>1\} ;
$$

since $\alpha-m<\alpha<q-Q$, the function $N^{\alpha-m}$ is integrable on $\{N>1\}$.

(II) We prove (4.4): for any $s \geq 1$, a repeated application of (I) shows that

$$
X_{i_{1}}^{y} \cdots X_{i_{s}}^{y}(\Gamma(x ; \cdot))(y)=\int_{\mathbb{R}^{p}} X_{i_{1}}^{y} \cdots X_{i_{s}}^{y}\left\{y \mapsto \Gamma_{\mathbb{G}}\left((x, 0)^{-1} *(y, \eta)\right)\right\} \mathrm{d} \eta \quad(\text { for } x \neq y) .
$$

We claim that in the above right-hand side it is legitimate to replace the vector fields $X_{i}^{y}$ with their lifted $\widetilde{X}_{i}^{(y, \eta)}$. Indeed, this will follow upon an inductive argument based on the next fact: if $h$ is smooth on $\mathbb{R}^{N} \backslash\{0\}$, and it is $D_{\lambda}$-homogeneous of degree $<q-Q$, then

$$
\int_{\mathbb{R}^{p}} X_{i}^{y}\left\{y \mapsto h\left((x, 0)^{-1} *(y, \eta)\right)\right\} \mathrm{d} \eta=\int_{\mathbb{R}^{p}}\left(\widetilde{X}_{i} h\right)\left((x, 0)^{-1} *(y, \eta)\right) \mathrm{d} \eta
$$

This will inductively prove (4.11), since $\Gamma_{\mathbb{G}}, \widetilde{X}_{i_{1}} \Gamma_{\mathbb{G}}, \widetilde{X}_{i_{1}} \widetilde{X}_{i_{2}} \Gamma_{\mathbb{G}}, \ldots$ are smooth out of 0 and $D_{\lambda}$-homogeneous of degrees $2-Q, 1-Q,-Q, \ldots$, respectively. Thus, we turn to show (4.12). To this end, by $(\mathrm{I})$, both integrals in (4.12) are finite. Moreover, we remind that

$$
\widetilde{X}_{i}^{(y, \eta)}=X_{i}^{y}+R_{i}, \quad \text { with } R_{i}=\sum_{j=1}^{p} \alpha_{i, j}(y, \eta) \frac{\partial}{\partial \eta_{j}}
$$

where $\alpha_{i, j}$ is smooth and $D_{\lambda}$-homogeneous of degree $\tau_{j}-1$; in particular $\alpha_{i, j}$ does not depend on $\eta_{j}$. Now, by exploiting the left-invariance of $\widetilde{X}_{i}$ on $\mathbb{G}=\left(\mathbb{R}^{N}, *\right)$ we have

$$
\begin{aligned}
& \int_{\mathbb{R}^{p}} X_{i}^{y}\left\{y \mapsto h\left((x, 0)^{-1} *(y, \eta)\right)\right\} \mathrm{d} \eta=\int_{\mathbb{R}^{p}}\left(\widetilde{X}_{i}^{(y, \eta)}-R_{i}\right)\left\{(y, \eta) \mapsto h\left((x, 0)^{-1} *(y, \eta)\right)\right\} \mathrm{d} \eta \\
& =\int_{\mathbb{R}^{p}} \tilde{X}_{i}^{(y, \eta)}\left\{(y, \eta) \mapsto h\left((x, 0)^{-1} *(y, \eta)\right)\right\} \mathrm{d} \eta-\int_{\mathbb{R}^{p}} R_{i}\left\{\eta \mapsto h\left((x, 0)^{-1} *(y, \eta)\right)\right\} \mathrm{d} \eta \\
& =\int_{\mathbb{R}^{p}}\left(\widetilde{X}_{i} h\right)\left((x, 0)^{-1} *(y, \eta)\right) \mathrm{d} \eta-\int_{\mathbb{R}^{p}} R_{i}\left\{\eta \mapsto h\left((x, 0)^{-1} *(y, \eta)\right)\right\} \mathrm{d} \eta
\end{aligned}
$$

This will imply (4.12) once we prove that the second integral in the far right-hand side is null. The last statement is a consequence of the following computation:

$$
\begin{aligned}
& \int_{\mathbb{R}^{p}} R_{i}\left\{\eta \mapsto h\left((x, 0)^{-1} *(y, \eta)\right)\right\} \mathrm{d} \eta=\sum_{j=1}^{p} \int_{\mathbb{R}^{p}} \alpha_{i, j}(y, \eta) \frac{\partial}{\partial \eta_{j}}\left\{h\left((x, 0)^{-1} *(y, \eta)\right)\right\} \mathrm{d} \eta \\
& =\sum_{j=1}^{p} \int_{\mathbb{R}^{p}} \frac{\partial}{\partial \eta_{j}}\left\{\alpha_{i, j}(y, \eta) h\left((x, 0)^{-1} *(y, \eta)\right)\right\} \mathrm{d} \eta=0 ;
\end{aligned}
$$

the second equality is a consequence of the fact that $\alpha_{i, j}$ does not depend on $\eta_{j}$, while the last equality derives from

$$
\lim _{\eta_{j} \rightarrow \pm \infty} \alpha_{i, j}(y, \eta) h\left((x, 0)^{-1} *(y, \eta)\right)=\alpha_{i, j}(y, \eta) \lim _{\eta_{j} \rightarrow \pm \infty} h\left((x, 0)^{-1} *(y, \eta)\right)=0 .
$$

The latter fact follows from $\lim _{|z| \rightarrow \infty} h(z)=0$ (a consequence of the $D_{\lambda}$-homogeneity of $h$ of negative degree), and since $(x, 0)^{-1} *(y, \eta)$ goes to infinity as $\left|\eta_{j}\right|$ diverges.

(III) We prove (4.5). First, since $\Gamma$ is symmetric (see Theorem A) we have

$$
\Gamma(x, y)=\int_{\mathbb{R}^{p}} \Gamma_{\mathbb{G}}\left((x, 0)^{-1} *(y, \eta)\right) \mathrm{d} \eta=\int_{\mathbb{R}^{p}} \Gamma_{\mathbb{G}}\left((y, 0)^{-1} *(x, \eta)\right) \mathrm{d} \eta .
$$


By virtue of this last representation of $\Gamma$, we can argue exactly as in Step II: for every choice of indexes $j_{1}, \ldots, j_{t} \in\{1, \ldots, m\}$ one has

$$
X_{j_{1}}^{x} \cdots X_{j_{t}}^{x}(\Gamma(\cdot ; y))(x)=\int_{\mathbb{R}^{p}}\left(\widetilde{X}_{j_{1}} \cdots \widetilde{X}_{j_{t}} \Gamma_{\mathbb{G}}\right)\left((y, 0)^{-1} *(x, \eta)\right) \mathrm{d} \eta
$$

(IV) We finally prove (4.6). By Step II, for any $i_{1}, \ldots, i_{s} \in\{1, \ldots, m\}$ one gets

$$
X_{i_{1}}^{y} \cdots X_{i_{s}}^{y}\{y \mapsto \Gamma(x ; y)\}=\int_{\mathbb{R}^{p}}\left(\widetilde{X}_{i_{1}} \cdots \widetilde{X}_{i_{s}} \Gamma_{\mathbb{G}}\right)\left((x, 0)^{-1} *(y, \eta)\right) \mathrm{d} \eta ;
$$

then, we apply the change of variable $\eta=\Phi_{x, y}(\zeta)$ in Lemma 4.5, obtaining (by (4.18))

$$
\begin{aligned}
X_{i_{1}}^{y} \cdots X_{i_{s}}^{y}\{y \mapsto \Gamma(x ; y)\} & =\int_{\mathbb{R}^{p}}\left(\widetilde{X}_{i_{1}} \cdots \widetilde{X}_{i_{s}} \Gamma_{\mathbb{G}}\right)\left((x, \zeta)^{-1} *(y, 0)\right) \mathrm{d} \zeta \\
& =\int_{\mathbb{R}^{p}}\left(\left(\widetilde{X}_{i_{1}} \cdots \widetilde{X}_{i_{s}} \Gamma_{\mathbb{G}}\right) \circ \iota\right)\left((y, 0)^{-1} *(x, \zeta)\right) \mathrm{d} \zeta .
\end{aligned}
$$

On account of an analogous $x$-derivative formulation of (4.12) (with $\left.h=\left(\widetilde{X}_{i_{1}} \cdots \widetilde{X}_{i_{s}} \Gamma_{\mathbb{G}}\right) \circ \iota\right)$, for every choice of $j_{1}, \ldots, j_{t} \in\{1, \ldots, m\}$ we obtain

$$
X_{j_{1}}^{x} \cdots X_{j_{t}}^{x} X_{i_{1}}^{y} \cdots X_{i_{s}}^{y} \Gamma(x ; y)=\int_{\mathbb{R}^{p}}\left(\widetilde{X}_{j_{1}} \cdots \widetilde{X}_{j_{t}}\left(\left(\widetilde{X}_{i_{1}} \cdots \widetilde{X}_{i_{s}} \Gamma_{\mathbb{G}}\right) \circ \iota\right)\right)\left((y, 0)^{-1} *(x, \zeta)\right) \mathrm{d} \zeta .
$$

This is precisely (4.6), and the proof is complete.

In the previous proof we used the technical Lemma 4.5 concerning the operation $*$. First we need to closely scrutinize the construction of the lifting group in Theorem A:

Remark 4.4. In the sequel, we shall need to invoke the explicit construction of the group $\mathbb{G}$ in point (1) of Theorem A, which is now in order; for all the details, see [10].

First of all, since $X_{1}, \ldots, X_{m}$ are $\delta_{\lambda}$-homogeneous of degree 1 , then $\mathfrak{a}:=\operatorname{Lie}(X)$ is nilpotent; as a consequence, if $\diamond$ denotes the Baker-Campbell-Hausdorff series on $\mathfrak{a}$ (boiling down to a finite sum), it is well known that $(\mathfrak{a}, \diamond)$ is a Lie group (whose inversion map is $X \mapsto-X$ ). Moreover, as $X_{1}, \ldots, X_{m}$ are linearly independent in $\mathfrak{a}$, we can choose a basis for $\mathfrak{a}$ as

$$
\mathcal{E}=\left\{X_{1}, \ldots, X_{m}, X_{m+1}, \ldots, X_{N}\right\} .
$$

We can equip $\mathbb{R}^{N}$ with a Lie group structure $\left(\mathbb{R}^{N}, \bullet\right)$ by reading $\diamond$ in $\mathcal{E}$-coordinates, i.e.,

$$
\sum_{j=1}^{N}(a \bullet b)_{j} X_{j}=\left(\sum_{j=1}^{N} a_{j} X_{j}\right) \diamond\left(\sum_{j=1}^{N} b_{j} X_{j}\right), \quad \text { for every } a, b \in \mathbb{R}^{N} .
$$

For a future use, we set $a \cdot X:=\sum_{j=1}^{N} a_{j} X_{j}$ for any $a \in \mathbb{R}^{N}$. Folland [20] proved that the map

$$
\Pi: \mathbb{R}^{N} \longrightarrow \mathbb{R}^{n}, \quad \Pi(a):=\Phi_{1}^{a \cdot X}(0)
$$

is surjective; here, given a vector field $Y \in \mathfrak{a}$, we denote by $\Phi_{t}^{Y}(z)$ the flow of $Y$ at time $t$ starting from $z$ at $t=0$. In [10] it is proved that there always exist indexes $j_{1}, \ldots, j_{p} \in\{1, \ldots, N\}$ such that

$$
T: \mathbb{R}^{N} \longrightarrow \mathbb{R}^{N}, \quad T(a):=\left(\Pi(a), a_{j_{1}}, \ldots, a_{j_{p}}\right)
$$

is a smooth diffeomorphism. Finally, the group $\mathbb{G}=\left(\mathbb{R}^{N}, *\right)$ is obtained as follows:

$$
z * z^{\prime}:=T\left(T^{-1}(z) \bullet T^{-1}\left(z^{\prime}\right)\right), \quad \text { for every } z, z^{\prime} \in \mathbb{R}^{N} .
$$

It can also be proved that $\mathbb{G}$ is a homogeneous group with dilations

$$
D_{\lambda}: \mathbb{R}^{N}=\mathbb{R}^{n} \times \mathbb{R}^{p} \longrightarrow \mathbb{R}^{N}, \quad D_{\lambda}(x, \xi)=\left(\delta_{\lambda}(x), E_{\lambda}(\xi)\right),
$$

where $E_{\lambda}(\xi)=\left(\lambda^{\tau_{1}} \xi, \ldots, \lambda^{\tau_{p}} \xi_{p}\right)$ for suitable integers $1 \leq \tau_{1} \leq \cdots \leq \tau_{p}$. We point out that the exponents of $D_{\lambda}$ are not increasingly ordered (but this is true of the $\sigma_{i}$ 's and of $\tau_{j}$ 's separately). This will cause some subtleties in handling the group structure of $\mathbb{G}$ (see, e.g., Lemma 4.5).

The proof of the next lemma is quite delicate, due to the lack of ordering of the exponents of the dilation $D_{\lambda}$ of $\mathbb{G}$. 
Lemma 4.5. For every $x, y \in \mathbb{R}^{n}$ and every $\eta \in \mathbb{R}^{p}$, we set

$$
\theta(x, y, \eta):=(x, 0) *(x, \eta)^{-1} *(y, 0) .
$$

Denoting by $\pi$ the projection of $\mathbb{R}^{n} \times \mathbb{R}^{p}$ onto $\mathbb{R}^{p}$, the map

$$
\eta \mapsto \Phi_{x, y}(\eta):=\pi(\theta(x, y, \eta))
$$

is a smooth diffeomorphism of $\mathbb{R}^{p}$ (for any fixed $x, y \in \mathbb{R}^{n}$ ) whose Jacobian determinant is \pm 1 . Furthermore, the following identity holds:

$$
(x, 0)^{-1} *\left(y, \Phi_{x, y}(\zeta)\right)=(x, \zeta)^{-1} *(y, 0), \quad \text { for every } x, y \in \mathbb{R}^{n} \text { and every } \zeta \in \mathbb{R}^{p} .
$$

In particular, $y_{1}, \ldots, y_{n}$ are the first $n$ components of $\theta(x, y, \eta)$.

In other words, the change of variable

$$
\eta=\Phi_{x, y}(\zeta)
$$

satisfies $\mathrm{d} \eta=\mathrm{d} \zeta$ and

$$
(x, 0)^{-1} *(y, \eta)=(x, \zeta)^{-1} *(y, 0) .
$$

Proof. We follow the notation in Remark 4.4. As observed at the end of that remark, the last $p$ exponents of the dilation $D_{\lambda}$ of $\mathbb{G}$ are increasingly ordered; as a consequence, arguing as in [13, Theorem 1.3.15], it is not difficult to check that (for every $k=1, \ldots, p$ )

$$
\theta_{n+k}(x, y, \eta)=\sum_{j: \tau_{j}=\tau_{k}} c_{j} \eta_{j}+Q_{k}(x, y, \eta),
$$

where the $c_{j}$ 's are real numbers and $Q_{k}$ is a polynomial only depending the $\eta_{j}$ 's such that $\tau_{j}<\tau_{k}$. Since $\theta(0,0, \eta)=(0, \eta)^{-1}$ (whose components are $E_{\lambda}$-homogeneous functions), one can reproduce the arguments in [13, Corollary 1.3.16] and infer that

$$
\theta_{k}(0,0, \eta)=-\eta_{k}+q_{k}(\eta)
$$

where $q_{k}$ is a polynomial only depending the $\eta_{j}$ 's such that $\tau_{j}<\tau_{k}$. By gathering together (4.19) and (4.20) we obtain

$$
\theta_{n+k}(x, y, \eta)=-\eta_{k}+Q_{k}(x, y, \eta)
$$

which readily proves that $\Phi_{x, y}$ is a polynomial diffeomorphism whose Jacobian determinant is \pm 1 .

We now prove (4.17), which is equivalent to

$$
\left(y, \Phi_{x, y}(\zeta)\right)=(x, 0) *(x, \zeta)^{-1} *(y, 0)=\theta(x, y, \zeta) .
$$

On account of the very definition of $\Phi_{x, y}$, this last identity is will follow if we prove that $y_{1}, \ldots, y_{n}$ are the first $n$ components of $\theta(x, y, \zeta)$. We now invoke the explicit construction of the operation $*$ in Remark 4.4, whose notation we fully inherit. In this notation, we have

$$
\theta(x, y, \zeta)=T\left(T^{-1}(x, 0) \bullet T^{-1}\left((x, \zeta)^{-1}\right) \bullet T^{-1}(y, 0)\right) .
$$

As a consequence, we need to prove that (see the notation in (4.15) )

$$
\Pi\left(T^{-1}(x, 0) \bullet T^{-1}\left((x, \zeta)^{-1}\right) \bullet T^{-1}(y, 0)\right)=y .
$$

Let then $a, b, c \in \mathbb{R}^{N}$ be such that $T(a)=(x, 0), T(b)=(x, \zeta)$ and $T(c)=(y, 0)$. In particular, we have $\Pi(a)=\Pi(b)=x$ and $\Pi(c)=y$. Since, by definition, $T$ is a homomorphism of $\left(\mathbb{R}^{N}, \bullet\right)$ onto $\left(\mathbb{R}^{N}, *\right)$, and since the inversion map of $\left(\mathbb{R}^{N}, \bullet\right)$ is $z \mapsto-z$ (see Remark 4.4), we can write

$$
T^{-1}(x, 0) \bullet T^{-1}\left((x, \zeta)^{-1}\right) \bullet T^{-1}(y, 0)=a \bullet(-b) \bullet c ;
$$

as a consequence, by the very definitions of $\bullet$ and $\Pi$ (see (4.13) and (4.14)), we have

$$
\Pi(a \bullet(-b) \bullet c)=\Phi_{1}^{(a \bullet(-b) \bullet c) \cdot X}(0)=\Phi_{1}^{(a \cdot X) \diamond(-b \cdot X) \diamond(c \cdot X)}(0) .
$$

By exploiting the Baker-Campbell-Hausdorff Theorem for ODEs (see [11, Chapter 13]) we obtain

$$
\Phi_{1}^{(a \cdot X) \diamond(-b \cdot X) \diamond(c \cdot X)}(0)=\Phi_{1}^{c \cdot X}\left(\Phi_{1}^{-b \cdot X}\left(\Phi_{1}^{a \cdot X}(0)\right)\right)=\Phi_{1}^{c \cdot X}\left(\Phi_{1}^{-b \cdot X}(x)\right)=\Phi_{1}^{c \cdot X}(0)=y .
$$

The second equality follows from $\Pi(a)=x$; the third equality is a consequence of $\Phi_{1}^{b \cdot X}(0)=x$ together with the semigroup property $\left(\Phi_{t}^{Y}\right)^{-1}=\Phi_{t}^{-Y}$; the last equality follows from $\Pi(c)=y$. 
From now on, we adopt our abused notation $d_{\widetilde{X}}\left(z, z^{\prime}\right)=d_{\widetilde{X}}\left(z^{-1} * z^{\prime}\right)$.

Proposition 4.6. For every integer $r \geq 0$ there exists $c>0$ (depending on $r$ and on the set $X$ ) such that, for every $x, y \in \mathbb{R}^{n}$ (with $\left.x \neq y\right)$, one has

$$
\left|Z_{1} \cdots Z_{r} \Gamma(x ; y)\right| \leq c \int_{\mathbb{R}^{p}} d_{\widetilde{X}}^{2-Q-r}\left((x, 0)^{-1} *(y, \eta)\right) \mathrm{d} \eta,
$$

for any choice of $Z_{1}, \ldots, Z_{r}$ as in (4.2). Here, we remind that $Q$ is the homogeneous dimension of the Carnot group $\mathbb{G}=\left(\mathbb{R}^{N}, *, D_{\lambda}\right)$. In particular, the map

$$
\eta \mapsto d_{\widetilde{X}}^{2-Q-r}\left((x, 0)^{-1} *(y, \eta)\right)
$$

belongs to $L^{1}\left(\mathbb{R}^{p}\right)$ for every $x \neq y \in \mathbb{R}^{n}$ and every $r \geq 0$.

Proof. When $r=0$ (so that no derivatives apply), (4.21) is a simple consequence of (1.7) and

$$
c^{-1} d_{\widetilde{X}}(0, z)^{2-Q} \leq \Gamma_{\mathbb{G}}(z) \leq c d_{\widetilde{X}}(0, z)^{2-Q}, \quad \forall z \in \mathbb{R}^{N} \backslash\{0\},
$$

where $c \geq 1$ is a constant only depending on the group $\mathbb{G}$ and the set $\widetilde{X}=\left\{\widetilde{X}_{1}, \ldots, \widetilde{X}_{m}\right\}$; the latter estimate trivially follows from the fact that $\Gamma_{\mathbb{G}}(z)^{1 /(2-Q)}$ and $d_{\widetilde{X}}(0, z)$ are homogeneous norms on $\mathbb{G}$, and the equivalence of all homogeneous norms on $\mathbb{G}$ (see e.g., [13, Prop. 5.1.4]).

When $r \geq 1$, a repeated use of the map $\iota$ in (4.5)-4.6) will allow us to express the therein integrands as functions of $(x, 0)^{-1} *(y, \eta)$ : this will lead to a unitary proof of (4.1). Indeed, we claim that (4.5)-(4.6) can be rewritten as follows:

$$
\begin{aligned}
& X_{j_{1}}^{x} \cdots X_{j_{t}}^{x}(\Gamma(\cdot ; y))(x)=\int_{\mathbb{R}^{p}}\left(\left(\widetilde{X}_{j_{1}} \cdots \widetilde{X}_{j_{t}} \Gamma_{\mathbb{G}}\right) \circ \iota\right)\left((x, 0)^{-1} *(y, \eta)\right) \mathrm{d} \eta ; \\
& X_{j_{1}}^{x} \cdots X_{j_{t}}^{x} X_{i_{1}}^{y} \cdots X_{i_{s}}^{y} \Gamma(x ; y) \\
& \quad=\int_{\mathbb{R}^{p}}\left\{\left(\widetilde{X}_{j_{1}} \cdots \widetilde{X}_{j_{t}}\left(\left(\widetilde{X}_{i_{1}} \cdots \widetilde{X}_{i_{s}} \Gamma_{\mathbb{G}}\right) \circ \iota\right)\right) \circ \iota\right\}\left((x, 0)^{-1} *(y, \eta)\right) \mathrm{d} \eta .
\end{aligned}
$$

As for (4.23), it suffices to apply to (4.5) the change of variable $\eta=\Phi_{y, x}(\zeta)$ in Lemma 4.5, together with (4.18) (with $x, y$ interchanged). As for (4.24), we start from (4.6) and we argue as above (plus another insertion of the $\iota$ map).

We now exploit (4.4) (with $s=r$ ), (4.23) (with $t=r$ ) and (4.24) (with $s=r_{1}, t=r_{2}$ and $\left.r_{1}+r_{2}=r\right)$ : in all three cases, the functions

$$
\tilde{X}_{i_{1}} \cdots \widetilde{X}_{i_{s}} \Gamma_{\mathbb{G}}, \quad\left(\widetilde{X}_{j_{1}} \cdots \tilde{X}_{j_{t}} \Gamma_{\mathbb{G}}\right) \circ \iota, \quad\left(\tilde{X}_{j_{1}} \cdots \tilde{X}_{j_{r_{1}}}\left(\left(\widetilde{X}_{i_{1}} \cdots \tilde{X}_{i_{r_{2}}} \Gamma_{\mathbb{G}}\right) \circ \iota\right)\right) \circ \iota
$$

are smooth out of the origin of $\mathbb{R}^{N}$, and $D_{\lambda}$-homogeneous of degree $2-Q-r$; thus, for simple homogeneity arguments, their absolute values are bounded from above by $c d_{\widetilde{X}}^{2-Q-r}(0, \cdot)$, where $c>0$ is a constant only depending on $r$ (and the system $X$ ). This readily gives (4.21).

As for the last statement of the proposition, it follows from (I) in the proof of Lemma 4.3. taking into account that $2-Q-r<q-Q($ as $q>2)$.

On account of Proposition 4.6, the next step towards the proof of Theorem 4.1 is to estimate the integral in the right-hand side of (4.21). This is accomplished as follows:

- the estimate of the integral for arbitrary $x, y$ will follow by a homogeneity argument as in Remark 3.1, once it is proved for $x, y$ in some fixed compact neighborhood $K$ of $0 \in \mathbb{R}^{n}$;

- for $x \neq y \in K$, the right-hand side of (4.21) will be estimated by splitting the integral in the two parts $\{|\eta| \geq \delta\}$ and $\{|\eta|<\delta\}$, where $\delta>0$ is some fixed positive number.

The two needed estimates are provided in the following Propositions 4.7 and 4.8, which we now state.

Proposition 4.7. For every compact neighborhood of the origin, say $K \subset \mathbb{R}^{n}$, for every integer $r \geq 0$ and every $\delta>0$, there exists $C_{1}=C_{1}(K, r, \delta)>0$ such that

$$
\int_{|\eta| \geq \delta} d_{\widetilde{X}}^{2-Q-r}\left((x, 0)^{-1} *(y, \eta)\right) \mathrm{d} \eta \leq C_{1} \frac{d_{X}(x, y)^{2-r}}{\left|B_{X}\left(x, d_{X}(x, y)\right)\right|},
$$

for every $x, y \in K$ with $x \neq y$. 
Proposition 4.8. For every compact neighborhood of the origin, say $K \subset \mathbb{R}^{n}$, for every integer $r \geq 0$ and every $\delta>0$, there exists $C_{2}=C_{2}(r)>0$ such that

$$
\int_{|\eta|<\delta} d_{\widetilde{X}}^{2-Q-r}\left((x, 0)^{-1} *(y, \eta)\right) \mathrm{d} \eta \leq C_{2} \frac{d_{X}(x, y)^{2-r}}{\left|B_{X}\left(x, d_{X}(x, y)\right)\right|},
$$

for every $x, y \in K$ with $x \neq y$.

With the above propositions at hand (whose proofs will be shortly provided), we can give the

Proof of Theorem 4.1. Let the notation of Theorem 4.1 be understood; we arbitrarily fix a compact neighborhood $K$ of the origin in $\mathbb{R}^{n}$ and a number $\delta>0$. Fixing $x \neq y \in K$, we have the following estimate, based on Propositions 4.6, 4.7 and 4.8.

$$
\begin{aligned}
& \left|Z_{1} \cdots Z_{r} \Gamma(x ; y)\right| \stackrel{\text { 4.21 }}{\leq} c \int_{\mathbb{R}^{p}} d_{\widetilde{X}}^{2-Q-r}\left((x, 0)^{-1} *(y, \eta)\right) \mathrm{d} \eta \\
& =c \int_{|\eta| \geq \delta} d_{\widetilde{X}}^{2-Q-r}\left((x, 0)^{-1} *(y, \eta)\right) \mathrm{d} \eta+c \int_{|\eta|<\delta} d_{\widetilde{X}}^{2-Q-r}\left((x, 0)^{-1} *(y, \eta)\right) \mathrm{d} \eta
\end{aligned}
$$

(we use (4.25) and (4.26), and we set $C:=c(r) \max \left\{C_{1}(K, r, \delta), C_{2}(r)\right\}$ )

$$
\leq C \frac{d_{X}(x, y)^{2-r}}{\left|B_{X}\left(x, d_{X}(x, y)\right)\right|}
$$

We remove the condition $x, y \in K$ by a homogeneity argument. It suffices to apply Remark 3.1 to

$$
F(x, y):=\left|Z_{1} \cdots Z_{r} \Gamma(x ; y)\right| \quad \text { and } \quad G(x, y):=C \frac{d_{X}(x, y)^{2-r}}{\left|B_{X}\left(x, d_{X}(x, y)\right)\right|},
$$

with the choices (see the notation in Remark 3.1) $m=2 n, \Omega=\left\{(x, y) \in \mathbb{R}^{2 n}: x \neq y\right\}$ and the dilation $M_{\lambda}$ given by $G_{\lambda}$ in (4.7). We can apply the cited lemma, as $F$ and $G$ are both $M_{\lambda^{-}}$ homogeneous of degree $2-q-r$ (a simple consequence of (1.8), (2.2) and the $\delta_{\lambda}$-homogeneity of $\left.Z_{1}, \ldots, Z_{r}\right)$, and since $F \leq G$ is valid on $(K \times K) \cap \Omega$ (see (4.27) $)$.

Finally, we prove the vanishing property in (4.3). To this end, it is sufficient to prove that, for any fixed $x \in \mathbb{R}^{n}$, the right-hand side of (4.1) goes to 0 as $|y| \rightarrow \infty$. This is a simple consequence of (2.7) which indeed gives

$$
\frac{d_{X}(x, y)^{2-r}}{\left|B_{X}\left(x, d_{X}(x, y)\right)\right|} \leq \frac{d_{X}(x, y)^{2-r}}{\gamma_{1} \sum_{k=n}^{q} f_{k}(x) d_{X}(x, y)^{k}} \leq \frac{1}{\gamma_{1} f_{q} d_{X}(x, y)^{q-2+r}} \longrightarrow 0 \quad \text { as }|y| \rightarrow \infty .
$$

The latter follows from $f_{q}(x)=f_{q}>0$ and $q-2+r \geq q-2>0$. This ends the proof.

We now give the proofs of Propositions 4.7 and 4.8

Proof of Proposition 4.7. Let $K, r, \delta$ be as in the statement of the proposition. We consider the open set $\Omega:=\left\{(x, y, \eta) \in \mathbb{R}^{n} \times \mathbb{R}^{n} \times \mathbb{R}^{p}:(x, 0) \neq(y, \eta)\right\}$, and we set

$$
g(x, y, \eta):=d_{\widetilde{X}}^{2-Q-r}\left((x, 0)^{-1} *(y, \eta)\right) .
$$

If $S$ and $N$ are as in (4.9), we choose $\varepsilon=\varepsilon(\delta)>0$ so small that $\{|\eta| \geq \delta\} \subseteq\{N(\eta) \geq \varepsilon\}$ and we choose $R=R(K)>0$ so large that $K \subseteq\{S(x) \leq R\}$. Since

$$
T:=\{x: S(x) \leq R\} \times\{y: S(y) \leq R\} \times\{\eta: N(\eta)=\varepsilon\}
$$

is compact and contained in $\Omega$, there exists $c=c(\varepsilon, R)>0$ such that $g\left(x^{\prime}, y^{\prime}, \eta^{\prime}\right) \leq c$ for every $\left(x^{\prime}, y^{\prime}, \eta^{\prime}\right) \in T$. Now, if $x, y \in K$ and $N(\eta) \geq \varepsilon$, choosing $\lambda=\varepsilon / N(\eta) \leq 1$ we clearly have

$$
\left(x^{\prime}, y^{\prime}, \eta^{\prime}\right):=F_{\lambda}(x, y, \eta) \in T
$$

where $F_{\lambda}$ is as in (4.7). As a consequence, since $g$ is $F_{\lambda}$-homogeneous of degree $2-Q-r$ we get

$$
g(x, y, \eta)=g\left(F_{1 / \lambda}\left(x^{\prime}, y^{\prime}, \eta^{\prime}\right)\right)=\frac{1}{\lambda^{2-Q-r}} g\left(x^{\prime}, y^{\prime}, \eta^{\prime}\right) \leq \frac{c}{\varepsilon^{2-Q-r}} N(\eta)^{2-Q-r}
$$


Summing up, we have

$$
\begin{aligned}
& \int_{|\eta| \geq \delta} d_{\widetilde{X}}^{2-Q-r}\left((x, 0)^{-1} *(y, \eta)\right) \mathrm{d} \eta \leq \int_{N(\eta) \geq \varepsilon} g(x, y, \eta) \mathrm{d} \eta \\
& \leq \frac{c}{\varepsilon^{2-Q-r}} \int_{\{N(\eta) \geq \varepsilon\}} N(\eta)^{2-Q-r} \mathrm{~d} \eta=c(\varepsilon, R) \varepsilon^{Q-q} \int_{N(\eta) \geq 1} N(\eta)^{2-Q-r} \mathrm{~d} \eta=: C(K, r, \delta) .
\end{aligned}
$$

Note that $C(K, r, \delta)$ is finite, since the function $N^{2-Q-r}$ is integrable on $\{N \geq 1\}$ (as one can readily deduce from $2-Q-r<q-Q$ ). The above estimate will give (4.25) once we prove that

$$
\inf _{\substack{x, y \in K \\ x \neq y}} \frac{d_{X}(x, y)^{2-r}}{\left|B_{X}\left(x, d_{X}(x, y)\right)\right|}>0 .
$$

In order to prove (4.28) we make use of Theorem B in the introduction: thanks to (2.7), we have

$$
\sup _{\substack{x, y \in K \\ x \neq y}} \frac{\left|B_{X}\left(x, d_{X}(x, y)\right)\right|}{d_{X}(x, y)^{2-r}} \leq \gamma_{2} \sup _{\substack{x, y \in K \\ x \neq y}}\left(\sum_{k=n}^{q} f_{k}(x) d_{X}(x, y)^{k+r-2}\right)=: M(K, r)<\infty,
$$

since the functions $f_{k}$ 's are continuous and, by assumption, $n+r-2 \geq 0$. This implies at once (4.25) with the choice $C_{1}(K, r, \delta):=C(K, r, \delta) M(K, r)$, and the proof is complete.

Proof of Proposition 4.8. It follows by combining the next Lemmas 4.9 and 4.10, with the choice $\beta=2-r$ (note that, since $r \geq 0$, we have $\beta \leq 2<n+1$ ), and $C_{2}(r):=C_{3}(2-r) C_{4}(2-r)$.

Lemma 4.9. Let $K \subset \mathbb{R}^{n}$ be any compact neighborhood of the origin, let $\delta$ be any positive real number, and finally let $\beta \leq n+1$.

Then there exist positive numbers $R_{0}=R_{0}(K, \delta)$ and $C_{3}=C_{3}(\beta)$ such that

$$
\int_{|\eta|<\delta} d_{\widetilde{X}}^{\beta-Q}\left((x, 0)^{-1} *(y, \eta)\right) \mathrm{d} \eta \leq C_{3} \int_{d_{X}(x, y)}^{R_{0}} \frac{\rho^{\beta-1}}{\left|B_{X}(x, \rho)\right|} \mathrm{d} \rho
$$

for every $x, y \in K$ with $x \neq y$. Estimate (4.30) is meaningful, since $R_{0}$ is chosen in such a way that $R_{0}>2 d_{X}(x, y)$ for all $x, y \in K$ (and the integral in the right-hand side does not vanish).

Proof. The following argument is adapted from [27, Theorem 5]. We shall use in a crucial way the estimate (2.8) (with $\xi=0$ ): there exists a structural constant $c_{1}>0$ such that

$$
\left|\left\{\eta \in \mathbb{R}^{p}:(y, \eta) \in B_{\widetilde{X}}((x, 0), \rho)\right\}\right| \leq c_{1} \frac{\left|B_{\widetilde{X}}((x, 0), \rho)\right|}{\left|B_{X}(x, \rho)\right|},
$$

for every $x, y \in \mathbb{R}^{n}$ and every $\rho>0$.

Let now $K, \delta, \beta$ be as in the statement of the lemma, and let $R_{0}=R_{0}(K, \delta)>0$ be such that

$$
d_{\widetilde{X}}\left((x, 0)^{-1} *(y, \eta)\right)<\frac{R_{0}}{2}, \quad \text { for any } x, y \in K \text { and any }|\eta| \leq \delta .
$$

Henceforth, we fix $x \neq y$ in $K$. Since we have

$$
d_{X}(x, y) \stackrel{(2.4)}{\leq} d_{\widetilde{X}}\left((x, 0)^{-1} *(y, \eta)\right) \stackrel{\sqrt[4.32]{<}}{<} \frac{R_{0}}{2}
$$

we choose the unique integer $k \geq 1$ such that

$$
\frac{R_{0}}{2^{k+1}} \leq d_{X}(x, y)<\frac{R_{0}}{2^{k}}
$$

On account of (4.32), this last estimate implies that

$$
\frac{R_{0}}{2^{k+1}} \leq d_{\widetilde{X}}\left((x, 0)^{-1} *(y, \eta)\right)<\frac{R_{0}}{2},
$$


for every $\eta \in \mathbb{R}^{p}$ with $|\eta| \leq \delta$. Thus we have the following computation:

$$
\begin{aligned}
& \int_{|\eta|<\delta} d_{\widetilde{X}}^{\beta-Q}\left((x, 0)^{-1} *(y, \eta)\right) \mathrm{d} \eta \\
& \leq \int_{\left\{\eta \in \mathbb{R}^{p}: \frac{R_{0}}{2^{k+1}} \leq d_{\widetilde{X}}\left((x, 0)^{-1} *(y, \eta)\right)<\frac{R_{0}}{2}\right\}} d_{\widetilde{X}}^{\beta-Q}\left((x, 0)^{-1} *(y, \eta)\right) \mathrm{d} \eta \\
& \quad=\sum_{j=1}^{k} \int_{\mathbb{R}^{p}} d_{\widetilde{X}}^{\beta-Q}\left((x, 0)^{-1} *(y, \eta)\right) \chi_{A_{j}}(\eta) \mathrm{d} \eta=:(\star)
\end{aligned}
$$

where we have introduced the notation

$$
A_{j}:=\left\{\eta \in \mathbb{R}^{p}: \frac{R_{0}}{2^{j+1}} \leq d_{\widetilde{X}}\left((x, 0)^{-1} *(y, \eta)\right)<\frac{R_{0}}{2^{j}}\right\} .
$$

We now observe that:

- for any $\eta \in A_{j}$ we have (see also (2.5)

$$
d_{\widetilde{X}}^{Q}\left((x, 0)^{-1} *(y, \eta)\right) \geq\left(\frac{R_{0}}{2^{j+1}}\right)^{Q}=\omega_{Q}\left|B_{\widetilde{X}}\left((x, 0), \frac{R_{0}}{2^{j+1}}\right)\right| ;
$$

- for any $\eta \in A_{j}$ one has

$$
d_{\widetilde{X}}^{\beta}\left((x, 0)^{-1} *(y, \eta)\right) \leq c_{\beta}\left(\frac{R_{0}}{2^{j}}\right)^{\beta}, \quad \text { where } \quad c_{\beta}= \begin{cases}1, & \text { if } \beta \geq 0 \\ 2^{-\beta}, & \text { if } \beta<0 .\end{cases}
$$

Gathering together these facts, one has

$$
\begin{aligned}
(\star) & \leq \frac{c_{\beta}}{\omega_{Q}} \sum_{j=1}^{k} \frac{\left(2^{-j} R_{0}\right)^{\beta}}{\left|B_{\widetilde{X}}\left((x, 0), 2^{-j-1} R_{0}\right)\right|} \int_{\mathbb{R}^{p}} \chi_{A_{j}}(\eta) \mathrm{d} \eta \\
& \leq \frac{c_{\beta}}{\omega_{Q}} \sum_{j=1}^{k} \frac{\left(2^{-j} R_{0}\right)^{\beta}}{\left|B_{\widetilde{X}}\left((x, 0), 2^{-j-1} R_{0}\right)\right|} \cdot\left|\left\{\eta \in \mathbb{R}^{p}:(y, \eta) \in B_{\widetilde{X}}\left((x, 0), 2^{-j} R_{0}\right)\right\}\right| \\
& \stackrel{(4.31)}{\leq} \frac{c_{1} c_{\beta}}{\omega_{Q}} \sum_{j=1}^{k} \frac{\left(2^{-j} R_{0}\right)^{\beta}}{\left|B_{\widetilde{X}}\left((x, 0), 2^{-j-1} R_{0}\right)\right|} \cdot \frac{\left|B_{\widetilde{X}}\left((x, 0), 2^{-j} R_{0}\right)\right|}{\left|B_{X}\left(x, 2^{-j} R_{0}\right)\right|} \\
& \stackrel{(2.5)}{=} C(\beta) \sum_{j=1}^{k} \frac{\left(2^{-j} R_{0}\right)^{\beta}}{\left|B_{X}\left(x, 2^{-j} R_{0}\right)\right|}=:(2 \star), \quad \text { with } C(\beta):=\frac{2^{Q} c_{1} c_{\beta}}{\omega_{Q}} .
\end{aligned}
$$

We now claim that, for a fixed $x$, the (continuous) function

$$
(0, \infty) \ni \rho \mapsto \frac{\rho^{\beta-1}}{\left|B_{X}(x, \rho)\right|}
$$

is comparable to a (continuous) monotone decreasing function, say $g_{x}(\rho)$. Indeed, by (2.7) we have

$$
\frac{\left|B_{X}(x, \rho)\right|}{\rho^{\beta-1}} \approx \sum_{h=n}^{q} f_{h}(x) \rho^{h+1-\beta}, \quad \text { for } \rho>0,
$$

for suitable non-negative functions $f_{h}$ 's. As a consequence, observing that the exponents of $\rho$ in (4.34) are all non-negative (as $\beta \leq n+1$ by assumption), the right-hand side of (4.34) is monotone increasing in $\rho$; this proves that

$$
g_{x}(\rho):=\left(\sum_{h=n}^{q} f_{h}(x) \rho^{h+1-\beta}\right)^{-1}
$$


is monotone decreasing, fulfilling our claim. This gives the next chain of inequalities:

$$
\begin{aligned}
(2 \star) & =C(\beta) \sum_{j=1}^{k} \frac{\left(2^{-j} R_{0}\right)^{\beta-1}}{\left|B_{X}\left(x, 2^{-j} R_{0}\right)\right|} \cdot 2^{-j} R_{0} \\
& \leq \frac{C(\beta)}{\gamma_{1}} \sum_{j=1}^{k} g_{x}\left(2^{-j} R_{0}\right) \cdot 2^{-j} R_{0}=\frac{2 C(\beta)}{\gamma_{1}} \sum_{j=1}^{k} g_{x}\left(2^{-j} R_{0}\right) \cdot 2^{-j-1} R_{0} \\
& \leq \frac{2 C(\beta)}{\gamma_{1}} \sum_{j=1}^{k} \int_{2^{-j-1} R_{0}}^{2^{-j} R_{0}} g_{x}(\rho) \mathrm{d} \rho=\frac{2 C(\beta)}{\gamma_{1}} \int_{2^{-k-1} R_{0}}^{2^{-1} R_{0}} g_{x}(\rho) \mathrm{d} \rho \\
& \leq \frac{2 \gamma_{2} C(\beta)}{\gamma_{1}} \int_{2^{-k-1} R_{0}}^{2^{-1} R_{0}} \frac{\rho^{\beta-1}}{\left|B_{X}(x, \rho)\right|} \mathrm{d} \rho=\frac{\gamma_{2} C(\beta)}{2^{\beta-1} \gamma_{1}} \int_{2^{-k} R_{0}}^{R_{0}} \frac{t^{\beta-1}}{\left|B_{X}(x, t / 2)\right|} \mathrm{d} t \\
& \leq C_{3}(\beta) \int_{d_{X}(x, y)}^{R_{0}} \frac{t^{\beta-1}}{\left|B_{X}(x, t)\right|} \mathrm{d} t, \quad \text { with } C_{3}(\beta):=\frac{C_{d} \gamma_{2} C(\beta)}{2^{\beta-1} \gamma_{1}} .
\end{aligned}
$$

In the last inequality we used the positivity of $g_{x}(\rho)$ and (4.33), jointly with the doubling inequality (2.3). This ends the proof.

Finally, the following lemma is proved in [16, Lemma 3.1], and we provide the proof for completeness.

Lemma 4.10. For every $\beta \in \mathbb{R}$ there exists a constant $C_{4}=C_{4}(\beta)>0$ such that, for every $0<a<b$ and every $x \in \mathbb{R}^{n}$, the following inequalities hold:

$$
\int_{a}^{b} \frac{\rho^{\beta-1}}{\left|B_{X}(x, \rho)\right|} \mathrm{d} \rho \leq \begin{cases}C_{4}(\beta) \frac{a^{\beta}}{\left|B_{X}(x, a)\right|} & \text { for } \beta<n, \\ C_{4}(\beta) \frac{a^{n}}{\left|B_{X}(x, a)\right|} \log \left(\frac{b}{a}\right) & \text { for } \beta=n, \\ C_{4}(\beta) \frac{a^{n}}{\left|B_{X}(x, a)\right|} b^{\beta-n} & \text { for } \beta>n .\end{cases}
$$

We shall apply this lemma with the choice $a=d_{X}(x, y)$ (with $\left.x \neq y\right)$ and $b=R_{0}>d_{X}(x, y)$. Moreover, in order to prove Theorem 4.1, we shall take $\beta=2-r$ (with $r \geq 0$ ) which falls in the first estimate in (4.35), since $2-r \leq 2<n$ in our case.

Proof. Starting from (2.7), one can obtain the following estimate (see also [16, Remark 2.12]):

$$
\left|B_{X}(x, \rho)\right| \geq \frac{\gamma_{1}}{\gamma_{2}}\left|B_{X}(x, a)\right|\left(\frac{\rho}{a}\right)^{n} \quad \text { for } \rho \geq a .
$$

As a consequence, if $\beta<n$ we have

$$
\begin{aligned}
& \int_{a}^{b} \frac{\rho^{\beta-1}}{\left|B_{X}(x, \rho)\right|} \mathrm{d} \rho \leq \frac{\gamma_{2}}{\gamma_{1}} \frac{a^{n}}{\left|B_{X}(x, a)\right|} \int_{a}^{b} \frac{\rho^{\beta-1}}{\rho^{n}} \mathrm{~d} \rho=\frac{\gamma_{2}}{\gamma_{1}} \frac{a^{n}}{\left|B_{X}(x, a)\right|}\left(\frac{a^{\beta-n}-b^{\beta-n}}{n-\beta}\right) \\
& \quad \leq \frac{\gamma_{2}}{(n-\beta) \gamma_{1}} \frac{a^{n}}{\left|B_{X}(x, a)\right|} a^{\beta-n}=C_{4}(\beta) \frac{a^{\beta}}{\left|B_{X}(x, a)\right|}, \quad \text { where } C_{4}(\beta):=\frac{\gamma_{2}}{(n-\beta) \gamma_{1}} .
\end{aligned}
$$

The case $\beta>n$ is completely analogous. Finally, if $\beta=n$, the above computation has to be modified according to $\int_{a}^{b} \frac{\rho^{\beta-1}}{\rho^{n}} \mathrm{~d} \rho=\log \left(\frac{b}{a}\right)$. This ends the proof.

In due course of the arguments of this section, we have incidentally proved the following:

Corollary 4.11. Let $n>2$. For every integer $r \geq 0$, there exists $C_{3}=C_{3}(r)>0$ such that

$$
\int_{\mathbb{R}^{p}} d_{\widetilde{X}}^{2-Q-r}\left((x, 0)^{-1} *(y, \eta)\right) \mathrm{d} \eta \leq C_{3} \frac{d_{X}(x, y)^{2-r}}{\left|B_{X}\left(x, d_{X}(x, y)\right)\right|},
$$

for every $x, y \in \mathbb{R}^{n}$ with $x \neq y$. 


\section{Global lower estimates of $\Gamma$}

The aim of this section is to prove the following

Theorem 5.1. Let $\Gamma$ be the fundamental solution of $\mathcal{L}$ as in (1.5); let us suppose that $n>2$. Then, there exists a (structural) constant $C>0$ such that

$$
\Gamma(x ; y) \geq C \frac{d_{X}(x, y)^{2}}{\left|B_{X}\left(x, d_{X}(x, y)\right)\right|} \quad \text { for every } x \neq y \text { in } \mathbb{R}^{n} .
$$

In particular, for every fixed $x \in \mathbb{R}^{n}$ we have

$$
\lim _{y \rightarrow x} \Gamma(x ; y)=\infty .
$$

We observe that the function in the right-hand side of (5.1) is not symmetric, as is instead true of $\Gamma(x ; y)$; on the other hand, an inequality analogous to (5.1) holds true with interchanged $x$ and $y$, as we already pointed out in Remark 4.2 .

The proof of Theorem 5.1 relies on the next two propositions.

Proposition 5.2. Let $K \subset \mathbb{R}^{n}$ be any compact neighborhood of the origin. Then there exist a positive number $R=R(K)$ and a (structural) constant $C_{1}>0$ such that

$$
\Gamma(x ; y) \geq C_{1} \int_{d_{X}(x, y)}^{R} \frac{\rho}{\left|B_{X}(x, \rho)\right|} \mathrm{d} \rho,
$$

for every $x, y \in K$ with $x \neq y$. More precisely, $R$ can be chosen in such a way that $d_{X}(x, y)<R / 2$ for all $x, y \in K$.

Proposition 5.3. There exists a (structural) constant $C_{2}>0$ such that, for all $x \in \mathbb{R}^{n}$, one has

$$
\int_{a}^{b} \frac{\rho}{\left|B_{X}(x, \rho)\right|} \mathrm{d} \rho \geq C_{2} \frac{a^{2}}{\left|B_{X}(x, a)\right|}, \quad \text { for any } 0<a<b / 2 .
$$

Before giving the proofs of Propositions 5.2 and 5.3. we show how they together provide the

Proof of Theorem 5.1. The proof of (5.1) is a local-to-global argument, via homogeneity. Gathering together (5.3) and (5.4) (this is legitimate, since $d_{X}(x, y)<R / 2$ for all $x, y \in K$ ), one gets

$$
\Gamma(x ; y) \geq C \frac{d_{X}(x, y)^{2}}{\left|B_{X}\left(x, d_{X}(x, y)\right)\right|} \text { for every } x \neq y \text { in } K,
$$

where $C=C_{1} C_{2}$ and $K$ is some fixed compact neighborhood of the origin in $\mathbb{R}^{n}$.

Next we apply Remark 3.1 with the choices of $m, \Omega, M_{\lambda}$ as in the proof of Theorem 4.1, and with the functions $G, F$ given by the two members of the inequality (5.5) (valid on $(K \times K) \cap \Omega$ ), as these functions are both $M_{\lambda}$-homogeneous of degree $2-q$ (a consequence of (1.8) and (2.2)).

Finally, we prove the blow-up property in (5.2). Owing to Theorem B, we have

$$
\liminf _{y \rightarrow x} \Gamma(x ; y) \stackrel{\text { (5.1) }}{\geq} C \liminf _{y \rightarrow x} \frac{d_{X}(x, y)^{2}}{\left|B_{X}\left(x, d_{X}(x, y)\right)\right|} \stackrel{\text { 2.7) }}{\geq} \frac{C}{\gamma_{2}} \liminf _{y \rightarrow x} \frac{d_{X}(x, y)^{2}}{\sum_{k=n}^{q} f_{k}(x) d_{X}(x, y)^{k}}
$$

The latter $\lim \inf$ is $\infty$, due to the assumption $n>2$ and the fact that $f_{k} \geq 0$ (and $f_{q}>0$ ).

We then give the proofs of Propositions 5.2 and 5.3 .

Proof of Proposition 5.2. In this proof we make use of (2.9) in Theorem $\mathrm{C}$ (with the choice $y=x$ ): there exists a structural constant $c_{2}>0$ such that

$$
\left|\left\{\eta \in \mathbb{R}^{p}: d_{\widetilde{X}}((y, \eta),(y, \xi))<r\right\}\right| \geq c_{2} \frac{\left|B_{\widetilde{X}}((y, \xi), r)\right|}{\left|B_{X}(y, r)\right|}, \quad \forall y \in \mathbb{R}^{n}, \quad \xi \in \mathbb{R}^{p}, r>0 .
$$

Let $K$ be any fixed compact neighborhood of $0 \in \mathbb{R}^{n}$, and let us choose $R=R(K)>0$ such that

$$
d_{X}(x, y)<\frac{R}{2} \quad \text { for any } x, y \in K \text {. }
$$


Next, we fix different points $x, y$ in $K$ and we let $k \geq 1$ be the unique integer such that

$$
\frac{R}{2^{k+1}} \leq d_{X}(x, y)<\frac{R}{2^{k}} .
$$

The latter and (2.4) ensure that

$$
\frac{R}{2^{k+1}} \leq d_{\widetilde{X}}\left((x, 0)^{-1} *(y, \eta)\right), \quad \text { for every } \eta \in \mathbb{R}^{p} .
$$

By the representation (1.7) of $\Gamma$ and owing to (4.22), we have

$$
\begin{aligned}
\Gamma(x ; y) & \geq c^{-1} \int_{\mathbb{R}^{p}} d_{\widetilde{X}}^{2-Q}\left((x, 0)^{-1} *(y, \eta)\right) \mathrm{d} \eta \\
& \geq c^{-1} \int_{\left\{\eta: d_{\widetilde{X}}((x, 0),(y, \eta))<2 R\right\}} d_{\widetilde{X}}^{2-Q}\left((x, 0)^{-1} *(y, \eta)\right) \mathrm{d} \eta \\
& =c^{-1} \sum_{j=0}^{k+1} \int_{\Omega_{j}} d_{\widetilde{X}}^{2-Q}\left((x, 0)^{-1} *(y, \eta)\right) \mathrm{d} \eta=:(\star),
\end{aligned}
$$

where we have used (5.8), together with the notation

$$
\Omega_{j}:=\left\{\eta \in \mathbb{R}^{p}: \frac{R}{2^{j}} \leq d_{\widetilde{X}}((x, 0),(y, \eta))<\frac{R}{2^{j-1}}\right\} .
$$

From the very definition of $\Omega_{j}$ we then have

$$
(\star) \geq c^{-1} \sum_{j=0}^{k+1}\left(\frac{R}{2^{j-1}}\right)^{2-Q}\left|\Omega_{j}\right| \geq c^{-1} \sum_{j=0}^{k}\left(\frac{R}{2^{j-1}}\right)^{2-Q}\left|\Omega_{j}\right|=:(2 \star) .
$$

We now claim the following assertion: for any $j \in\{0, \ldots, k\}$, there exists $\eta_{j} \in \mathbb{R}^{p}$ such that

$$
\left\{\eta \in \mathbb{R}^{p}: d_{\widetilde{X}}\left((y, \eta),\left(y, \eta_{j}\right)\right)<\frac{R}{2^{j+1}}\right\} \subseteq \Omega_{j} .
$$

Indeed, we first observe that (see (2.4)) the projection of $B_{\widetilde{X}}\left((x, 0), R / 2^{k}\right)$ ) onto $\mathbb{R}^{n}$ is precisely $B_{X}\left(x, R / 2^{k}\right)$; thus, being $y \in B_{X}\left(x, R / 2^{k}\right)$ (see (5.7)), there exists $\bar{\eta} \in \mathbb{R}^{p} \backslash\{0\}$ such that

$$
d_{\widetilde{X}}((x, 0),(y, \bar{\eta}))<R / 2^{k} .
$$

We consider the function $g:[1, \infty) \rightarrow(0, \infty)$ defined as follows

$$
g(t):=d_{\widetilde{X}}((x, 0),(y, t \bar{\eta})) .
$$

Fixing $j \in\{0, \ldots, k\}$, from (5.10) we get $g(1)<R / 2^{k} \leq R / 2^{j}$; on the other hand, for $t$ large enough, the point $(y, t \bar{\eta})$ cannot lie in $\bar{B}_{\tilde{X}}\left((x, 0), R / 2^{j-1}\right)$, i.e., $g(t)>R / 2^{j-1}$. As a consequence (by continuity), there exists $t_{j} \geq 1$ such that

$$
g\left(t_{j}\right)=\frac{1}{2}\left(\frac{R}{2^{j}}+\frac{R}{2^{j-1}}\right)=\frac{3}{2} \frac{R}{2^{j}} .
$$

Setting $\eta_{j}:=t_{j} \bar{\eta}$, the above equality means that

$$
d_{\tilde{X}}\left((x, 0),\left(y, \eta_{j}\right)\right)=\frac{3}{2} \frac{R}{2^{j}} .
$$

As simple argument based on the triangle inequality proves that

$$
B_{\widetilde{X}}\left(\left(y, \eta_{j}\right), R / 2^{j+1}\right) \subseteq B_{\widetilde{X}}\left((x, 0), R / 2^{j-1}\right) \backslash B_{\widetilde{X}}\left((x, 0), R / 2^{j}\right),
$$

which readily implies (5.9). Owing to (5.6), the latter gives

$$
\left|\Omega_{j}\right| \geq\left|\left\{\eta \in \mathbb{R}^{p}: d_{\widetilde{X}}\left((y, \eta),\left(y, \eta_{j}\right)\right)<\frac{R}{2^{j+1}}\right\}\right| \geq c_{2} \frac{\left|B_{\widetilde{X}}\left(\left(y, \eta_{j}\right), R / 2^{j+1}\right)\right|}{\left|B_{X}\left(y, R / 2^{j+1}\right)\right|} .
$$


If we insert this in $(2 \star)$, we get

$$
\begin{aligned}
(2 \star) & \geq \frac{c_{2}}{c} \sum_{j=0}^{k}\left(\frac{R}{2^{j-1}}\right)^{2-Q} \cdot \frac{\left|B_{\widetilde{X}}\left(\left(y, \eta_{j}\right), R / 2^{j+1}\right)\right|}{\left|B_{X}\left(y, R / 2^{j+1}\right)\right|} \\
& \stackrel{\text { (2.5) }}{=} \frac{c_{2} \omega_{Q}}{c 4^{Q}} \sum_{j=0}^{k} \frac{\left(R / 2^{j-1}\right)^{2}}{\left|B_{X}\left(y, R / 2^{j+1}\right)\right|} \geq C_{1}^{\prime} \sum_{j=0}^{k} \frac{\left(R / 2^{j}\right)^{2}}{\left|B_{X}\left(y, R / 2^{j}\right)\right|}=:(3 \star),
\end{aligned}
$$

where $C_{1}^{\prime}=\frac{4 c_{2} \omega_{Q}}{c 4^{Q}}$. We now argue as in the proof of Lemma 4.9, thus getting

$$
\begin{aligned}
& (3 \star) \geq \frac{\gamma_{1} C_{1}^{\prime}}{\gamma_{2}} \int_{R / 2^{k}}^{2 R} \frac{\rho}{\left|B_{X}(y, \rho)\right|} \mathrm{d} \rho \stackrel{(5.7)}{\geq} \frac{\gamma_{1} C_{1}^{\prime}}{\gamma_{2}} \int_{2 d_{X}(x, y)}^{2 R} \frac{\rho}{\left|B_{X}(y, \rho)\right|} \mathrm{d} \rho \\
& =\frac{4 \gamma_{1} C_{1}^{\prime}}{\gamma_{2}} \int_{d_{X}(x, y)}^{R} \frac{t}{\left|B_{X}(y, 2 t)\right|} \mathrm{d} t \stackrel{\sqrt{2.30}}{\geq} \frac{4 \gamma_{1} C_{1}^{\prime}}{\gamma_{2} C_{d}} \int_{d_{X}(x, y)}^{R} \frac{t}{\left|B_{X}(y, t)\right|} \mathrm{d} t .
\end{aligned}
$$

Summing up, we have proved that (setting $C_{1}=\frac{4 \gamma_{1} C_{1}^{\prime}}{\gamma_{2} C_{d}}$ )

$$
\Gamma(x ; y) \geq C_{1} \int_{d_{X}(x, y)}^{R} \frac{t}{\left|B_{X}(y, t)\right|} \mathrm{d} t, \quad \text { for every } x \neq y \text { in } K
$$

finally, by interchanging $x$ and $y$ and bearing in mind that $\Gamma$ is symmetric, we get (5.3).

We are left with the

Proof of Proposition 5.3. We fix any $x \in \mathbb{R}^{n}$ and we consider the integrand function in (5.4):

$$
(0, \infty) \ni \rho \mapsto \frac{\rho}{\left|B_{X}(x, \rho)\right|}
$$

In the proof of Lemma 4.9 we showed that, for every $\rho>0$, one has

$$
\frac{1}{\gamma_{2}} \phi(\rho) \leq \frac{\rho}{\left|B_{X}(x, \rho)\right|} \leq \frac{1}{\gamma_{1}} \phi(\rho), \quad \text { where } \phi(\rho):=\left(\sum_{h=n}^{q} f_{h}(x) \rho^{h-1}\right)^{-1}
$$

Moreover, we observe that $\phi$ enjoys the following properties:

(1) it is non-negative and monotone decreasing;

(2) it is reverse doubling, i.e., for every $r>0$ one has

$$
\phi(2 r) \geq \alpha \phi(r) \quad \text { with } \alpha:=2^{1-q} .
$$

In order to get (5.4), it is sufficient to prove this claim: any function $\phi$ with the above properties (1) and (2) also satisfies the following estimate

$$
\int_{a}^{b} \phi(\rho) \mathrm{d} \rho \geq \alpha^{2} a \phi(a), \quad \text { for any } 0<a<b / 2 .
$$

This claim will prove (5.4) as follows:

$$
\begin{aligned}
& \int_{a}^{b} \frac{\rho}{\left|B_{X}(x, \rho)\right|} \mathrm{d} \rho \stackrel{\sqrt{5.11}}{\geq} \frac{1}{\gamma_{2}} \int_{a}^{b} \phi(\rho) \mathrm{d} \rho \stackrel{\sqrt{5.12}}{\geq} \frac{\alpha^{2}}{\gamma_{2}} a \phi(a) \\
& \stackrel{\text { (5.11) }}{\geq} C_{2} \frac{a^{2}}{\left|B_{X}(x, a)\right|} \quad \text { with } C_{2}=\frac{\alpha^{2} \gamma_{1}}{\gamma_{2}} \text {. }
\end{aligned}
$$

We are left to prove the above claim. Let $a \in(0, b / 2)$ be fixed, and let $k \in \mathbb{N}$ be such that

$$
\frac{b}{2^{k+1}}<a \leq \frac{b}{2^{k}} .
$$


This gives the following computation

$$
\begin{aligned}
\int_{a}^{b} \phi(\rho) \mathrm{d} \rho & \geq \int_{b / 2^{k}}^{b} \phi(\rho) \mathrm{d} \rho=\sum_{j=1}^{k} \int_{b / 2^{j}}^{b / 2^{j-1}} \phi(\rho) \mathrm{d} \rho \geq \sum_{j=1}^{k} \phi\left(b / 2^{j-1}\right) \frac{b}{2^{j}} \\
& \geq \phi\left(b / 2^{k-1}\right) \frac{b}{2^{k}} \geq \alpha^{2} \phi\left(b / 2^{k+1}\right) \frac{b}{2^{k}} \geq \alpha^{2} a \phi(a) .
\end{aligned}
$$

Here, we repeatedly used (1) and (2), and (5.13).

Example 5.4. Let us consider the two vector fields on $\mathbb{R}^{n}(n \geq 3)$

$$
X_{1}=\partial_{x_{1}}, \quad X_{2}=x_{1} \partial_{x_{2}}+x_{2} \partial_{x_{3}}+\ldots+x_{n-1} \partial_{x_{n}}
$$

and the corresponding PDO $\mathcal{L}=X_{1}^{2}+X_{2}^{2}$ on $\mathbb{R}^{n}$. The vector fields $X_{1}$ and $X_{2}$ are homogeneous of degree 1 with respect to the dilations

$$
\delta_{\lambda}(x)=\left(\lambda x_{1}, \lambda^{2} x_{2}, \lambda^{3} x_{3}, \ldots, \lambda^{n} x_{n}\right),
$$

which gives $q=n(n+1) / 2 \geq 6$. Observe that

$$
Y_{k}:=\left[\left[[X_{1}, \underbrace{\left.\left.X_{2}\right], X_{2}\right] \cdots X_{2}}_{k \text { times }}]=\partial_{x_{k+1}}, \quad \text { for } k=1, \ldots, n-1 .\right.\right.
$$

Thus, assumptions (H1)-to-(H3) of Section 1 are satisfied (here $N=n+1)$. Following the construction in Remark 2.1, the possible choices of a basis of $\mathbb{R}^{n}$ are:

- $X_{1}, Y_{1}, \ldots, Y_{n-1}$, which has weight $1+2+\cdots+n=n(n+1) / 2$ and $f_{q}=1$;

- $X_{1}, X_{2}$ and $n-2$ out of the $n-1$ commutators $Y_{j}$. Denoting by $\mathcal{B}_{j}(j=1, \ldots, n-1)$ the choice containing all the $Y_{k}$ 's except for $k=j$, we have

$$
\left|\mathcal{B}_{j}\right|=1+1+(2+3+\cdots+n)-(j+1)=q-j, \quad \text { and } \quad f_{q-j}(x)=\left|x_{j}\right| .
$$

Hence

$$
\Lambda(x, \rho)=\rho^{q}+\sum_{j=1}^{n-1}\left|x_{j}\right| \rho^{q-j} .
$$

Thus, owing to our Theorem 1.3.(III), we have the global estimates

$$
\Gamma(x ; y) \approx\left(d_{X}(x, y)^{q-2}+\sum_{j=1}^{n-1}\left|x_{j}\right| d_{X}(x, y)^{q-j-2}\right)^{-1} .
$$

6. THe CASE $n=2$

In this section we investigate the case $n=2$, not covered in the previous sections. The obstructions imposed by taking $n=2$ are not only technical: jointly with the trivial example of the logarithmic fundamental solution of Laplace's operator in $\mathbb{R}^{2}$, we also have less trivial examples of fundamental solutions which (near the diagonal, at least) exhibit either a "logarithmic-type" behavior or a "power-like" behavior, depending on the point. As we shall detail in Example 6.6, this happens for instance for the class of operators

$$
\mathcal{G}=\left(\partial_{x_{1}}\right)^{2}+\left(x_{1}^{k} \partial_{x_{2}}\right)^{2} \quad(k \in \mathbb{N})
$$

What is really pathological is the case $(n, r)=(2,0)$ in Theorem 4.1 which means to obtain a global pointwise upper estimate of $\Gamma$; likewise, Theorem 5.1 must be adapted in the case $n=2$. On the contrary, the case $n=2$ and $r \geq 1$ goes like in Theorem 4.1, as the following result shows.

Theorem 6.1. Let $n=2$ and let $\Gamma$ be the fundamental solution of $\mathcal{L}$ in (1.5). Then, for any integer $r \geq 1$, there exists $C>0$ (depending on $r$ and on the set $X=\left\{X_{1}, \ldots, X_{m}\right\}$ ) such that

$$
\left|Z_{1} \cdots Z_{r} \Gamma(x ; y)\right| \leq C \frac{d_{X}(x, y)^{2-r}}{\left|B_{X}\left(x, d_{X}(x, y)\right)\right|},
$$

for any $x, y \in \mathbb{R}^{2}$ (with $\left.x \neq y\right)$ and any choice of

$$
Z_{1}, \ldots, Z_{r} \in\left\{X_{1}^{x}, \ldots, X_{m}^{x}, X_{1}^{y}, \ldots, X_{m}^{y}\right\} .
$$


In particular, for every fixed $x \in \mathbb{R}^{2}$ we have

$$
\lim _{|y| \rightarrow \infty} Z_{1} \cdots Z_{r} \Gamma(x ; y)=0
$$

Proof. An inspection of the proofs of the results in Section 4 shows that, when $n=2$ and $r \geq 1$,

- Lemma 4.3 remains unvaried, and Proposition 4.6 follows from Lemma 4.3.

- Proposition 4.7holds unvaried: indeed, in (4.29) we have $M(K, r)<\infty$, as $n+r-2=r \geq 1$;

- Lemma 4.9 holds true for any $\beta \leq n+1=3$. This enables us to obtain Proposition 4.8 (for $n=2$ and $r \geq 1$ ), by combining Lemma 4.9 with Lemma 4.10 (with the choice $\beta=2-r<2=n)$.

Finally, with Propositions 4.6 to 4.8 at hand, the proofs of (6.1) and (6.2) follow as in Section 4 .

In the above arguments, we have incidentally proved the following:

Corollary 6.2. Let $n=2$. For every integer $r \geq 1$, there exists $C=C(r)>0$ such that

$$
\int_{\mathbb{R}^{p}} d_{\widetilde{X}}^{2-Q-r}\left((x, 0)^{-1} *(y, \eta)\right) \mathrm{d} \eta \leq C \frac{d_{X}(x, y)^{2-r}}{\left|B_{X}\left(x, d_{X}(x, y)\right)\right|},
$$

for every $x, y \in \mathbb{R}^{2}$ with $x \neq y$.

As for the estimates of $\Gamma$, both from above and from below, one cannot expect global results: this is due to the fact that, when $n=2, \Gamma$ can behave logarithmically near the diagonal and much differently off the diagonal. Thus, we are firstly content with the following estimates (Theorem 6.3) valid on compact sets $K$, uniformly as $x$ and $y$ vary in $K$. Secondly, we shall prove optimal estimates (in that both upper and lower bounds are of the same form) near the diagonal and depending upon the pole $x$ (Theorem 6.5).

Theorem 6.3. Let the assumptions of Theorem 6.1 apply, and let $K \subseteq \mathbb{R}^{n}$ be any compact set. Then, there exist a structural constant $C_{0}>0$ and a real $R_{0}=R_{0}(K)>0$ such that

$$
\Gamma(x ; y) \leq C_{0} \frac{d_{X}(x, y)^{2}}{\left|B_{X}\left(x, d_{X}(x, y)\right)\right|} \cdot \log \left(\frac{R_{0}}{d_{X}(x, y)}\right),
$$

for every $x, y \in K$ with $x \neq y$.

Moreover, there exist positive numbers $C_{1}=C_{1}(K)$ and $R_{1}=R_{1}(K)$ such that

$$
\Gamma(x ; y) \geq C_{1} \log \left(\frac{R_{1}}{d_{X}(x, y)}\right)
$$

for every $x, y \in K$ with $x \neq y$. In particular, for every fixed $x \in \mathbb{R}^{2}$ we have

$$
\lim _{y \rightarrow x} \Gamma(x ; y)=\infty .
$$

It can be noticed that a serious obstruction in globalizing (6.4)- 6.5 is the lack of homogeneity of logarithmic members in the right-hand sides. However, this does not prevent $\Gamma(x ; y)$ from vanishing as $|y|$ (or $|x|)$ goes to infinity, as is proved in [10] (see (3) in Theorem A).

Proof. We begin by establishing (6.4). Scrutinizing the proofs of the results in Section 4 with $(n, r)=(2,0)$ we see that

- Proposition 4.6 follows from the representation formula (1.7);

- Proposition 4.7 holds unvaried, as $n+r-2=0$ (see again (4.29));

- Lemma 4.9 holds true with $\beta=2<n+1$. 
Then, fixing any compact set $K$, we obtain the following chain of inequalities:

$$
\begin{aligned}
& \Gamma(x ; y) \stackrel{\text { 4.21] }}{\leq} c \int_{\mathbb{R}^{p}} d_{\tilde{X}}^{2-Q}\left((x, 0)^{-1} *(y, \eta)\right) \mathrm{d} \eta \\
& \quad=c \int_{|\eta| \geq 1} d_{\tilde{X}}^{2-Q}\left((x, 0)^{-1} *(y, \eta)\right) \mathrm{d} \eta+c \int_{|\eta|<1} d_{\widetilde{X}}^{2-Q}\left((x, 0)^{-1} *(y, \eta)\right) \mathrm{d} \eta \\
& \stackrel{4.25 \mid}{\leq} c_{1} \frac{d_{X}(x, y)^{2}}{\left|B_{X}\left(x, d_{X}(x, y)\right)\right|}+c \int_{|\eta|<1} d_{\tilde{X}}^{2-Q}\left((x, 0)^{-1} *(y, \eta)\right) \mathrm{d} \eta \\
& \stackrel{4.30)}{\leq} c_{1} \frac{d_{X}(x, y)^{2}}{\left|B_{X}\left(x, d_{X}(x, y)\right)\right|}+c_{2} \int_{d_{X}(x, y)}^{R_{0}} \frac{\rho}{\left|B_{X}(x, \rho)\right|} \mathrm{d} \rho .
\end{aligned}
$$

From this, we obtain the upper estimate in (6.4) by applying Lemma 4.10 in the case $\beta=n=2$, and by possibly enlarging $R_{0}$ in such a way that $R_{0}>3 \sup \left\{d_{X}(x, y): x, y \in K\right\}$.

We then prove (6.5). To this end, let $R=R(K)>0$ be as in Proposition 5.2, If $x, y \in K$ and $d_{X}(x, y) \geq 1$ (whence $R \geq 2$ ), then (6.5) is trivially satisfied with the choice

$$
C_{1}(K):=\frac{1}{\log (R)} \inf \left\{\Gamma(x ; y): x, y \in K \text { and } d_{X}(x, y) \geq 1\right\}>0 .
$$

If, instead, $x, y \in K$ and $0<d_{X}(x, y)<1$, an inspection of the proof of Proposition 5.2 shows that (5.3) is valid also in the case $n=2$, thus giving

$$
\Gamma(x ; y) \geq C \int_{d_{X}(x, y)}^{R} \frac{\rho}{\left|B_{X}(x, \rho)\right|} \mathrm{d} \rho \geq C \int_{d_{X}(x, y)}^{\min \{R, 1\}} \frac{\rho}{\left|B_{X}(x, \rho)\right|} \mathrm{d} \rho=(\star) .
$$

On the other hand, if $0<\rho \leq 1$, by Theorem B we have

$$
|B(x, \rho)| \leq \gamma_{2} \sum_{h=2}^{q} f_{h}(x) \rho^{h} \leq \gamma_{2}^{\prime} \rho^{2}, \quad \text { where } \gamma_{2}^{\prime}:=\gamma_{2}\left(f_{q}+\max _{x \in K} \sum_{h=2}^{q-1} f_{h}(x)\right) .
$$

Notice that $\gamma_{2}^{\prime} \geq f_{q}>0$. Thus, we obtain

$$
(\star) \geq \frac{C}{\gamma_{2}^{\prime}} \int_{d_{X}(x, y)}^{\min \{R, 1\}} \frac{1}{\rho} \mathrm{d} \rho=\frac{C}{\gamma_{2}^{\prime}} \log \left(\frac{\min \{R, 1\}}{d_{X}(x, y)}\right) .
$$

Summing up, (6.5) is satisfied by possibly replacing $C_{1}$ with $\min \left\{C_{1}, C / \gamma_{2}^{\prime}\right\}$, and with the choice $R_{1}:=\min \{R(K), 1\}$. Finally, we show (6.6). We apply (6.5) with the choice $K=\{y:\|y-x\| \leq 1\}$ :

$$
\liminf _{y \rightarrow x} \Gamma(x ; y) \geq C_{1}(K) \liminf _{y \rightarrow x} \log \left(\frac{R_{1}(K)}{d_{X}(x, y)}\right),
$$

for a suitable $R_{1}(K)>0$. The latter liminf is clearly $\infty$, and the proof is complete.

Remark 6.4. The main feature of Theorem 6.3 is to provide uniform estimates, valid as both $x$ and $y$ may vary (in some compact set). In a different spirit, we next consider the case when $x$ is fixed and $y$ is near the pole $x$. We shall see in Theorem 6.5 that a different (and more precise) behavior arises. Roughly put, the more "rigid" situation of a fixed pole $x$ will allow us to obtain upper and lower estimates with the same type of bounding functions (compare (6.7) to (6.4)-(6.5)). However, as we shall show in Example 6.7. this is not in contrast with the different nature of the uniform estimates of $\Gamma$, where the variability of the lower and upper bounds (6.4)-(6.5) depicts the more general situation of both variable $x$ 's and $y$ 's.

Theorem 6.5. Let $n=2$ and let the assumptions of Theorem 6.1 apply. Let $f_{2}$ be the nonnegative function introduced in Theorem B. Then, for any $x \in \mathbb{R}^{n}$, there exist positive constants $\gamma_{1}(x), \gamma_{2}(x)$ and a small $\varepsilon(x)<1$ (all depending on $x$ only) such that

$$
\gamma_{1}(x) F(x, y) \leq \Gamma(x ; y) \leq \gamma_{2}(x) F(x, y),
$$


for any $y$ such that $0<d_{X}(x, y)<\varepsilon(x)$, where

$$
F(x, y)= \begin{cases}\log \left(\frac{1}{d_{X}(x, y)}\right) & \text { if } f_{2}(x)>0 \\ \frac{d_{X}(x, y)^{2}}{\mid B_{X}\left(x, d_{X}(x, y)\right)} & \text { if } f_{2}(x)=0\end{cases}
$$

In the case $f_{2}(x)=0$, the estimate (6.7) holds true with $\varepsilon(x)=1 / 2$ and $\gamma_{1}(x)$ independent of $x$. In the case $f_{2}(x)=0, F(x, y)$ diverges like $d_{X}(x, y)^{2-k}$, for some $k \in\{3, \ldots, q\}$.

Proof. Let $x \in \mathbb{R}^{n}$ be arbitrarily fixed. We take any $y \in \mathbb{R}^{n}$ conveniently close to $x$, namely

$$
0<d_{X}(x, y) \leq 1 / 2 \text {. }
$$

Next, we take a compact set $K$ which is a neighborhood of $0 \in \mathbb{R}^{n}$, and such that $K$ contains the closure of $B_{X}(x, 1 / 2)$. Finally, we choose $R_{0}(x) \gg 1$ such that

$$
d_{\tilde{X}}\left((x, 0)^{-1} *(y, \eta)\right)<\frac{R_{0}(x)}{2},
$$

uniformly for any $y$ as in (6.9) and any $|\eta| \leq 1$. This implicitly implies that $d_{X}(x, y) \leq R_{0}(x) / 2$. With all these choices, in due course of the proof of Theorem [6.3, we proved the existence of two constants $c_{1}(x), c_{2}(x)>0$ and of a structural constant $c>0$ such that

$$
c \int_{d_{X}(x, y)}^{1} \frac{\rho}{\left|B_{X}(x, \rho)\right|} \mathrm{d} \rho \leq \Gamma(x ; y) \leq c_{1}(x) \frac{d_{X}(x, y)^{2}}{\left|B_{X}\left(x, d_{X}(x, y)\right)\right|}+c_{2}(x) \int_{d_{X}(x, y)}^{R_{0}(x)} \frac{\rho}{\left|B_{X}(x, \rho)\right|} \mathrm{d} \rho .
$$

We observe that $c_{1}(x), c_{2}(x)$ depend on $x$ only through the compact set $K$ containing $x$.

On account of (2.7), we remind that (as $n=2$ ) we have nonnegative functions $f_{k}$ such that

$$
\gamma_{1} \sum_{k=2}^{q} f_{k}(x) \rho^{k} \leq\left|B_{X}(x, \rho)\right| \leq \gamma_{2} \sum_{k=2}^{q} f_{k}(x) \rho^{k}, \quad \forall x \in \mathbb{R}^{n}, \rho>0 .
$$

Then we distinguish two cases:

(I). Suppose that $f_{2}(x)>0$. Then (6.11) gives

$$
\gamma_{1} f_{2}(x) \rho^{2} \leq\left|B_{X}(x, \rho)\right| \leq \gamma_{2} \rho^{2}\left(f_{2}(x)+\sum_{k=3}^{q} f_{k}(x) \rho^{k-2}\right) .
$$

Hence the choices $\gamma_{1}(x):=\gamma_{1} f_{2}(x)$ and $\gamma_{2}(x):=\gamma_{2} \sum_{k=2}^{q} f_{k}(x) R_{0}(x)^{k-2}$ are two positive constants (only depending on $x$ ) such that

$$
\gamma_{1}(x) \rho^{2} \leq\left|B_{X}(x, \rho)\right| \leq \gamma_{2}(x) \rho^{2}, \quad \text { for any } 0<\rho \leq R_{0}(x) .
$$

Therefore, the latter inequalities give (taking $\rho=d_{X}(x, y) \leq 1 / 2<1 \ll R_{0}(x)$ )

$$
\frac{1}{\gamma_{2}(x)} \leq \frac{d_{X}(x, y)^{2}}{\left|B_{X}\left(x, d_{X}(x, y)\right)\right|} \leq \frac{1}{\gamma_{1}(x)}, \quad \forall y \in \overline{B_{X}(x, 1 / 2)} .
$$

Again by (6.12) one also has

$$
\frac{1}{\gamma_{2}(x) \rho} \leq \frac{\rho}{\left|B_{X}(x, \rho)\right|} \leq \frac{1}{\gamma_{1}(x) \rho}, \quad \text { for any } 0<\rho \leq R_{0}(x) .
$$

Thus, upon an integration of the latter, we can give a lower bound for the far left-hand side of (6.10) and an upper bound for the second summand in the far right-hand side of (6.10): gathering these bounds together with (6.13), we deduce from (6.10) the following estimates:

$$
\frac{c}{\gamma_{2}(x)} \log \left(\frac{1}{d_{X}(x, y)}\right) \leq \Gamma(x ; y) \leq \frac{c_{1}(x)}{\gamma_{1}(x)}+\frac{c_{2}(x)}{\gamma_{1}(x)} \log \left(\frac{R_{0}(x)}{d_{X}(x, y)}\right)
$$


holding true for any $y$ as in (6.9). This is sufficient ${ }^{3}$ to infer the existence of positive constants $\gamma_{i}(x)$ $(i=1,2)$ and $\varepsilon(x) \ll 1$ such that (6.7) holds, with $F(x, y)$ as in the first case of (6.8).

(II). Suppose that $f_{2}(x)=0$. In this case, (6.11) gives

$$
\gamma_{1} \sum_{k=3}^{q} f_{k}(x) r^{k} \leq\left|B_{X}(x, r)\right| \leq \gamma_{2} \sum_{k=3}^{q} f_{k}(x) r^{k}, \quad \forall x \in \mathbb{R}^{n}, r>0 .
$$

We fix $x \in \mathbb{R}^{n}$ and $r>0$, and we set $\Lambda(x, r):=\sum_{k=3}^{q} f_{k}(x) r^{k}$; from (6.14) (rewritten with $\lambda r$ replacing $r$ ) we derive that, for any $\lambda \geq 1$, one has

$$
\left|B_{X}(x, \lambda r)\right| \leq \gamma_{2} \Lambda(x, \lambda r)=\gamma_{2} \sum_{k=3}^{q} f_{k}(x) r^{k} \lambda^{k} \leq \lambda^{q} \gamma_{2} \Lambda(x, r) \stackrel{\sqrt[6.14]{\leq}}{\leq} \lambda^{q} \frac{\gamma_{2}}{\gamma_{1}}\left|B_{X}(x, r)\right| .
$$

Analogously, again for any $\lambda \geq 1$,

$$
\left|B_{X}(x, \lambda r)\right| \geq \gamma_{1} \Lambda(x, \lambda r)=\gamma_{1} \sum_{k=3}^{q} f_{k}(x) r^{k} \lambda^{k} \geq \lambda^{3} \gamma_{1} \Lambda(x, r) \stackrel{\sqrt[6.14]{\geq}}{\geq} \lambda^{3} \frac{\gamma_{1}}{\gamma_{2}}\left|B_{X}(x, r)\right| .
$$

This gives at once, for any $\lambda \geq 1$,

$$
\lambda^{3} \frac{\gamma_{1}}{\gamma_{2}}\left|B_{X}(x, r)\right| \leq\left|B_{X}(x, \lambda r)\right| \leq \lambda^{q} \frac{\gamma_{2}}{\gamma_{1}}\left|B_{X}(x, r)\right|
$$

If $\rho \geq d_{X}(x, y)>0$, we are entitled to choose $\lambda=\rho / d_{X}(x, y) \geq 1$ and $r=d_{X}(x, y)$ in (6.15), getting

$$
\frac{\gamma_{1}}{\gamma_{2}} \frac{\rho^{3}}{d_{X}(x, y)^{3}}\left|B_{X}\left(x, d_{X}(x, y)\right)\right| \leq\left|B_{X}(x, \rho)\right| \leq \frac{\gamma_{2}}{\gamma_{1}} \frac{\rho^{q}}{d_{X}(x, y)^{q}}\left|B_{X}\left(x, d_{X}(x, y)\right)\right| .
$$

The first inequality in (6.16) allows us to estimate the second summand in the far right-hand side of (6.10) in the following way:

$$
\begin{aligned}
& \int_{d_{X}(x, y)}^{R_{0}(x)} \frac{\rho}{\left|B_{X}(x, \rho)\right|} \mathrm{d} \rho \leq \frac{\gamma_{2}}{\gamma_{1}} \frac{d_{X}(x, y)^{3}}{\left|B_{X}\left(x, d_{X}(x, y)\right)\right|} \int_{d_{X}(x, y)}^{R_{0}(x)} \frac{\rho}{\rho^{3}} \mathrm{~d} \rho \\
& \quad=\frac{\gamma_{2}}{\gamma_{1}} \frac{d_{X}(x, y)^{3}}{\left|B_{X}\left(x, d_{X}(x, y)\right)\right|} \frac{R_{0}(x)-d_{X}(x, y)}{d_{X}(x, y) R_{0}(x)} \leq \frac{\gamma_{2}}{\gamma_{1}} \frac{d_{X}(x, y)^{2}}{\left|B_{X}\left(x, d_{X}(x, y)\right)\right|} .
\end{aligned}
$$

The second inequality in (6.16) gives the following estimate for the first summand in the far left-hand side of (6.10) (we are also using (6.9), so that $1 \geq 2 d_{X}(x, y)$ ):

$$
\begin{aligned}
& \int_{d_{X}(x, y)}^{1} \frac{\rho}{\left|B_{X}(x, \rho)\right|} \mathrm{d} \rho \geq \frac{\gamma_{1}}{\gamma_{2}} \frac{d_{X}(x, y)^{q}}{\left|B_{X}\left(x, d_{X}(x, y)\right)\right|} \int_{d_{X}(x, y)}^{1} \frac{\rho}{\rho^{q}} \mathrm{~d} \rho \\
& \quad \geq \frac{\gamma_{1}}{\gamma_{2}} \frac{d_{X}(x, y)^{q}}{\left|B_{X}\left(x, d_{X}(x, y)\right)\right|} \int_{d_{X}(x, y)}^{2 d_{X}(x, y)} \frac{1}{\rho^{q-1}} \mathrm{~d} \rho=\frac{\gamma_{1}\left(1-2^{2-q}\right)}{\gamma_{2}(q-2)} \frac{d_{X}(x, y)^{2}}{\left|B_{X}\left(x, d_{X}(x, y)\right)\right|} .
\end{aligned}
$$

Summing up, (6.10) gives

$$
\frac{c \gamma_{1}\left(1-2^{2-q}\right)}{\gamma_{2}(q-2)} \frac{d_{X}(x, y)^{2}}{\left|B_{X}\left(x, d_{X}(x, y)\right)\right|} \leq \Gamma(x ; y) \leq \frac{d_{X}(x, y)^{2}}{\left|B_{X}\left(x, d_{X}(x, y)\right)\right|}\left(c_{1}(x)+\frac{c_{2}(x) \gamma_{2}}{\gamma_{1}}\right) .
$$

This proves (6.7), with $F(x, y)$ as in the second case of (6.8). Incidentally, this also proves that (6.7) holds true with $\varepsilon(x)=1 / 2$ and $\gamma_{1}(x)$ independent of $x$. As for the last assertion of the theorem, this immediately follows from (6.8) and (6.14).

Example 6.6. Let us consider the vector fields on $\mathbb{R}^{2}$

$$
X_{1}=\partial_{x_{1}}, \quad X_{2}=x_{1}^{k} \partial_{x_{2}},
$$

for a fixed integer $k \geq 1$, and the corresponding PDO on $\mathbb{R}^{2}$

$$
\mathcal{L}=\partial_{x_{1}}^{2}+x_{1}^{2 k} \partial_{x_{2}}^{2} .
$$

\footnotetext{
${ }^{3}$ First we choose $\varepsilon(x)>0$ such that $\frac{c_{1}(x)}{\gamma_{1}(x)} \leq \frac{c_{2}(x)}{\gamma_{1}(x)} \log \left(\frac{R_{0}(x)}{d_{X}(x, y)}\right)$ for $0<d_{X}(x, y) \leq \varepsilon(x)$; for instance the choice $\varepsilon(x):=R_{0}(x) \exp \left(-c_{1}(x) / c_{2}(x)\right)$ does the job. Then one can use the inequality $\log \left(\frac{R_{0}(x)}{d_{X}(x, y)}\right) \leq \alpha(x) \log \left(\frac{1}{d_{X}(x, y)}\right)$ valid for $d_{X}(x, y) \leq \varepsilon(x)<1$ (and any $R_{0}(x) \geq 1$ ), if one chooses $\alpha(x)=1-\log \left(R_{0}(x)\right) / \log (\varepsilon(x))$.
} 
It is readily seen that $X_{1}$ and $X_{2}$ are homogeneous of degree 1 with respect to the dilations

$$
\delta_{\lambda}\left(x_{1}, x_{2}\right)=\left(\lambda x_{1}, \lambda^{k+1} x_{2}\right)
$$

Obviously, assumptions (H1)-to-(H3) of Section 1 are satisfied (here $q=N=k+2$ are both $>2$ ). In particular (see Remark 2.1 for the following construction), the possible bases of $\mathbb{R}^{2}$ built by commutators of $X_{1}, X_{2}$, are:

$$
\begin{array}{lll}
X_{1}=\partial_{x_{1}}, X_{2}=x_{1}^{k} \partial_{x_{2}} & \text { of weight } 2 & \text { with } f_{2}(x)=\left|x_{1}\right|^{k} \\
X_{1},\left[X_{1}, X_{2}\right]=k x_{1}^{k-1} \partial_{x_{2}} & \text { of weight } 3 & \text { with } f_{3}(x)=k\left|x_{1}\right|^{k-1} \\
X_{1},\left[X_{1},\left[X_{1}, X_{2}\right]\right]=k(k-1) x_{1}^{k-2} \partial_{x_{2}} & \text { of weight } 4 & \text { with } f_{4}(x)=k(k-1)\left|x_{1}\right|^{k-2} \\
\vdots & \vdots & \vdots \\
X_{1},\left[X_{1},\left[X_{1}, \ldots\left[X_{1}, X_{2}\right]\right]\right]=k ! \partial_{x_{2}} & \text { of weight } k+2 & \text { with } f_{k+2}=k !
\end{array}
$$

Therefore, for every $x$ and $\rho$, one has

$$
\left|B_{X}(x, \rho)\right| \approx \Lambda(x, \rho)=\left|x_{1}\right|^{k} \rho^{2}+k\left|x_{1}\right|^{k-1} \rho^{3}+k(k-1)\left|x_{1}\right|^{k-2} \rho^{4}+\cdots+k ! \rho^{k+2} .
$$

Thus, according to our Theorem 6.5, we have, when $y$ is sufficiently close to $x$,

$$
\Gamma(x ; y) \approx_{x} \begin{cases}\log \left(\frac{1}{d_{X}(x, y)}\right) & \text { if } x_{1} \neq 0, \\ \frac{1}{d_{X}(x, y)^{k}} & \text { if } x_{1}=0 .\end{cases}
$$

where $\approx_{x}$ means upper/lower bounds with constant possibly depending on the fixed $x$; this exhibits a different asymptotic behavior for $y \rightarrow x$, at different points $x$.

Example 6.6 exhibits the different behavior (logarithmical vs. power-like) of $\Gamma(x ; y)$ as $y$ approaches different poles $x$. Thus, the lower estimate (6.5) cannot be improved to become analogous to the upper estimate (6.4). A question remains open whether the upper estimate (6.4) is optimal or not: in this regard, Theorem 6.5 would induce one to think that the upper bound be either logarithmic or power-like, and it does not seem to forecast the presence of a product of these bounds. However, the next Example 6.7 will show that, if $x$ and $y$ can vary the same time (which is possible, in the case of uniform estimates, as we already pointed out in Remark 6.4), then one can exactly obtain the product of a logarithm and a power of $d_{X}(x, y)$.

Indeed, when one takes $k=1$ in Example 6.6. the global fundamental solution of

$$
\mathcal{G}=\left(\partial_{x_{1}}\right)^{2}+\left(x_{1} \partial_{x_{2}}\right)^{2}
$$

can be explicitly computed, so that we can verify the optimality of our estimates in Theorems 6.3 and 6.5. in this sense, Example6.6 differs from Example 6.7 in that in the latter we start from what is known about $\Gamma$ and then we check our results, rather than confining ourselves in showing what our results state.

Example 6.7 (The Grushin case for $k=1$ ). Take $k=1$ in Example 6.6, and consider the associated vector fields $X_{1}=\partial_{x_{1}}$ and $X_{2}=x_{1} \partial_{x_{2}}$. Due to the results by Franchi, Lanconelli performed for the vector fields $\partial_{x_{1}}$ and $\left|x_{1}\right| \partial_{x_{2}}$ in [21] (see also Kogoj, Lanconelli [26]) 4 one can get the explicit estimate for the Carnot-Carathéodory distance $d_{X}$ :

$$
d_{X}(x, y) \approx\left|x_{1}-y_{1}\right|+\sqrt{\left|x_{1}\right|^{2}+\left|x_{2}-y_{2}\right|}-\left|x_{1}\right|, \quad \text { for every } x, y \in \mathbb{R}^{2} .
$$

Moreover, Theorem B gives (see also (6.17) with $k=1$ )

$$
\left|B_{X}\left(x, d_{X}(x, y)\right)\right| \approx\left|x_{1}\right| d_{X}(x, y)^{2}+d_{X}(x, y)^{3}, \quad \text { for every } x, y \in \mathbb{R}^{2} .
$$

\footnotetext{
${ }^{4}$ It is not difficult to recognize that the CC-distance induced by $\partial_{x_{1}}$ and $\left|x_{1}\right| \partial_{x_{2}}$ coincides with $d_{X}$.

${ }^{5}$ Here and in the sequel, $\approx$ means upper/lower estimates, up to some universal constants; the variant $\approx_{x}$ means that upper/lower estimates are true, modulo constants possibly depending on $x$. With the notation ' $f(x) \sim g(x)$ as $x \rightarrow x_{0}$ ' we mean, as usual, $f(x)=g(x) \omega(x)$ with $\omega(x) \rightarrow 1$ as $x \rightarrow x_{0}$.
} 
With the notation in (2.7), we have $f_{2}(x)=\left|x_{1}\right|$ and $f_{3}(x) \equiv 1$. Furthermore, the lifting Carnot group as in Theorem $\mathrm{A}$ is $\mathbb{G}=\left(\mathbb{R}^{3} \equiv \mathbb{R}_{x}^{2} \times \mathbb{R}_{\xi}, *\right)$, where

$$
\left(x_{1}, x_{2}, \xi\right) *\left(x_{1}^{\prime}, x_{2}^{\prime}, \xi^{\prime}\right)=\left(x_{1}+x_{1}^{\prime}, x_{2}+x_{2}^{\prime}+x_{1} \xi^{\prime}, \xi+\xi^{\prime}\right) .
$$

Thus, the vector fields $\widetilde{X}_{1}, \widetilde{X}_{2}$ lifting $X_{1}$ and $X_{2}$ are

$$
\tilde{X}_{1}=\partial_{x_{1}}, \quad \tilde{X}_{2}=x_{1} \partial_{x_{2}}+\partial_{\xi} .
$$

The operator $\mathcal{L}=X_{1}^{2}+X_{2}^{2}$ is lifted to the sublaplacian $\mathcal{L}_{\mathbb{G}}=\widetilde{X}_{1}^{2}+\widetilde{X}_{2}^{2}$. The latter is (up to a change of variable) the Kohn Laplacian on the first Heisenberg group $\mathbb{H}^{1}$; after simple computations (manipulating the well-known fundamental solution for the Kohn Laplacian on $\mathbb{H}^{1}$ ), one finds the fundamental solution with pole at the origin of $\mathcal{L}_{\mathbb{G}}$ :

$$
\Gamma_{\mathbb{G}}(x, \xi)=\gamma_{0}\left(\left(x_{1}^{2}+\xi^{2}\right)^{2}+16\left(x_{2}-\frac{1}{2} x_{1} \xi\right)^{2}\right)^{-1 / 2}, \quad(x, \xi) \neq(0,0),
$$

where $\gamma_{0}>0$ is a suitable constant. According to Theorem A, the function

$$
\Gamma\left(x_{1}, x_{2} ; y_{1}, y_{2}\right)=\gamma_{0} \int_{\mathbb{R}} \frac{\mathrm{d} \eta}{\sqrt{\left(\left(x_{1}-y_{1}\right)^{2}+\eta^{2}\right)^{2}+4\left(2 x_{2}-2 y_{2}+\eta\left(x_{1}+y_{1}\right)\right)^{2}}},
$$

is the unique fundamental solution for the Grushin operator $\mathcal{L}$ vanishing at infinity. The integral in (6.20) can be expressed in terms of elliptic functions in a standard way: more precisely,

$$
\Gamma(x ; y)=\frac{\gamma_{0} \sqrt{2}}{\sqrt[4]{\left(x_{1}^{2}+y_{1}^{2}\right)^{2}+4\left(x_{2}-y_{2}\right)^{2}}} \cdot \mathrm{K}\left(\frac{1}{2}+\frac{x_{1} y_{1}}{\sqrt{\left(x_{1}^{2}+y_{1}^{2}\right)^{2}+4\left(x_{2}-y_{2}\right)^{2}}}\right),
$$

where $\mathrm{K}$ denotes the complete elliptic integral of the first kind, that is,

$$
\mathrm{K}(m):=\int_{0}^{\pi / 2} \frac{\mathrm{d} \theta}{\sqrt{1-m \sin ^{2}(\theta)}}, \quad \text { for }-1<m<1 .
$$

This gives back a formula obtained by Greiner [22] (see also [6, 7, 8, 5]). In the sequel, we write (6.21) as $\Gamma(x ; y)=H(x, y) \cdot \mathrm{K}(x, y)$, with the obvious meaning. When $x_{1}=0$ (6.21) gives

$$
\Gamma\left(0, x_{2} ; y_{1}, y_{2}\right)=\frac{\gamma_{0} \sqrt{2} \mathrm{~K}(1 / 2)}{\sqrt[4]{y_{1}^{4}+4\left(x_{2}-y_{2}\right)^{2}}} .
$$

Setting $\Omega:=\left\{x \in \mathbb{R}^{2}: x_{1} \neq 0\right\}$, if $x$ is fixed in $\Omega$, then only the factor $\mathrm{K}(x, y)$ diverges as $y \rightarrow x$ (while $H(x, y)$ remains bounded); conversely, if $x$ is fixed outside $\Omega$ (i.e., $x_{1}=0$ ), only the factor $H(x, y)$ diverges as $y \rightarrow x($ while $\mathrm{K}(x, y)=\mathrm{K}(1 / 2))$.

In Remark 6.8, we will show that

$$
\mathrm{K}(m) \sim-\frac{1}{2} \ln (1-m) \quad \text { as } m \rightarrow 1^{-} .
$$

Thus, fixing $x \in \mathbb{R}^{2}$, (6.22) gives a very precise asymptotic behavior of $\Gamma(y ; x)$ as $y \rightarrow x$ :

$$
\Gamma(x ; y) \sim \begin{cases}-\frac{\gamma_{0}}{2\left|x_{1}\right|} \log \left(\frac{1}{2}-\frac{x_{1} y_{1}}{\sqrt{\left(x_{1}^{2}+y_{1}^{2}\right)^{2}+4\left(x_{2}-y_{2}\right)^{2}}}\right), & \text { if } x_{1} \neq 0 \\ \frac{\gamma_{0} \sqrt{2} \mathrm{~K}(1 / 2)}{\sqrt[4]{y_{1}^{4}+4\left(x_{2}-y_{2}\right)^{2}}}, & \text { if } x_{1}=0 .\end{cases}
$$

We now compare the above formulas with our estimates in Theorems 6.3 and 6.5 .

- Theorem 6.5: The estimates in (6.7) have full feedback from (6.23). Indeed, due to (6.19), the function $F(x, y)$ in $(\underline{6.8})$ is in the present case

$$
F(x, y)= \begin{cases}\log \left(\frac{1}{d_{X}(x, y)}\right) & \text { if } x_{1} \neq 0 \\ \frac{1}{d_{X}(x, y)} & \text { if } x_{1}=0\end{cases}
$$


Thus (6.7) is in accordance with (6.23) for the following reasons (here we used (6.18) and some Taylor approximation (6):

$$
\begin{aligned}
\text { - if } x_{1} \neq 0: \quad \log \left(\frac{1}{d_{X}(x, y)}\right) & \approx \log \left(\frac{1}{\left|x_{1}-y_{1}\right|+\sqrt{\left|x_{1}\right|^{2}+\left|x_{2}-y_{2}\right|}-\left|x_{1}\right|}\right) \\
& \approx x_{x}-\frac{1}{2} \log \left(\frac{1}{2}-\frac{x_{1} y_{1}}{\sqrt{\left.\left(x_{1}^{2}+y_{1}^{2}\right)^{2}+4 x_{2}-y_{2}\right)^{2}}}\right)
\end{aligned}
$$

- Theorem 6.3: Firstly we consider the estimate in (6.4). At first glance, Theorem 6.5 does not seem to match with the product appearing in (6.4). However, the peculiarity of the latter is to provide an estimate which is uniform as both $x$ and $y$ vary (in some compact set). We show that a suitable choice of variable $x$ 's and $y$ 's confirm the product behavior of $\Gamma$. Indeed, let us take

$$
x(\epsilon):=\left(\epsilon, 2 \epsilon^{4}\right) \quad \text { and } \quad y(\epsilon):=\left(\epsilon, \epsilon^{4}\right) .
$$

As $\epsilon \rightarrow 0^{+}$, (6.21) gives (on account of (6.22) )

$$
\Gamma(x(\epsilon) ; y(\epsilon))=\frac{\gamma_{0}}{\sqrt[4]{\epsilon^{4}+\epsilon^{8}}} \cdot \mathrm{K}\left(\frac{1}{2}+\frac{1}{2 \sqrt{1+\epsilon^{4}}}\right) \sim \frac{2 c}{\epsilon} \log \left(\frac{1}{\epsilon}\right)=: h(\epsilon) .
$$

On the other hand, due to (6.18), we have

$$
d_{X}(x(\epsilon), y(\epsilon)) \approx \sqrt{\epsilon^{2}+\epsilon^{4}}-\epsilon \sim \frac{\epsilon^{3}}{2}, \quad \text { as } \epsilon \rightarrow 0^{+} .
$$

Thus, the estimate (6.24) together with (6.19) give

$$
\frac{d_{X}(x(\epsilon), y(\epsilon))^{2}}{\left|B_{X}\left(x(\epsilon), d_{X}(x(\epsilon), y(\epsilon))\right)\right|} \cdot \log \left(\frac{1}{d_{X}(x(\epsilon), y(\epsilon))}\right) \approx \frac{1}{\epsilon+\epsilon^{3}} \cdot \log \left(\frac{1}{\epsilon^{3}}\right) \approx \frac{\log (1 / \epsilon)}{\epsilon} .
$$

Since the latter term is $\approx h(\epsilon)$, these computations fully confirm (6.4) in Theorem 6.3.

Secondly we consider the estimate in (6.5): we observe that the function bounding $\Gamma$ from below in (6.5) cannot be of the same product form as in (6.4). Indeed, suitable choices of $x$ 's and $y$ 's show either logarithmic or power-like behaviors; for example, one can easily recognize what follows (starting from the explicit formula (6.21)):

$$
\begin{aligned}
& \Gamma((1,2 \epsilon) ;(1, \epsilon))=\frac{\gamma_{0} \sqrt{2}}{\sqrt[4]{4+4 \epsilon^{2}}} \cdot \mathrm{K}\left(\frac{1}{2}+\frac{1}{\sqrt{4+4 \epsilon^{2}}}\right) \sim \gamma_{0} \ln \left(\frac{1}{\epsilon}\right), \quad \text { as } \epsilon \rightarrow 0^{+} ; \\
& \Gamma((0, \epsilon) ;(\epsilon, \epsilon))=\frac{\gamma_{0} \sqrt{2} \mathrm{~K}(1 / 2)}{\epsilon} \quad \text { for every } \epsilon>0 .
\end{aligned}
$$

For completeness, we provide a full argument proving $(6.22)$ in the next remark.

Remark 6.8. After the change of variable $t=\sin \theta$, one has

We rewrite the integrand function as

$$
\mathrm{K}(m)=\int_{0}^{1} \frac{1}{\sqrt{1-m t^{2}}} \frac{\mathrm{d} t}{\sqrt{1-t^{2}}} .
$$

$$
\frac{f(t, m)}{2 \sqrt{1-t} \sqrt{1-\sqrt{m} t}}, \quad \text { where } \quad f(t, m)=\frac{2}{\sqrt{1+t} \sqrt{1+\sqrt{m} t}} .
$$

\footnotetext{
${ }^{6}$ One can show that both following functions have the same Taylor expansion as $y \rightarrow x$ :$$
\left|x_{1}-y_{1}\right|+\sqrt{\left|x_{1}\right|^{2}+\left|x_{2}-y_{2}\right|}-\left|x_{1}\right| \approx\left(\frac{1}{2}-\frac{x_{1} y_{1}}{\sqrt{\left(x_{1}^{2}+y_{1}^{2}\right)^{2}+4\left(x_{2}-y_{2}\right)^{2}}}\right)^{1 / 2} .
$$ 
As $f(1,1)=1$, for any fixed $\epsilon>0$ we choose $0<m(\epsilon), \delta(\epsilon) \ll 1$ such that $1-\epsilon \leq f(t, m) \leq 1-\epsilon$, whenever $1-m(\epsilon) \leq m \leq 1$ and $1-\delta(\epsilon) \leq t \leq 1$. We have

$$
\mathrm{K}(m)=\int_{0}^{1-\delta(\epsilon)} \frac{1}{\sqrt{1-m t^{2}}} \frac{\mathrm{d} t}{\sqrt{1-t^{2}}}+\int_{1-\delta(\epsilon)}^{1} \frac{f(t, m)}{2 \sqrt{1-t} \sqrt{1-\sqrt{m} t}} \mathrm{~d} t=: A(m, \epsilon)+B(m, \epsilon) .
$$

The first summand satisfies

$$
0 \leq A(m, \epsilon) \leq \int_{0}^{1-\delta(\epsilon)} \frac{\mathrm{d} t}{1-t^{2}}=: A^{\prime}(\epsilon), \quad \forall m \in[0,1] .
$$

Due to the estimate on $f(t, m)$, the second summand satisfies

$$
(1-\epsilon) \int_{1-\delta(\epsilon)}^{1} \frac{\mathrm{d} t}{2 \sqrt{1-t} \sqrt{1-\sqrt{m} t}} \leq B(m, \epsilon) \leq(1+\epsilon) \int_{1-\delta(\epsilon)}^{1} \frac{\mathrm{d} t}{2 \sqrt{1-t} \sqrt{1-\sqrt{m} t}},
$$

whenever $1-m(\epsilon) \leq m \leq 1$. A direct computation gives

$$
\begin{aligned}
\int_{1-\delta(\epsilon)}^{1} \frac{\mathrm{d} t}{2 \sqrt{1-t} \sqrt{1-\sqrt{m} t}} & =\frac{1}{\sqrt[4]{m}}\{\ln (\sqrt{1-(1-\delta(\epsilon)) \sqrt{m}}+\sqrt{\delta(\epsilon)} \sqrt[4]{m})-\ln \sqrt{1-\sqrt{m}}\} \\
=: & C(m, \epsilon)-\frac{\ln \sqrt{1-\sqrt{m}}}{\sqrt[4]{m}}=: C(m, \epsilon)+q(m),
\end{aligned}
$$

where $C(m, \epsilon)$ satisfies (uniformly for $m \in\left[\frac{1}{16}, 1\right]$ )

$$
\ln \left(\frac{3}{2} \sqrt{\delta(\epsilon)}\right)=: C^{\prime}(\epsilon) \leq C(m, \epsilon) \leq C^{\prime \prime}(\epsilon):=2 \ln \left(\frac{1}{2} \sqrt{3+\delta(\epsilon)}+\sqrt{\delta(\epsilon)}\right)
$$

Gathering together all the estimates on $A, B, C$ we get

$$
\frac{\mathrm{K}(m)}{q(m)}=\frac{A(m, \epsilon)}{q(m)}+\frac{B(m, \epsilon)}{q(m)},
$$

where (since $q(m) \longrightarrow \infty$ as $m \rightarrow 1^{-}$)

$$
0 \leq \frac{A(m, \epsilon)}{q(m)} \leq \frac{A^{\prime}(\epsilon)}{q(m)} \longrightarrow 0 \quad \text { as } m \rightarrow 1^{-},
$$

and moreover (if $1-m(\epsilon) \leq m \leq 1$ )

$$
(1-\epsilon)\left(\frac{C(m, \epsilon)}{q(m)}+1\right) \leq \frac{B(m, \epsilon)}{q(m)} \leq(1+\epsilon)\left(\frac{C(m, \epsilon)}{q(m)}+1\right),
$$

where

$$
\frac{C^{\prime}(\epsilon)}{q(m)} \leq \frac{C(m, \epsilon)}{q(m)} \leq \frac{C^{\prime \prime}(\epsilon)}{q(m)}, \quad \text { and } \quad \frac{C^{\prime}(\epsilon)}{q(m)}, \frac{C^{\prime \prime}(\epsilon)}{q(m)} \longrightarrow 0 \quad \text { as } m \rightarrow 1^{-} .
$$

The above computations show that (for some $0<m^{\prime \prime}(\epsilon) \ll 1$ )

$$
(1-\epsilon)^{2} \leq \frac{\mathrm{K}(m)}{q(m)} \leq \epsilon+(1+\epsilon)^{2}, \quad \text { whenever } 1-m^{\prime \prime}(\epsilon) \leq m \leq 1 .
$$

This proves that $\frac{\mathrm{K}(m)}{q(m)} \longrightarrow 1$, as $m \rightarrow 1^{-}$, that is, $\mathrm{K}(m) \sim q(m)$ as $m \rightarrow 1^{-}$. On the other hand,

$$
q(m)=-\frac{\ln \sqrt{1-\sqrt{m}}}{\sqrt[4]{m}} \sim-\ln \sqrt{1-\sqrt{m}} \sim-\frac{1}{2} \ln (1-m) \quad \text { as } m \rightarrow 1^{-} .
$$

This ends the proof of (6.8). 


\section{Applications to potential theory}

We let $\mathcal{L}:=X_{1}^{2}+\cdots+X_{m}^{2}$, where $X_{1}, \ldots, X_{m}$ are smooth vector fields satisfying our assumptions (H.1)-to-(H.3) in Section 2, As usual, $\Gamma$ is its global fundamental solution as in Theorem A. The assumption $q>2$ (see (1.2)) is still valid throughout the section. In what follows, we say that a function $u$ is $\mathcal{L}$-harmonic on an open set $\Omega \subseteq \mathbb{R}^{n}$ if $u \in C^{2}(\Omega)$ and $\mathcal{L} u=0$ in $\Omega$.

The aim of this section is to show that our operator $\mathcal{L}$ enjoys all the axioms in [1, 2, 4, 12, thus allowing us to derive the following potential-theoretic results: several characterizations of the cone of the $\mathcal{L}$-subharmonic functions in 12 ; the topological properties of the sheaf of the $\mathcal{L}$-harmonic functions in [2]; the rigidity inverse-mean-value theorem in [1]; the non-homogeneous and invariant Harnack inequality for $\mathcal{L}$ in $[4]$.

To this end, all that we have to do is to check that the axiomatic assumptions in [1, 2, 4, 12 are fulfilled in our case; this is contained in the following list (A.1)-to-(A.9). We are not interested in describing how any single axiom is involved in obtaining Theorems 7.2 to-7.7 the interested reader will find details in the mentioned papers. We only confine ourselves to a few remarks: the verification of axiom (A.8) is quite delicate, and it requires the upper estimates of $\Gamma$ and of its first derivatives given in Theorem 4.1, together with the lower estimates of $\Gamma$ in Theorem 5.1 , the validity of a global Poincaré inequality as in axiom (A.9) is obviously of independent interest.

(A.1) As a consequence of our assumption (H.1), all the $X_{i}$ 's have null divergence; hence, $\mathcal{L}$ is a purely divergence-form operator, that is,

$$
\mathcal{L}=\sum_{i, j=1}^{n} \partial_{x_{i}}\left(a_{i, j}(x) \partial_{x_{j}}\right)=\operatorname{div}(A(x) \nabla),
$$

where $A(x)=S(x) \cdot S(x)^{T}$ and $S(x)$ is the $n \times m$ matrix whose $i$-th column is $X_{i}(x)$.

$($ A.2) $\mathcal{L}$ admits a direction of strict ellipticity; in other words, there exists $i \in\{1, \ldots, n\}$ such that $a_{i, i}$ is a positive constant. This is another consequence of the $\delta_{\lambda}$-homogeneity of $X_{1}, \ldots, X_{m}$ (see e.g., [13, §1.3.1]).

(A.3) $\mathcal{L}$ is $C^{\infty}$-hypoelliptic on every open subset $\Omega$ of $\mathbb{R}^{n}$ : this a consequence of the Hörmander Hypoellipticity Theorem, which we are allowed to invoke owing to assumption (H.2). As a matter of fact, our $\mathcal{L}$ has polynomial (hence, real analytic) coefficient functions (due to our homogeneity assumption), so that hypoellipticity is indeed equivalent to (H.2); see, e.g., [18.

(A.4) $\mathcal{L}$ satisfies the so-called Regularity Axiom, namely, there exists a basis $\mathcal{B}$ for the Euclidean topology of $\mathbb{R}^{n}$, whose elements are bounded open sets $\Omega$, such that the Dirichlet problem

$$
\begin{cases}\mathcal{L} u=0 & \text { on } \Omega \\ u=\varphi & \text { on } \partial \Omega\end{cases}
$$

admits a (unique) solution $u \in C^{2}(\Omega) \cap C(\bar{\Omega})$, for every $\varphi \in C(\partial \Omega)$. This is true of any sums of squares of Hörmander vector fields, as proved by Bony in the seminal paper [14].

(A.5) $\mathcal{L}$ satisfies the so-called Doob Convergence Axiom: if $\left\{u_{k}\right\}_{k}$ is an increasing sequence of $\mathcal{L}$-harmonic functions on an open set $\Omega \subseteq \mathbb{R}^{n}$, then $u:=\sup _{k} u_{k}$ is $\mathcal{L}$-harmonic in $\Omega$, provided that $u$ is finite in a dense subset of $\Omega$. This is a consequence of the Harnack inequality proved by Bony in 14] for Hörmander sums of squares (see also [3] ).

(A.6) $\mathcal{L}$ is endowed with a global fundamental solution $\Gamma$, with the following basic properties: $\Gamma>0$ out of the diagonal; $\Gamma$ is locally integrable in $\mathbb{R}^{n} \times \mathbb{R}^{n} ; \Gamma(x ; \cdot)$ vanishes at infinity, for every fixed $x$. This is contained in our Theorem $\mathrm{A}$.

(A.7) $\Gamma(x ; \cdot)$ has a pole at every fixed $x$. This is contained in Theorems 5.1 and 6.3

We are now ready to derive from axioms (A.1)-to-(A.7) plenty of potential theoretic results for $\mathcal{L}$ (see Theorems 7.2 and $[7.3$ ). In order to do this, as a crucial tool of this section, we replace the geometry of the CC-balls $B_{X}(x, r)$ with the superlevel sets of $\Gamma(x ; \cdot)$, that is,

$$
\Omega_{r}(x):=\left\{y \in \mathbb{R}^{n}: \Gamma(x ; y)>1 / r\right\} \cup\{x\}, \quad x \in \mathbb{R}^{n}, r>0 .
$$


One of the greatest advantages of $\Omega_{r}(x)$ is that its boundary

$$
\partial \Omega_{r}(x)=\left\{y \in \mathbb{R}^{n}: \Gamma(x ; y)=1 / r\right\} .
$$

is a smooth manifold of dimension $n-1$, at least for almost every $r$ (by Sard's Lemma).

For simplicity, we tacitly agree that all the statements of this section involving $\partial \Omega_{r}(x)$ hold for those $r>0$ for which $\partial \Omega_{r}(x)$ is smooth (hence, for almost every $r$ ). Let now $x \in \mathbb{R}^{n}$, and let us consider the functions, defined for $y \neq x$,

$$
\Gamma_{x}(y):=\Gamma(x ; y), \quad \mathcal{K}_{x}(y):=\frac{\sum_{j=1}^{m}\left|X_{j} \Gamma_{x}(y)\right|^{2}}{\left|\nabla \Gamma_{x}(y)\right|} .
$$

Let $u$ be upper semicontinuous on $\Omega$. For every fixed $\alpha>0$, every $x \in \mathbb{R}^{n}$ and $r>0$ such that $\overline{\Omega_{r}(x)} \subset \Omega$, we introduce the following mean-value integral operators (here $H^{n-1}$ is the standard $(n-1)$-dimensional Hausdorff measure in $\left.\mathbb{R}^{n}\right)$ :

$$
m_{r}(u)(x)=\int_{\partial \Omega_{r}(x)} u(y) \mathcal{K}_{x}(y) \mathrm{d} H^{n-1}(y), \quad M_{r}(u)(x)=\frac{\alpha+1}{r^{\alpha+1}} \int_{0}^{r} \rho^{\alpha} m_{\rho}(u)(x) \mathrm{d} \rho .
$$

An alternative representation of $M_{r}$ is the following one:

$$
M_{r}(u)(x)=\frac{\alpha+1}{r^{\alpha+1}} \int_{\Omega_{r}(x)} u(y) K_{x}^{\alpha}(y) \mathrm{d} y, \quad \text { where } \quad K_{x}^{\alpha}(y):=\frac{\sum_{j=1}^{m}\left|X_{j} \Gamma_{x}(y)\right|^{2}}{\Gamma_{x}^{2+\alpha}(y)} .
$$

Furthermore, for every $x \in \mathbb{R}^{n}$ and every $r>0$, we set

$$
\begin{aligned}
& q_{r}(x)=\int_{\Omega_{r}(x)}\left(\Gamma_{x}(y)-\frac{1}{r}\right) \mathrm{d} y, \quad Q_{r}(x)=\frac{\alpha+1}{r^{\alpha+1}} \int_{0}^{r} \rho^{\alpha} q_{\rho}(x) \mathrm{d} \rho, \\
& \omega_{r}(x)=\frac{1}{\alpha r^{\alpha+1}} \int_{\Omega_{r}(x)}\left(r^{\alpha}-\Gamma_{x}^{-\alpha}(y)\right) \mathrm{d} y .
\end{aligned}
$$

Remark 7.1. The above operators $m_{r}, M_{r}$ permit to obtain the following analogs of the classical Gauss-Green formulas for Laplace's operator (see, e.g., [12]):

$$
\begin{aligned}
& u(x)=m_{r}(u)(x)-\int_{\Omega_{r}(x)}\left(\Gamma_{x}(y)-\frac{1}{r}\right) \mathcal{L} u(y) \mathrm{d} y, \\
& u(x)=M_{r}(u)(x)-\frac{\alpha+1}{r^{\alpha+1}} \int_{0}^{r} \rho^{\alpha}\left(\int_{\Omega_{\rho}(x)}\left(\Gamma_{x}(y)-\frac{1}{\rho}\right) \mathcal{L} u(y) \mathrm{d} y\right) \mathrm{d} \rho,
\end{aligned}
$$

holding true for every function $u$ of class $C^{2}$ on an open set containing $\overline{\Omega_{r}(x)}$.

From now on, $\Omega$ will denote an open set. An upper semicontinuous function $u: \Omega \rightarrow[-\infty, \infty)$ is called $\mathcal{L}$-subharmonic in $\Omega$ if $u \not \equiv-\infty$ on every component of $\Omega$, and the following holds: for every bounded open set $V \subset \bar{V} \subset \Omega$ and for every $\mathcal{L}$-harmonic function $h \in C^{2}(V) \cap C(\bar{V})$ such that $u \leq h$ on $\partial V$, one has $u \leq h$ in $V$.

For simplicity, the following result, providing characterizations of $\mathcal{L}$-subharmonicity, is stated for continuous functions $u$, but it also holds for a u.s.c. function, with minor modification (see [12]); this is a consequence of axioms (A.1)-to-(A.7).

Theorem 7.2. Suppose that $u \in C(\Omega)$ and let $q_{r}, Q_{r}, \omega_{r}$ be as in (7.2). Let also

$$
R(x):=\sup \left\{r>0: \Omega_{r}(x) \subseteq \Omega\right\} .
$$

Then, the following conditions are equivalent:

(1) $u$ is $\mathcal{L}$-subharmonic in $\Omega$.

(2) $\mathcal{L} u \geq 0$ in the weak sense of distributions.

(3) $u(x) \leq m_{r}(u)(x)$, for every $x \in \Omega$ and $r \in(0, R(x))$.

(4) $u(x) \leq M_{r}(u)(x)$, for every $x \in \Omega$ and $r \in(0, R(x))$.

(5) $M_{r}(u)(x) \leq m_{r}(u)(x)$, for every $x \in \Omega$ and $r \in(0, R(x))$.

(6) $r \mapsto m_{r}(u)(x)$ is monotone increasing on $(0, R(x))$, for every $x \in \Omega$.

(7) $r \mapsto M_{r}(u)(x)$ is monotone increasing on $(0, R(x))$, for every $x \in \Omega$. 
(8) For every $x \in \Omega$ it holds that

$$
\limsup _{r \rightarrow 0} \frac{m_{r}(u)(x)-u(x)}{q_{r}(x)} \geq 0 .
$$

(9) For every $x \in \Omega$ it holds that

$$
\limsup _{r \rightarrow 0} \frac{M_{r}(u)(x)-u(x)}{Q_{r}(x)} \geq 0 .
$$

(10) For every $x \in \Omega$ it holds that

$$
\liminf _{r \rightarrow 0} \frac{m_{r}(u)(x)-M_{r}(u)(x)}{\omega_{r}(x)} \geq 0 .
$$

As for the sheaf of the $\mathcal{L}$-harmonic functions, we have the following result, obtained by collecting the axiomatic investigations in [2]: this provides a characterization of $\mathcal{L}$-harmonicity, together with some topological properties of the vector space of the $\mathcal{L}$-harmonic functions on an open set. This is again a consequence of axioms (A.1)-to-(A.7).

Theorem 7.3. Suppose that $u \in C(\Omega)$. Then, the following conditions are equivalent:

(1) $u$ is $C^{\infty}$ and is $\mathcal{L}$-harmonic in $\Omega$.

(2) $u(x)=m_{r}(u)(x)$, for every $x \in \Omega$ and $r \in(0, R(x))$.

(3) $u(x)=M_{r}(u)(x)$, for every $x \in \Omega$ and $r \in(0, R(x))$.

Furthermore, we have the following compactness Montel-type result for $\mathcal{L}$. Let $\mathcal{F}$ be a family of $\mathcal{L}$-harmonic functions on $\Omega$ which is locally bounded, that is,

$$
\sup _{f \in \mathcal{F}}\left(\sup _{K}|f|\right)<\infty, \quad \text { for every compact set } K \subset \Omega \text {. }
$$

Then $\mathcal{F}$ is a normal family, that is, for every sequence $\left\{f_{n}\right\}_{n}$ in $\mathcal{F}$, there exists a subsequence of $\left\{f_{n}\right\}_{n}$ which is uniformly convergent on the compact subsets of $\Omega$.

Finally, the set of the $\mathcal{L}$-harmonic functions in $\Omega$ endowed with the $L_{\text {loc }}^{1}$-topology inherited from $C(\Omega)$ (or, equivalently, endowed with the $L_{\mathrm{loc}}^{\infty}$-topology) is a Heine-Borel topological vector space.

In order to have the next Theorem 7.5, we need to verify another axiom:

(A.8) There exists $\alpha>0$ such that the sequence of functions

$$
f_{k}(x):=\int_{\Omega_{1 / k}(x)} \Gamma(x ; y) K_{0}^{\alpha}(y) \mathrm{d} y
$$

vanishes as $k \rightarrow \infty$, uniformly in $x$ (when $x$ lies in a compact set).

Let us prove that (A.8) is fulfilled in our case, when $n>2$, with the choice $\alpha>2 /(q-2)$. To begin with, by the very definition of $K_{0}^{\alpha}$, and owing to Theorems 4.1 and 5.1, one easily proves

$$
K_{0}^{\alpha}(y) \leq C \frac{\left|B_{X}\left(0, d_{X}(0, y)\right)\right|^{\alpha}}{d_{X}(0, y)^{2+2 \alpha}} \stackrel{(2.2)}{=}\left|B_{X}(0,1)\right|^{\alpha} d_{X}(0, y)^{\alpha(q-2)-2} .
$$

Since, by assumption $\alpha(q-2)-2>0$, we conclude that $K_{0}^{\alpha}$ is bounded on any compact set. Then, if $x$ lies in a fixed compact set $F \subseteq \mathbb{R}^{n}$ and $y \in \Omega_{1 / k}(x)$, one has

$$
\sup _{y \in \Omega_{1 / k}(x)} K_{0}^{\alpha}(y) \leq M(F), \quad \text { for every } x \in F \text { and every } k \in \mathbb{N} .
$$

Thus (A.8) is fulfilled if we show that

$$
\int_{\Omega_{1 / k}(x)} \Gamma(x ; y) \mathrm{d} y \longrightarrow 0 \text { as } k \rightarrow \infty \text {, uniformly for } x \in F .
$$

To prove this, we first remark that, due to Theorem B, if $y \in \Omega_{r}(x)$ one has

$$
\begin{aligned}
1 / r<\Gamma(x ; y) & \stackrel{\sqrt{4.1}}{\leq} C \frac{d_{X}(x, y)^{2}}{\left|B_{X}\left(x, d_{X}(x, y)\right)\right|} \\
& \stackrel{(2.7)}{\leq} \frac{C}{\gamma_{1}}\left(\sum_{h=n}^{q} f_{h}(x) d_{X}(x, y)^{h-2}\right)^{-1} \leq \frac{C}{f_{q} \gamma_{1}} d_{X}(x, y)^{2-q}
\end{aligned}
$$


This shows that (with the structural choice $\theta^{q-2}:=C /\left(f_{q} \gamma_{1}\right)$ )

$$
\Omega_{r}(x) \subseteq B_{X}\left(x, \theta r^{1 /(q-2)}\right), \quad \text { for any } x \in \mathbb{R}^{n} \text { and any } r>0 .
$$

Due to (7.4), we derive (7.3) from Theorem 4.1 and the following result (with $p=2$ ).

Lemma 7.4. For every $p>0$, every $x \in \mathbb{R}^{n}$ and every $r>0$, one has

$$
\int_{B_{X}(x, r)} \frac{d_{X}(x, y)^{p}}{\left|B_{X}\left(x, d_{X}(x, y)\right)\right|} \mathrm{d} y \leq c_{p} r^{p}, \quad \text { where } c_{p}=C_{d} \frac{2^{p}}{2^{p}-1},
$$

and $C_{d}$ is the doubling constant in (2.3).

Proof. We have the following argument, only based on the doubling inequality:

$$
\begin{aligned}
& \int_{B_{X}(x, r)} \frac{d_{X}(x, y)^{p}}{\left|B_{X}\left(x, d_{X}(x, y)\right)\right|} \mathrm{d} y=\sum_{k=0}^{\infty} \int_{\frac{r}{2^{k+1}} \leq d_{X}(x, y)<\frac{r}{2^{k}}} \frac{d_{X}(x, y)^{p}}{\left|B_{X}\left(x, d_{X}(x, y)\right)\right|} \mathrm{d} y \\
& \leq \sum_{k=0}^{\infty} \frac{\left|B_{X}\left(x, r / 2^{k}\right)\right|}{\left|B_{X}\left(x, r / 2^{k+1}\right)\right|}\left(\frac{r}{2^{k}}\right)^{p} \leq C_{d} r^{p} \sum_{k=0}^{\infty}\left(\frac{1}{2^{p}}\right)^{k}=c_{p} r^{p} .
\end{aligned}
$$

This completes the proof of (7.5).

Thanks to the validity of axioms (A.1)-to-(A.8) in our framework (with $n>2$ ), we have the following rigidity-type result for $\mathcal{L}$ (also referred to as an inverse-mean-value theorem); see [1].

Theorem 7.5. Let $n>2$. We choose $\alpha>2 /(q-2)$ and, with reference to (7.1), we consider the measure $\mathrm{d} \mu(y)=K_{0}^{\alpha}(y) \mathrm{d} y$. Then (3) in Theorem 7.3 gives

$$
u(0)=\frac{1}{\mu\left(\Omega_{r}(0)\right)} \int_{\Omega_{r}(0)} u(y) \mathrm{d} \mu(y),
$$

for every $u$ which is $\mathcal{L}$-harmonic on a neighborhood of $\overline{\Omega_{r}(0)}$.

Conversely, we have the following characterization of the superlevel sets $\Omega_{r}(0)$ of $\Gamma$. Suppose that $D$ is a bounded open neighborhood of 0 such that

$$
u(0)=\frac{1}{\mu(D)} \int_{D} u(y) \mathrm{d} \mu(y),
$$

for every $u$ which is $\mathcal{L}$-harmonic and $\mu$-integrable on $D$. Then $D=\Omega_{r}(0)$ for some $r>0$.

More precisely, it suffices to suppose that (7.6) holds for the family of $\mathcal{L}$-harmonic functions

$$
\left\{D \ni y \mapsto \Gamma_{x}(y)\right\}_{x \notin D} .
$$

As a last application, in order to have the following Harnack Theorem 7.7 we need to check the validity of the next axiom in our framework:

(A.9) The global doubling inequality (2.3) holds, together with the following result:

Theorem 7.6 (Global Poincaré inequality). Let $X=\left\{X_{1}, \ldots, X_{m}\right\}$ satisfy axioms (H.1) and (H.2). There exists a constant $C_{P}>0$ such that, for every $x \in \mathbb{R}^{n}, r>0$ and every $u$ which is $C^{1}$ in a neighborhood of $B_{X}(x, 2 r)$, one has

$$
f_{B_{X}(x, r)}\left|u(y)-u_{B_{X}(x, r)}\right| \mathrm{d} y \leq C_{P} r f_{B_{X}(x, 2 r)}|X u(y)| \mathrm{d} y .
$$

As usual we have set $|X u|:=\sqrt{\sum_{j=1}^{m}\left|X_{j} u\right|^{2}}$, and (if $B$ is any $d_{X}$-ball)

$$
u_{B}:=f_{B} u:=\frac{1}{|B|} \int_{B} u(y) \mathrm{d} y .
$$

We have already proved, via a homogeneity argument, that the global doubling inequality (2.3) holds in our case. The validity of (7.7) follows likewise: indeed, from the results in 23. one knows of the existence of a neighborhood $U_{0}$ of 0 , a constant $P_{0}>0$ and $r_{0}>0$ such that

$$
\int_{B_{X}(\xi, \rho)}\left|v(y)-v_{B_{X}(\xi, \rho)}\right| \mathrm{d} y \leq P_{0} \rho \int_{B_{X}(\xi, 2 \rho)}|X v(y)| \mathrm{d} y,
$$


for every $\xi \in U_{0}, \rho \in\left[0, r_{0}\right]$ and $v \in C^{1}\left(\overline{B_{2 \rho}(\xi)}\right)$. Let $x \in \mathbb{R}^{n}, r>0$ and $u \in C^{1}\left(\overline{B_{X}(x, 2 r)}\right)$; there certainly exists $0<\lambda \ll 1$ such that $\delta_{\lambda}(x) \in U_{0}$ and $\lambda r<r_{0}$. It is easy to check (via (2.2)) that

$$
f_{B_{X}(x, r)} u=f_{B_{X}\left(\delta_{\lambda}(x), \lambda r\right)} v, \quad \text { where } v=u \circ \delta_{1 / \lambda} .
$$

The change of variable $y=\delta_{1 / \lambda}(z)$ (applied twice) proves the following computation:

$$
\begin{aligned}
& \int_{B_{X}(x, r)}\left|u(y)-u_{B_{X}(x, r)}\right| \mathrm{d} y=\lambda^{-q} \int_{B_{X}\left(\delta_{\lambda}(x), \lambda r\right)}\left|v-v_{B_{X}\left(\delta_{\lambda}(x), \lambda r\right)}\right| \mathrm{d} z \\
& \stackrel{(7.8)}{\leq} \lambda^{-q} P_{0} \lambda r \int_{B_{X}\left(\delta_{\lambda}(x), 2 \lambda r\right)}|X v| \mathrm{d} z=\lambda^{-q} P_{0} \lambda r \int_{B_{X}\left(\delta_{\lambda}(x), 2 \lambda r\right)} \frac{1}{\lambda}|X u|\left(\delta_{1 / \lambda}(z)\right) \mathrm{d} z \\
& =P_{0} r \int_{B_{X}(x, 2 r)}|X u|(y) \mathrm{d} y .
\end{aligned}
$$

Summing up, we have proved that (for general $x, r, u$ as above)

$$
\int_{B_{X}(x, r)}\left|u(y)-u_{B_{X}(x, r)}\right| \mathrm{d} y \leq P_{0} r \int_{B_{X}(x, 2 r)}|X u| \mathrm{d} y .
$$

If we divide both sides by $\left|B_{X}(x, r)\right|$ and if we apply the global doubling inequality (2.3), we get at once (7.7) of axiom (A.9), with $C_{P}=P_{0} C_{d}$, where $C_{d}$ is as in (2.3).

Thanks to the axiomatic theory carried out in 4, due to axioms (A.1) and (A.9) only (plus our hypotheses (H.1) and (H.2)), we can deduce the following non-homogeneous and invariant Harnack inequality for $\mathcal{L}$. For simplicity, we state it for classical solutions, but it also holds for $W_{\text {loc }}^{1}$-weak solutions (in the sense of the Sobolev spaces associated with $X_{1}, \ldots, X_{m}$ ); see [4].

Theorem 7.7 (Global scale-invariant Harnack inequality). Let $g \in L^{p}(\Omega)$, with $p>\frac{q}{2}$. There exists a structural constant $C_{p}>0$ such that, for every ball $B_{X}(x, R)$ satisfying $\overline{B_{X}(x, 4 R)} \subset \Omega$, one has

$$
\sup _{B_{X}(x, R)} u \leq C\left\{\inf _{B_{X}(x, R)} u+R^{2}\left(f_{B_{X}(x, 4 R)}|g|^{p}\right)^{1 / p}\right\},
$$

for any nonnegative solution $u$ of $\mathcal{L} u=g$ in $\Omega$.

The invariance of the above Harnack inequality proves at once the classical Liouville theorem for $\mathcal{L}:$ if $\mathcal{L} v=0$ on $\mathbb{R}^{n}$ and if $\inf _{\mathbb{R}^{n}} v>-\infty$, then $v$ is constant.

\section{Applications to Singular Integrals}

Starting with the representation formula

$$
-\phi(x)=\int_{\mathbb{R}^{n}} \Gamma(x ; y) \mathcal{L} \phi(y) \mathrm{d} y,
$$

for every $\phi \in C_{0}^{\infty}\left(\mathbb{R}^{n}\right)$ and $x \in \mathbb{R}^{n}$ (see Theorem A), it is reasonable to expect that one can prove a representation formula for second order derivatives of $\phi$, involving a singular integral, of the kind:

$$
X_{i} X_{j} \phi(x)=\mathrm{PV} \int_{\mathbb{R}^{n}} X_{i}^{x} X_{j}^{x} \Gamma(x ; y)(-\mathcal{L} \phi)(y) \mathrm{d} y+c_{i, j}(x) \mathcal{L} \phi(x),
$$

valid for any $i, j=1,2, \ldots, m$, for suitable bounded functions $c_{i, j}$. This means that it is worthwhile studying the properties of the singular kernel

$$
\left.k(x, y):=X_{i}^{x} X_{j}^{x} \Gamma(x ; y) \quad \text { (for fixed } i, j \in\{1, \ldots, m\}\right) .
$$

Actually, in view of the global doubling condition established in (2.3), the space $\mathbb{R}^{n}$, endowed with the CC-distance of the vector fields $X_{1}, \ldots, X_{m}$ and the Lebesgue measure, is (globally) a space of homogeneous type in the sense of Coifman-Weiss [17. Hence, once we have established a suitable set of properties of this kernel, the continuity on $L^{p}\left(\mathbb{R}^{n}\right)(1<p<\infty)$ of the operator

$$
T: f \mapsto \mathrm{PV} \int_{\mathbb{R}^{n}} X_{i} X_{j} \Gamma(\cdot ; y) f(y) \mathrm{d} y
$$


should follow, hopefully, just through the application of some existing general abstract theory. This fact would provide global estimates in $W_{X}^{2, p}$ spaces for the operator $\mathcal{L}$.

In this section we shall prove some properties of the kernel $k(x, y)$ which are relevant to this aim. We shall not prove, here, a representation formula (8.1), nor shall we develop the subsequent theory which would give the alluded Sobolev estimates. Actually, this material would overburden the present paper, and will be the subject of a separate paper. We just point out that this technique can also be used to prove global Sobolev estimates for "nonvariational operators" of the kind

$$
L u=\sum_{i, j=1}^{m} a_{i, j}(x) X_{i} X_{j} u,
$$

where $\left(a_{i, j}\right)_{i, j}$ is a symmetric, uniformly positive matrix of bounded coefficient functions, possessing some minimal regularity.

For notational simplicity, in this section we write $d$ and $B$ instead of $d_{X}$ and $B_{X}$. The theory of singular integrals usually requires to check that both a kernel $k(x, y)$ and its adjoint $k(y, x)$ satisfy some pointwise and/or integral properties. The result we prove is the following:

Theorem 8.1 (Properties of the singular kernel $k(x, y)$ ). Let the assumptions and notation of the previous sections be in force and, for some fixed $i, j \in\{1,2, \ldots, m\}$, let

$$
k(x, y):=X_{i}^{x} X_{j}^{x} \Gamma(x ; y) \quad \text { for } x, y \in \mathbb{R}^{n}, x \neq y .
$$

There exist constants $A, B, C>0$ such that:

(i) for every $x, y \in \mathbb{R}^{n}(x \neq y)$ one has

$$
|k(x, y)|+|k(y, x)| \leq \frac{A}{|B(x, d(x, y))|} ;
$$

(ii) for every $x, x_{0}, y \in \mathbb{R}^{n}$ such that $d\left(x_{0}, y\right) \geq 2 d\left(x_{0}, x\right)>0$, it holds

$$
\left|k(x, y)-k\left(x_{0}, y\right)\right|+\left|k(y, x)-k\left(y, x_{0}\right)\right| \leq B \frac{d\left(x_{0}, x\right)}{d\left(x_{0}, y\right)} \cdot \frac{1}{\left|B\left(x_{0}, d\left(x_{0}, y\right)\right)\right|} ;
$$

(iii) for every $z \in \mathbb{R}^{n}$ and $0<r<R<\infty$, one has

$$
\left|\int_{\{r<d(z, y)<R\}} k(z, y) \mathrm{d} y\right|+\left|\int_{\{r<d(z, x)<R\}} k(x, z) \mathrm{d} x\right| \leq C .
$$

Inequalities (i)-(ii) are usually called the standard estimates of singular kernels, while (iii) is a kind of cancelation property, and is crucial in order to give sense to the principal value integral (8.1). These three properties are one of the possible sets of reasonable assumptions to prove that the singular integral operator with kernel $k$ is continuous on $L^{p}\left(\mathbb{R}^{n}\right)$ for every $p \in(1, \infty)$.

We explicitly note that the above theorem still holds in the case $n=2$ (since we are dealing with second order derivatives of $\Gamma$ ).

For the proof, we need to use the following:

Theorem 8.2 ("Lagrange Theorem"; 15, Theorem 1.55]). Let $X_{1}, \ldots, X_{m}$ be any system of Hörmander vector fields in a domain $\Omega \subseteq \mathbb{R}^{n}$, and let $f \in C^{1}\left(\overline{B\left(x_{0}, r\right)}\right)$, with $B\left(x_{0}, r\right) \Subset \Omega$. Then

$$
\left|f(x)-f\left(x_{0}\right)\right| \leq \sqrt{m} d\left(x, x_{0}\right) \sup _{B\left(x_{0}, r\right)}|X f|, \quad \text { for every } x \in B\left(x_{0}, r\right),
$$

where $|X f|=\sqrt{\sum_{i=1}^{m}\left|X_{i} f\right|^{2}}$.

For the sake of completeness, we give the proof of this known result.

Proof. Let $x \in B\left(x_{0}, r\right), d\left(x, x_{0}\right)=: \delta<r$. For every fixed $0<\varepsilon<r-\delta$, there exists a curve $\gamma(t)$ joining $x_{0}$ to $x$ such that

$$
\gamma^{\prime}(t)=\sum_{i=1}^{m} a_{i}(t) X_{i}(\gamma(t))
$$


with $\left|a_{i}(t)\right| \leq \delta+\varepsilon$ for any $i=1, \ldots, m$. Then

$$
f(x)-f\left(x_{0}\right)=\int_{0}^{1} \frac{\mathrm{d}}{\mathrm{d} t}(f(\gamma(t))) \mathrm{d} t=\int_{0}^{1} \sum_{i=1}^{m} a_{i}(t) X_{i} f(\gamma(t)) \mathrm{d} t .
$$

By definition of the CC-distance, all the points of the path $\gamma(t)$ are inside the ball $B\left(x_{0}, r\right)$, hence

$$
\left|f(x)-f\left(x_{0}\right)\right| \leq \sup _{t \in[0,1]} \sqrt{\sum_{i=1}^{m}\left|a_{i}(t)\right|^{2}} \cdot \sqrt{\sum_{i=1}^{m}\left|X_{i} f(\gamma(t))\right|^{2}} \leq \sqrt{m}(\delta+\varepsilon) \sup _{B_{X}\left(x_{0}, r\right)}|X f|,
$$

and, for the arbitrariness of $\varepsilon,(\underline{8.2})$ follows.

With this result at hand, let us pass to the

Proof of Theorem 8.1. By Theorem 4.1, we have

$$
|k(x, y)| \leq \frac{c}{|B(x, d(x, y))|}
$$

for every $x, y \in \mathbb{R}^{n}, x \neq y$. Since, as already noted in Remark 4.2 using the global doubling property,

$$
|B(x, d(x, y))| \approx|B(y, d(x, y))|,
$$

this implies (i). To prove (ii), we apply Theorem 8.2 to the following functions (here $y$ is fixed)

$$
f_{1}(x):=X_{i}^{x} X_{j}^{x} \Gamma(x ; y), \quad f_{2}(x):=X_{i}^{y} X_{j}^{y} \Gamma(y ; x),
$$

and we apply the upper bound on the third order derivatives of $\Gamma$ proved in Theorem 4.1. Next we take any $x, x_{0} \in \mathbb{R}^{n}$ such that $d\left(x_{0}, y\right) \geq 2 d\left(x_{0}, x\right)>0$. Letting

$$
r=\frac{3}{2} d\left(x, x_{0}\right)
$$

we have that $x \in B\left(x_{0}, r\right)$ and, by (8.2),

$$
\left|k(x, y)-k\left(x_{0}, y\right)\right|=\left|f_{1}(x)-f_{1}\left(x_{0}\right)\right| \leq \sqrt{m} d\left(x, x_{0}\right) \sup _{z \in B\left(x_{0}, r\right)}\left|X f_{1}(z)\right|,
$$

where, owing to Theorem 4.1, for any $k \in\{1, \ldots, m\}$ and any $z \in B\left(x_{0}, r\right)$,

$$
\left|X_{k} f_{1}(z)\right|=\left|X_{k}^{z} X_{i}^{z} X_{j}^{z} \Gamma(z, y)\right| \leq \frac{c}{d(z, y)|B(z, d(y, z))|}
$$

Since $z \in B\left(x_{0}, r\right)$ with $r=\frac{3}{2} d\left(x, x_{0}\right)$, and since $d\left(x_{0}, y\right) \geq 2 d\left(x_{0}, x\right)=\frac{4}{3} r$, we infer that

$$
\frac{1}{4} d\left(x_{0}, y\right) \leq d(z, y) \leq \frac{7}{4} d\left(x_{0}, y\right)
$$

hence, by the doubling condition and by (8.4), we get

$$
\sup _{z \in B\left(x_{0}, r\right)}\left|X f_{1}(z)\right| \leq \frac{c^{\prime}}{d\left(x_{0}, y\right)\left|B\left(x_{0}, d\left(x_{0}, y\right)\right)\right|}
$$

If we insert (8.5) in (8.3), we obtain

$$
\left|k(x, y)-k\left(x_{0}, y\right)\right| \leq \frac{c^{\prime \prime} d\left(x, x_{0}\right)}{d\left(x_{0}, y\right)\left|B\left(x_{0}, d\left(x_{0}, y\right)\right)\right|} .
$$

Analogously, one can reproduce this argument for

$$
\left|k(y, x)-k\left(y, x_{0}\right)\right|=\left|f_{2}(x)-f_{2}\left(x_{0}\right)\right|,
$$

this time the upper bound on the mixed third order derivatives $X_{k} f_{2}(z)=X_{k}^{z} X_{i}^{y} X_{j}^{y} \Gamma(y ; z)$ being needed. Thus we get

$$
\left|k(y, x)-k\left(y, x_{0}\right)\right| \leq \frac{c^{\prime \prime \prime} d\left(x, x_{0}\right)}{d\left(x_{0}, y\right)\left|B\left(x_{0}, d\left(x_{0}, y\right)\right)\right|} .
$$

Gathering together (8.6) and (8.7), we obtain the proof of (ii). 
The proof of (iii) is inspired to [16, Prop. 5.23]. By the representation formula (4.5), we have

$$
k(x, y)=X_{i}^{x} X_{j}^{x} \Gamma(x ; y)=\int_{\mathbb{R}^{p}}\left(\widetilde{X}_{i} \widetilde{X}_{j} \Gamma_{\mathbb{G}}\right)\left((y, 0)^{-1} *(x, \eta)\right) \mathrm{d} \eta .
$$

Then, thanks to Fubini's Theorem 7 we have

$$
\begin{aligned}
\int_{\{r<d(x, z)<R\}} k(x, z) \mathrm{d} x & =\int_{\mathbb{R}^{p}} \int_{\{r<d(x, z)<R\}}\left(\widetilde{X}_{i} \widetilde{X}_{j} \Gamma_{\mathbb{G}}\right)\left((z, 0)^{-1} *(x, \eta)\right) \mathrm{d} x \mathrm{~d} \eta \\
& =\int_{\left\{r<d_{\widetilde{X}}((z, 0),(x, \eta))<R\right\}}\left(\widetilde{X}_{i} \widetilde{X}_{j} \Gamma_{\mathbb{G}}\right)\left((z, 0)^{-1} *(x, \eta)\right) \mathrm{d} x \mathrm{~d} \eta \\
& +\int_{\left\{d_{\widetilde{X}}((z, 0),(x, \eta))>R, d(x, z)<R\right\}}\left(\widetilde{X}_{i} \widetilde{X}_{j} \Gamma_{\mathbb{G}}\right)\left((z, 0)^{-1} *(x, \eta)\right) \mathrm{d} x \mathrm{~d} \eta \\
& -\int_{\left\{d_{\widetilde{X}}((z, 0),(x, \eta))>r, d(x, z)<r\right\}}\left(\widetilde{X}_{i} \widetilde{X}_{j} \Gamma_{\mathbb{G}}\right)\left((z, 0)^{-1} *(x, \eta)\right) \mathrm{d} x \mathrm{~d} \eta \\
& =: C^{r, R}(z)+D^{R}(z)-D^{r}(z) .
\end{aligned}
$$

However, we know that the singular kernel $\widetilde{X}_{i} \widetilde{X}_{j} \Gamma_{\mathbb{G}}$ on the Carnot group $\mathbb{G}$ satisfies the vanishing property (see [19, Propositions 1.5, 1.8])

$$
\begin{aligned}
& \int_{\left\{r<d_{\widetilde{X}}((z, 0),(x, \eta))<R\right\}}\left(\widetilde{X}_{i} \widetilde{X}_{j} \Gamma_{\mathbb{G}}\right)\left((z, 0)^{-1} *(x, \eta)\right) \mathrm{d} x \mathrm{~d} \eta \\
& =\int_{\left\{r<d_{\widetilde{X}}((0,0),(x, \eta))<R\right\}}\left(\widetilde{X}_{i} \widetilde{X}_{j} \Gamma_{\mathbb{G}}\right)(x, \eta) \mathrm{d} x \mathrm{~d} \eta=0, \quad \text { for any } R>r>0,
\end{aligned}
$$

so that $C^{r, R}(z) \equiv 0$. Owing to Corollaries 4.11 and 6.2, we have

$$
\begin{aligned}
\left|D^{R}(z)\right| & \leq c \int_{\left\{d_{\widetilde{X}}((z, 0),(x, \eta))>R, d(x, z)<R\right\}} d_{\widetilde{X}}((z, 0),(x, \eta))^{-Q} \mathrm{~d} x \mathrm{~d} \eta \\
& \leq \frac{c}{R} \int_{\{d(x, z) \leq R\}}\left(\int_{\mathbb{R}^{p}} d_{\widetilde{X}}((x, 0),(z, \eta))^{-Q+1} \mathrm{~d} \eta\right) \mathrm{d} x \\
& \leq \frac{c}{R} \int_{\{d(x, z) \leq R\}} \frac{d(x, z)}{|B(x, d(x, z))|} \mathrm{d} z \leq \frac{c}{R} R=c,
\end{aligned}
$$

where in the last inequality we have exploited Lemma 7.4 (with $p=1$ ). The estimate of $\left|D^{r}(z)\right|$ is the very same, replacing $R$ with $r$. Summing up, we have proved that

$$
\left|\int_{\{r<d(x, z)<R\}} k(x, z) \mathrm{d} x\right| \leq\left|D^{R}(z)\right|+\left|D^{r}(z)\right| \leq c,
$$

with $c$ independent of $R, r, z$.

To prove the analogous bound on the integral with respect to $y$, let us write

$$
\int_{\{r<d(z, y)<R\}} k(z, y) \mathrm{d} y \stackrel{\sqrt{8.8}}{=} \int_{\mathbb{R}^{p}} \int_{\{r<d(z, y)<R\}}\left(\widetilde{X}_{i} \widetilde{X}_{j} \Gamma_{\mathbb{G}}\right)\left((y, 0)^{-1} *(z, \eta)\right) \mathrm{d} y \mathrm{~d} \eta ;
$$

via the change of variable $\eta=\Phi_{y, z}(\zeta)$ as in Lemma 4.5, the latter integral is equal to

$$
\begin{aligned}
& \int_{\mathbb{R}^{p}} \int_{\{r<d(z, y)<R\}}\left(\widetilde{X}_{i} \widetilde{X}_{j} \Gamma_{\mathbb{G}}\right)\left((y, u)^{-1} *(z, 0)\right) \mathrm{d} y \mathrm{~d} u \\
& =\int_{\mathbb{R}^{p}} \int_{\{r<d(z, y)<R\}}\left(\left(\widetilde{X}_{i} \widetilde{X}_{j} \Gamma_{\mathbb{G}}\right) \circ \iota\right)\left((z, 0)^{-1} *(y, u)\right) \mathrm{d} y \mathrm{~d} u .
\end{aligned}
$$

Then we can proceed as above, exploiting the fact that the kernel $\left(\widetilde{X}_{i} \widetilde{X}_{j} \Gamma_{\mathbb{G}}\right) \circ \iota$ also satisfies the vanishing property on spherical annuli in $\mathbb{G}$. So we are done.

\footnotetext{
${ }^{7}$ We are entitled to interchange the order of integration: indeed, the summands $D^{R}$ with or without an absolute value in the integrand function can be estimated analogously; as for the summand $C^{r, R}$ (which is null without the absolute value in the integrand), if we insert an absolute value in its integrand function, we obtain an integral that can be upper-bounded by $c \log (R / r)<\infty$.
} 
Remark 8.3. Note that the proof of the above point (iii) also exploits the explicit representation that we have for $X_{i} X_{j} \Gamma$ (in terms of the analogous function for the sublaplacian on the lifting Carnot group), and it does not simply follow from growth conditions on the derivatives of $\Gamma$. Also, note that the proof of point (ii) also depends on the estimate on the mixed third order derivatives of $\Gamma$, which required some extra work to be proved, compared to pure derivatives.

\section{Extension to Hörmander OPERATORS With DRIFT}

As we have already announced in Remark 1.4, most of the results in this paper still hold for homogeneous Hörmander operators of the kind

$$
\mathcal{L}=\sum_{i=1}^{m} X_{i}^{2}+X_{0},
$$

i.e., possessing a 'drift' term $X_{0}$. Let us now restate our assumptions in the present context.

Throughout the sequel, we assume that $X=\left\{X_{1}, \ldots, X_{m}, X_{0}\right\}$ is a set of smooth vector fields in $\mathbb{R}^{n}$ satisfying assumptions (H.2)-(H.3), and we replace (H.1) with the following:

(H.1)' There exists a family of dilations of the form (1.1) such that $X_{1}, \ldots, X_{m}$ are $\delta_{\lambda}$-homogeneous of degree 1 , and $X_{0}$ is $\delta_{\lambda}$-homogeneous of degree 2 .

As in the previous sections, we set $q:=\sum_{j=1}^{m} \sigma_{j}$. By assumption (H.3), one has

$$
q>2 \text {. }
$$

Example 9.1. (1). In $\mathbb{R}^{2}$, the operator

$$
X_{1}^{2}+X_{0}=\left(\partial_{x_{1}}\right)^{2}+x_{1}^{k} \partial_{x_{2}} \quad(\text { with } k \in \mathbb{N})
$$

is homogeneous of degree 2 with respect to the dilations $\delta_{\lambda}(x)=\left(\lambda x_{1}, \lambda^{k+2} x_{2}\right)$.

(2). In $\mathbb{R}^{n}$, the operator

$$
X_{1}^{2}+X_{0}=\left(\partial_{x_{1}}\right)^{2}+x_{1} \partial_{x_{2}}+x_{2} \partial_{x_{3}}+\ldots+x_{n-1} \partial_{x_{n}}
$$

is homogeneous of degree 2 with respect to the dilations

$$
\delta_{\lambda}(x)=\left(\lambda x_{1}, \lambda^{3} x_{2}, \lambda^{5} x_{3}, \cdots, \lambda^{2 n-1} x_{n}\right) .
$$

The lifting procedure described in Theorem A can be naturally adapted to the present situation. More precisely, by arguing essentially as in [10, one can prove the following result.

Theorem 9.2. Assume that $X=\left\{X_{0}, X_{1}, \ldots, X_{m}\right\}$ satisfies assumptions (H.1)' and (H.2)-(H.3), of which we inherit the notation. Then the following facts hold:

(1) There exists a graded but not stratified homogeneous group $\mathbb{G}=\left(\mathbb{R}^{N}, *, D_{\lambda}\right)$ (in the sense of [19]) of homogeneous dimension $Q>q$, and there exists a system

$$
\widetilde{X}=\left\{\tilde{X}_{0}, \widetilde{X}_{1}, \ldots, \tilde{X}_{m}\right\}
$$

of Lie-generators for $\operatorname{Lie}(\mathbb{G})$ such that (1.4) in Theorem $A$ holds for every $j=0, \ldots, m$. In particular, $\widetilde{X}_{1}, \ldots, \widetilde{X}_{m}$ are $D_{\lambda}$-homogeneous of degree 1 , while $\widetilde{X}_{0}$ is $D_{\lambda}$-homogeneous of degree 2 .

(2) If $\widetilde{\Gamma}$ is the (unique) smooth fundamental solution of $\sum_{i=1}^{m} \widetilde{X}_{i}^{2}+\widetilde{X}_{0}$ vanishing at infinity constructed in [19], then $\mathcal{L}$ admits a global fundamental solution $\Gamma(x ; y)$ under the form

$$
\Gamma(x ; y):=\int_{\mathbb{R}^{p}} \widetilde{\Gamma}((x, 0) ;(y, \eta)) \mathrm{d} \eta \quad\left(\text { for } x \neq y \text { in } \mathbb{R}^{n}\right) .
$$

Furthermore, if we set $\Gamma_{\mathbb{G}}:=\widetilde{\Gamma}(0 ; \cdot)$, we have

$$
\widetilde{\Gamma}(x, \xi ; y, \eta)=\Gamma_{\mathbb{G}}\left((x, \xi)^{-1} *(y, \eta)\right),
$$

and a formula analogous to (1.7) holds.

(3) $\Gamma$ is smooth out of the diagonal, it is nonnegative but not strictly positive (as in Theorem A); it is locally integrable on $\mathbb{R}^{n} \times \mathbb{R}^{n}$; it vanishes when $x$ or y go to infinity; it is jointly $\delta_{\lambda}$-homogeneous of degree $2-q<0$, that is, (1.8) holds. 
Finally, $\Gamma$ is not symmetric but $\Gamma^{*}(x ; y)=\Gamma(y ; x)$ is the fundamental solution of

$$
\mathcal{L}^{*}=\sum_{i=1}^{m} X_{i}^{2}-X_{0},
$$

and enjoys analogous properties of $\Gamma$.

Remark 9.3. By replacing $X_{0}$ with $Y=-X_{0}$, we see that $\mathcal{L}^{*}$ satisfies properties analogous to $\mathcal{L}$. In particular, if $\widetilde{\Gamma}^{*}$ is the fundamental solution of $\sum_{i=1}^{m} \widetilde{X}_{i}^{2}-\widetilde{X}_{0}$, then the function $\Gamma^{*}$ can be obtained by saturating $\widetilde{\Gamma}^{*}$ as in (1.5). Furthermore, setting $\Gamma_{\mathbb{G}}^{*}=\Gamma^{*}(0 ; \cdot)$, we have

$$
\Gamma_{\mathbb{G}}^{*}\left((x, \xi)^{-1} *(y, \eta)\right)=\widetilde{\Gamma}^{*}(x, \xi ; y, \eta) .
$$

Let us now check how the results about the geometry of vector fields we have reviewed and adapted in Section 2 can be extended to the drift case. First of all, the definition of control distance must be adapted to this situation, as already done in [27]:

$$
d_{X}(x, y):=\inf \{r>0: \text { there exists } \gamma \in C(r) \text { with } \gamma(0)=x \text { and } \gamma(1)=y\},
$$

where $C(r)$ is the set of the absolutely continuous maps $\gamma:[0,1] \rightarrow \mathbb{R}^{n}$ satisfying (a.e. on $[0,1]$ )

$$
\gamma^{\prime}(t)=\sum_{j=0}^{m} a_{j}(t) X_{j}(\gamma(t)), \quad \text { with }\left|a_{0}(t)\right| \leq r^{2} \text { and }\left|a_{j}(t)\right| \leq r \text { for all } j=1, \ldots, m .
$$

We also have to replace the notion of length of a commutator with that of weight of a commutator; this is another fact which is by now standard after [27. For a multi-index

$$
I=\left(i_{1}, \ldots, i_{k}\right), \quad \text { with } i_{1}, \ldots, i_{k} \in\{0,1,2, \ldots, m\},
$$

and a commutator

$$
X_{[I]}:=\left[\left[\left[X_{i_{1}}, X_{i_{2}}\right], X_{i_{3}}\right], \ldots, X_{i_{k}}\right]
$$

we define the weight of $I$ as

$$
|I|=\sum_{j=1}^{k} p_{i_{j}}, \quad \text { where } p_{0}=2 \text { and } p_{i}=1 \text { for } i=1,2, \ldots, m .
$$

With these modifications, all the properties stated in Section 2 still hold with the same statements and the same proofs, as they rely on the theory developed in [27] (which covers also the drift case).

Now, all the properties of $\Gamma$ that we have proved in this paper throughout Sections 4-to- 6 are consequences of the geometric properties stated in Section 2 and of the properties of $\Gamma$ stated in Theorem A, with no reference to the explicit form of the operator $\mathcal{L}$. As a consequence, all the properties proved in Sections 4-to-6 not depending on the symmetry or on the strict positivity of $\Gamma$ (which are the only basic properties of $\Gamma$ not extending to the drift case, see Theorem 9.2) still hold under the assumptions of the present section, with the same proof.

The strict positivity of $\Gamma$ has been used in the proof of the estimates from below on $\Gamma$ (and only to this aim); thus, these estimates do not extend to the the drift case. The symmetry of $\Gamma$ has been used only once, in the proof of Lemma 4.3, formula (4.5). An easy variation of such a proof leads to a slight modification of this identity: by Remark 9.3 , and using the same notation, one has

$$
\Gamma(x, y)=\Gamma^{*}(y, x)=\int_{\mathbb{R}^{p}} \Gamma_{\mathbb{G}}^{*}\left((y, 0)^{-1} *(x, \eta)\right) d \eta
$$

as a consequence,

$$
X_{j_{1}}^{x} \cdots X_{j_{t}}^{x}(\Gamma(\cdot ; y))(x)=\int_{\mathbb{R}^{p}}\left(\widetilde{X}_{j_{1}} \cdots \widetilde{X}_{j_{t}} \Gamma_{\mathbb{G}}^{*}\right)\left((y, 0)^{-1} *(x, \eta)\right) d \eta .
$$

Since the function $\Gamma_{\mathbb{G}}^{*}$ has the same homogeneity and smoothness properties of $\Gamma_{\mathbb{G}}$, the subsequent arguments proceed unchanged, and we have the following theorem. 
Theorem 9.4. Let $\mathcal{L}=\sum_{j=1}^{m} X_{j}^{2}+X_{0}$ satisfy assumptions (H1)'-(H2)-(H3). Moreover, let $\Gamma, \Gamma_{\mathbb{G}}$ and $\Gamma_{\mathbb{G}}^{*}$ be as above. Then the following facts hold.

(I). For any $s, t \geq 1$, and any choice of indexes $i_{1}, \ldots, i_{s}, j_{1}, \ldots, j_{t} \in\{0, \ldots, m\}$, the following representation formulas hold true for $x \neq y$ in $\mathbb{R}^{n}$ :

$$
\begin{aligned}
& X_{i_{1}}^{y} \cdots X_{i_{s}}^{y}(\Gamma(x ; \cdot))(y)=\int_{\mathbb{R}^{p}}\left(\widetilde{X}_{i_{1}} \cdots \widetilde{X}_{i_{s}} \Gamma_{\mathbb{G}}\right)\left((x, 0)^{-1} *(y, \eta)\right) d \eta \\
& X_{j_{1}}^{x} \cdots X_{j_{t}}^{x}(\Gamma(\cdot ; y))(x)=\int_{\mathbb{R}^{p}}\left(\widetilde{X}_{j_{1}} \cdots \widetilde{X}_{j_{t}} \Gamma_{\mathbb{G}}^{*}\right)\left((y, 0)^{-1} *(x, \eta)\right) d \eta \\
& X_{j_{1}}^{x} \cdots X_{j_{t}}^{x} X_{i_{1}}^{y} \cdots X_{i_{s}}^{y} \Gamma(x ; y) \\
& \quad=\int_{\mathbb{R}^{p}}\left(\widetilde{X}_{j_{1}} \cdots \widetilde{X}_{j_{t}}\left(\left(\widetilde{X}_{i_{1}} \cdots \widetilde{X}_{i_{s}} \Gamma_{\mathbb{G}}\right) \circ \iota\right)\right)\left((y, 0)^{-1} *(x, \eta)\right) d \eta
\end{aligned}
$$

Here $\iota$ denotes the inversion map of the Lie group $\mathbb{G}$.

(II). Given any $Z_{1}, \ldots, Z_{r} \in\left\{X_{0}^{x}, \ldots, X_{m}^{x}, X_{0}^{y}, \ldots, X_{m}^{y}\right\}$, we define

$$
\begin{aligned}
& z:=Z_{1} \cdots Z_{r} ; \\
& |z|:=\sum_{k=1}^{r}\left|Z_{k}\right|, \quad \text { where }\left|Z_{k}\right|:= \begin{cases}2, & \text { if } Z \in\left\{X_{0}^{x}, X_{0}^{y}\right\}, \\
1, & \text { otherwise }\end{cases}
\end{aligned}
$$

Then, there exists a constant $C>0$

$$
|z \Gamma(x ; y)| \leq C \frac{d_{X}(x, y)^{2-|z|}}{\left|B_{X}\left(x, d_{X}(x, y)\right)\right|}
$$

for any $x \neq y \in \mathbb{R}^{n}$. In particular, for every fixed pole $x \in \mathbb{R}^{n}$ we have

$$
\lim _{|y| \rightarrow \infty} z \Gamma(x ; y)=0 .
$$

(III). Suppose that $n>2$. Then one has

$$
0 \leq \Gamma(x ; y) \leq C \frac{d_{X}(x, y)^{2}}{\left|B_{X}\left(x, d_{X}(x, y)\right)\right|},
$$

for any $x, y \in \mathbb{R}^{n}($ with $x \neq y)$. Here $C \geq 1$ is a structural constant.

(IV). Suppose that $n=2$. For every compact set $K \subseteq \mathbb{R}^{n}$ there exist a structural constant $c>0$ and a real number $R>0$ (all depending on $K$ ) such that

$$
0 \leq \Gamma(x ; y) \leq c \frac{d_{X}(x, y)^{2}}{\left|B_{X}\left(x, d_{X}(x, y)\right)\right|} \cdot \log \left(\frac{R}{d_{X}(x, y)}\right),
$$

uniformly for $x \neq y$ in $K$. Moreover, for every fixed pole $x \in \mathbb{R}^{n}$, there exists a constant $\gamma(x)>0$ and $0<\varepsilon(x)<1$ such that

$$
\Gamma(x ; y) \leq \gamma(x) F(x, y)
$$

for any $y$ such that $0<d_{X}(x, y)<\varepsilon(x)$. Here, $F(x, y)$ is as in Theorem 1.3 .

\section{REFERENCES}

[1] B. Abbondanza, A. Bonfiglioli: On the Dirichlet problem and the inverse mean value theorem for a class of divergence form operators. J. London Math. Soc. 87 (2013), 321-34.

[2] E. Battaglia, A. Bonfiglioli: Normal families of functions for subelliptic operators and the theorems of Montel and Koebe. J. Math. Anal. Appl. 409 (2014), 1-12.

[3] E. Battaglia, S. Biagi, A. Bonfiglioli: The strong maximum principle and the Harnack inequality for a class of hypoelliptic non-Hörmander operators. Ann. Inst. Fourier (Grenoble) 66 (2016), 589-631.

[4] E. Battaglia, A. Bonfiglioli: An invariant Harnack inequality for a class of subelliptic operators under global doubling and Poincaré assumptions, and applications. J. Math. Anal. Appl. 460 (2018), 302-320.

[5] W. Bauer, K. Furutani, C. Iwasaki: Fundamental solution of a higher step Grushin type operator. Adv. Math. 271 (2015), 188-234. 
[6] R. Beals, B. Gaveau, P. Greiner: On a geometric formula for the fundamental solution of subelliptic Laplacians. Math. Nachr. 181 (1996), 81-163.

[7] R. Beals, B. Gaveau, P. Greiner: Uniform hypoelliptic fundamental solutions. J. Math. Pures Appl. (9) 77 (1998), 209-248.

[8] R. Beals, B. Gaveau, P. Greiner, Y. Kannai: Transversally elliptic operators. Bull. Sci. Math. 128 (2004), $531-576$.

[9] S. Biagi, A. Bonfiglioli: A completeness result for time-dependent vector fields and applications. Commun. Contemp. Math. 17 (2015), 1-26.

[10] S. Biagi, A. Bonfiglioli: The existence of a global fundamental solution for homogeneous Hörmander operators via a global lifting method. Proc. Lond. Math. Soc. 114 (2017), 855-889.

[11] S. Biagi, A. Bonfiglioli: "An Introduction to the Geometrical Analysis of Vector Fields - with Applications to Maximum Principles and Lie Groups", World Scientific Publishing, Singapore (2019).

[12] A. Bonfiglioli, E. Lanconelli: Subharmonic functions in sub-Riemannian settings. J. Eur. Math. Soc. 15 (2013), $387-441$.

[13] A. Bonfiglioli, E. Lanconelli, F. Uguzzoni: "Stratified Lie Groups and Potential Theory for their sub-Laplacians", Springer Monographs in Mathematics 26, Springer, New York, N.Y., 2007.

[14] J.-M. Bony: Principe du maximum, inégalité de Harnack et unicité du problème de Cauchy pour les opérateurs elliptiques dégénérés. Ann. Inst. Fourier (Grenoble) 19 (1969), 277-304.

[15] M. Bramanti, L. Brandolini: Hörmander operators. Submitted book.

[16] M. Bramanti, L. Brandolini, M. Manfredini, M. Pedroni: Fundamental solutions and local solvability for nonsmooth Hörmander's operators. Mem. Amer. Math. Soc. 249 (2017), no. 1182.

[17] R. R. Coifman, G. Weiss: Analyse harmonique non-commutative sur certains espaces homogènes. Étude de certaines intégrales singulières. Lecture Notes in Mathematics, 242. Springer-Verlag, Berlin-New York, 1971.

[18] M. Derridj: Un problème aux limites pour une classe d'opérateurs du second ordre hypoelliptiques. Ann. Inst. Fourier (Grenoble) 21 (1971), 99-148.

[19] G.B. Folland: Subelliptic estimates and function spaces on nilpotent Lie groups. Ark. Mat. 13 (1975), 161-207.

[20] G.B. Folland: On the Rothschild-Stein lifting theorem, Comm. Partial Differential Equations 2 (1977), 161-207.

[21] B. Franchi, E. Lanconelli: Une métrique associée à une classe d'opérateurs elliptiques dégénérés. In: Conference on Linear Partial and Pseudodifferential Operators, Torino, 1982, in: Rend. Semin. Mat. Univ. Politec. Torino, 1983, 1984, pp. 105-114 (special issue).

[22] P.C. Greiner: A fundamental solution for a nonelliptic partial differential operator. Can. J. Math. 31 (1979), $1107-1120$.

[23] P. Hajłasz, P. Koskela: Sobolev met Poincaré. Mem. Amer. Math. Soc. 145 (2000).

[24] L. Hörmander: Hypoelliptic second order differential equations. Acta Math. 119 (1967), 147-171.

[25] D. Jerison: The Poincaré inequality for vector fields satisfying Hörmander's condition, Duke Math. J. 53 (1986), 503-523.

[26] A. Kogoj, E. Lanconelli: On semilinear $\Delta_{\lambda}$-Laplace equation. Nonlinear Anal. 75 (2012), 4637-4649.

[27] A. Nagel, E. M. Stein, S. Wainger: Balls and metrics defined by vector fields I: Basic properties. Acta Mathematica, 155 (1985), 130-147.

[28] L.P. Rothschild, E.M. Stein: Hypoelliptic differential operators and nilpotent groups, Acta Math. 137 (1976), $247-320$.

[29] A. Sánchez-Calle: Fundamental solutions and geometry of the sum of squares of vector fields. Invent. Math., 78 (1984), 143-160.

Stefano Biagi: Dipartimento di Matematica, Politecnico di Milano, Via Bonardi 9, I-20133 Milano, ITALY.

E-mail address: stefano.biagi@polimi.it

Andrea Bonfiglioli: Dipartimento di Matematica, Alma Mater Studiorum - Università di Bologna, Piazza Porta San Donato 5, I-40126 Bologna, Italy.

E-mail address: andrea.bonfiglioli6@unibo.it

Marco Bramanti: Dipartimento di Matematica, Politecnico di Milano, Via Bonardi 9, I-20133 Milano, ITALY.

E-mail address: marco.bramanti@polimi.it 